\title{
Mechanistic Studies on the Generation and Properties of Super-electrophilic Singlet Carbenes from Bis(perfluoroalkanesulfonyl)bromonium Ylides
}

Kazunori Miyamoto, ${ }^{*}, \dagger$ Susumu Iwasaki, ${ }^{\S}$ Ryusuke Doi,${ }^{\S}$ Taiga Ota,${ }^{\S}$ Yufuko Kawano, ${ }^{\S}$ Junpei Yamashita, ${ }^{\S}$ Yuuta Sakai, ${ }^{\S}$ Norihiro Tada,${ }^{\perp}$ Masahito Ochiai, ${ }^{\S}$ Satoko Hayashi, ${ }^{\text {ॠ Waro }}$

$$
\text { Nakanishi, }{ }^{*, \Uparrow} \text { and Masanobu Uchiyama*,†, }
$$

Graduate School of Pharmaceutical Sciences, The University of Tokyo, 7-3-1 Hongo Bunkyo-ku,

$$
\text { Tokyo 113-0033 (Japan) }
$$

Email: kmiya@mol.f.u-tokyo.ac.jp

\section{Table of Contents}

1. UV absorption spectra 2

2. Eyring plot 3

3. Reaction of ylide 9a and cis-4-octene in the presence of 9,10-dihydroanthracene 4

4. Synthesis of authentic samples by Billard anti-1,2-S, $O$-addition and triflination sequence 5

5. Calculations $6-15$

6. Spectra 16-51 
1. Thermal decomposition of bromonium ylide 9a measured spectrophotometrically in perfluorodecalin in the presence and absence of 1-octene.

A
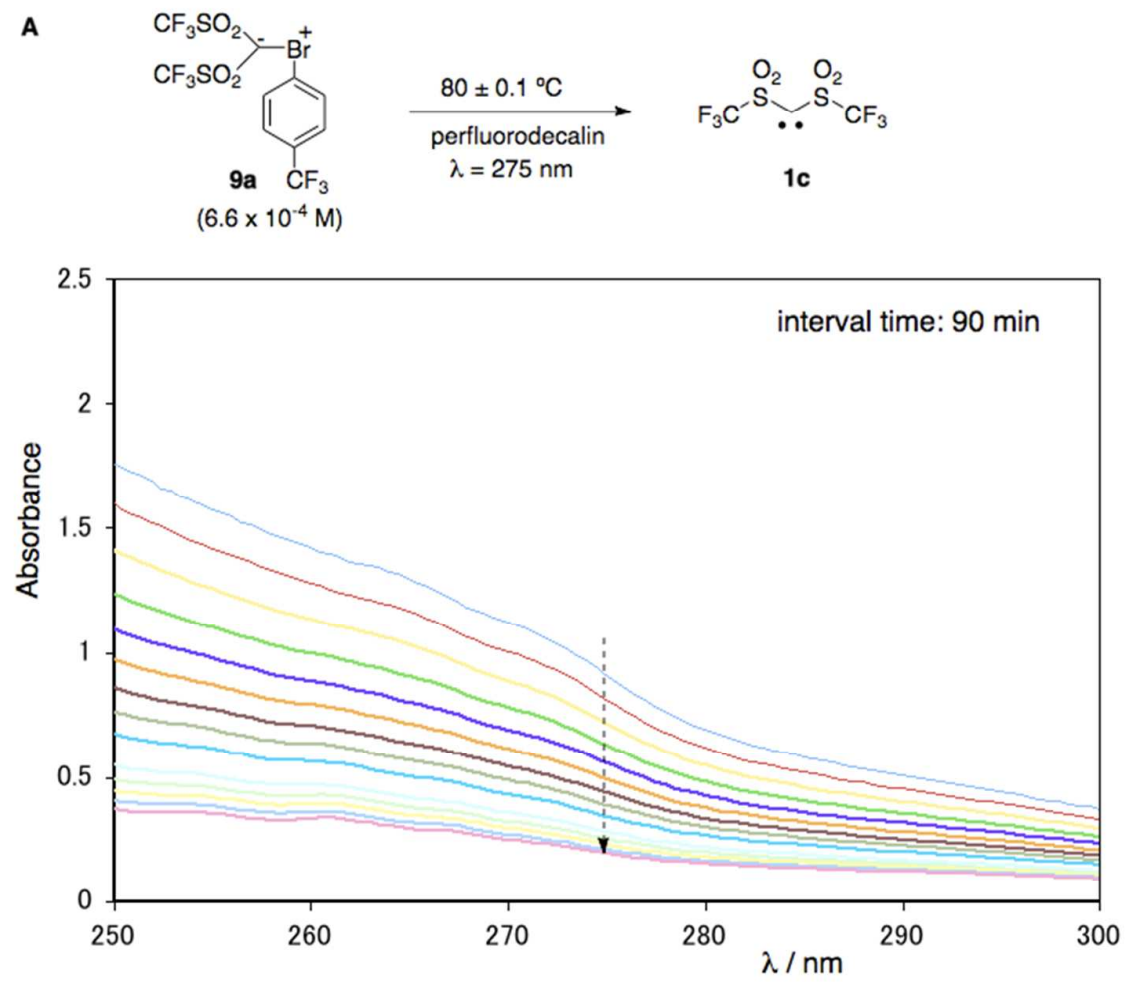

B
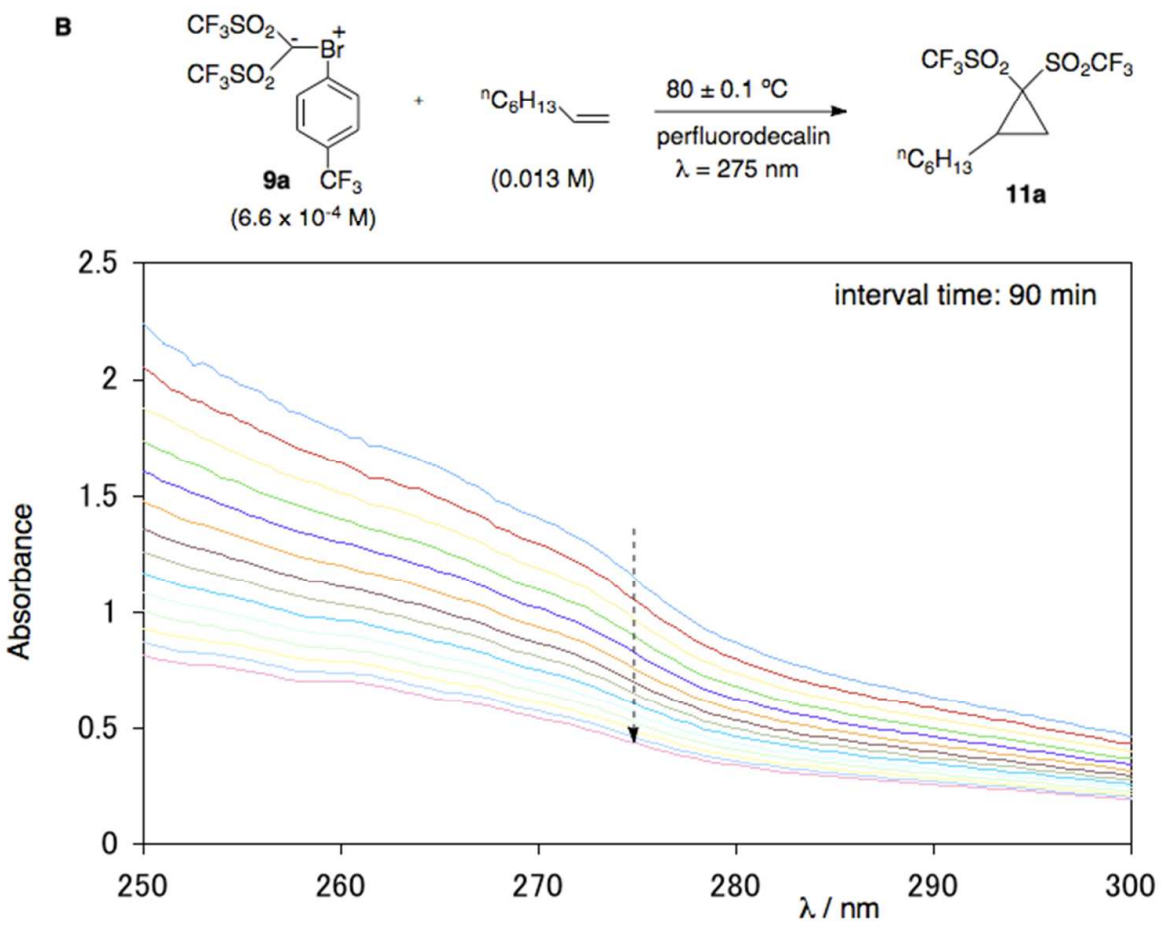

Figure S1. Time-dependent UV absorbance change: (A) the thermal decomposition of bromonium ylide 1a in perfluorodecalin as a solvent, (B) the reaction of bromonium ylide 9a with 1-octene in perfluorodecalin as a solvent. Initial concentration of ylide $9 \mathrm{a}, 6.6 \times 10^{-4} \mathrm{M}$. 
2. Eyring plot
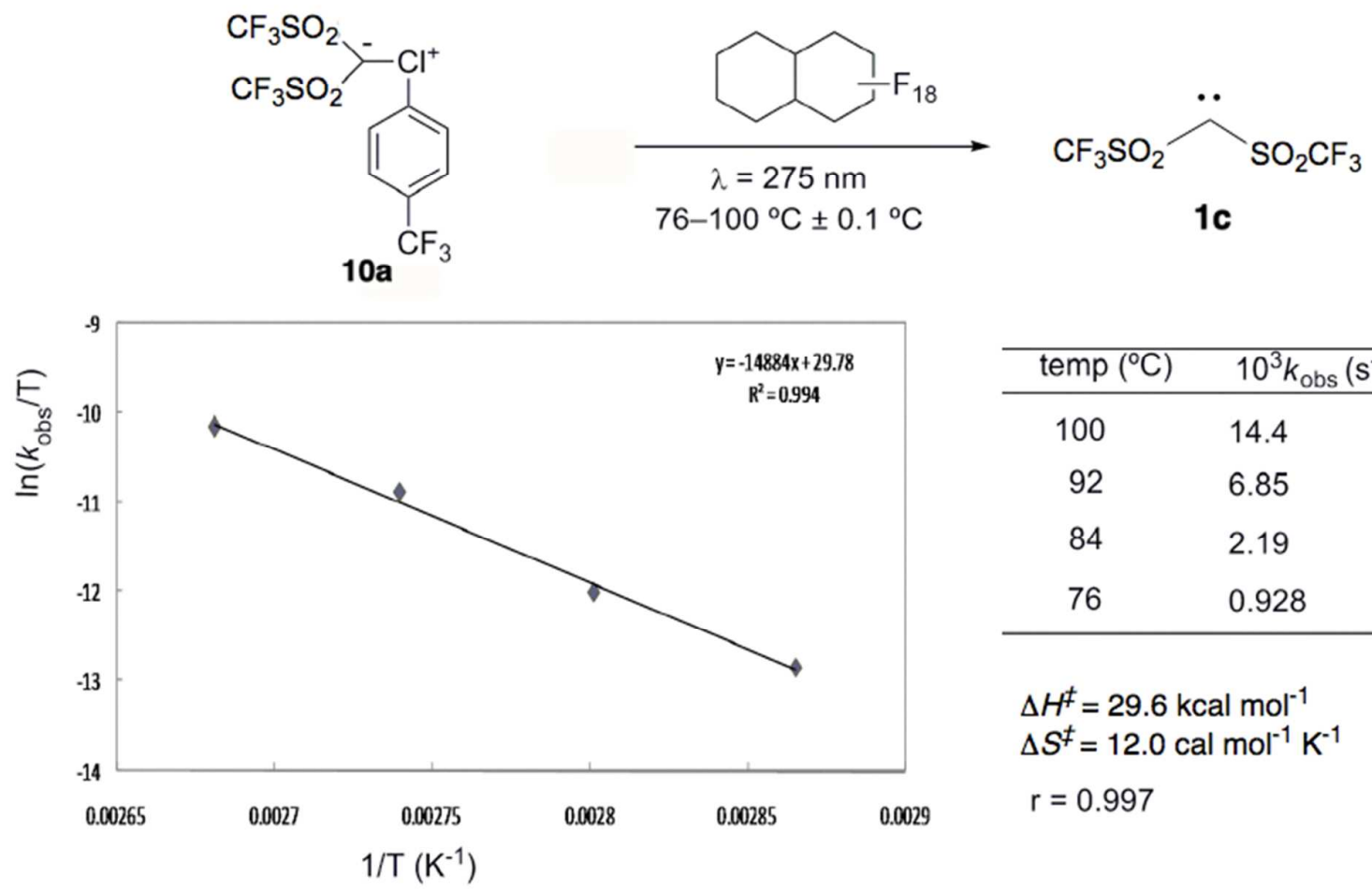

\begin{tabular}{cl}
\hline temp $\left({ }^{\circ} \mathrm{C}\right)$ & $10^{3} k_{\text {obs }}\left(\mathrm{s}^{-1}\right)$ \\
\hline 100 & 14.4 \\
92 & 6.85 \\
84 & 2.19 \\
76 & 0.928 \\
\hline
\end{tabular}

$\Delta H^{\ddagger}=29.6 \mathrm{kcal} \mathrm{mol}^{-1}$ $\Delta S^{\ddagger}=12.0 \mathrm{cal} \mathrm{mol}^{-1} \mathrm{~K}^{-1}$ $r=0.997$

Figure S2. Activation parameter for thermal decomposition of chloronium ylide 10a in perfluorodecalin. 


\section{Reaction of ylide 9a and cis-4-octene in the presence of 9,10-dihydroanthracene}

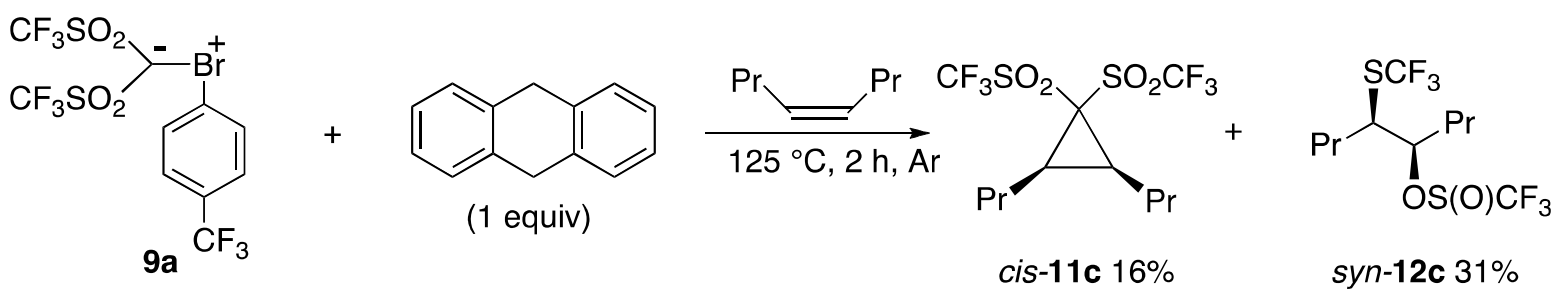

Scheme $\mathbf{S 1}$ Thermal solvolysis of bromonium ylide $\mathbf{9 a}$ in cis-4-octene in the presence of 9,10-dihydroanthracene (1 equiv). Careful ${ }^{1} \mathrm{H}$ NMR analysis of the crude reaction mixture failed to detect formation of the stereoisomeric trans-11c under the conditions. After 2 hours, almost all 9,10-dihydroanthracene (93\%) was recovered unchanged. A comparable result (cis-11c: $25 \%$ ) was obtained in the thermal solvolysis of $\mathbf{9 a}$ in cis-4-octene under air condition. 


\section{Synthesis of authentic samples by Billard anti-1,2-S,O-addition and triflination sequence}

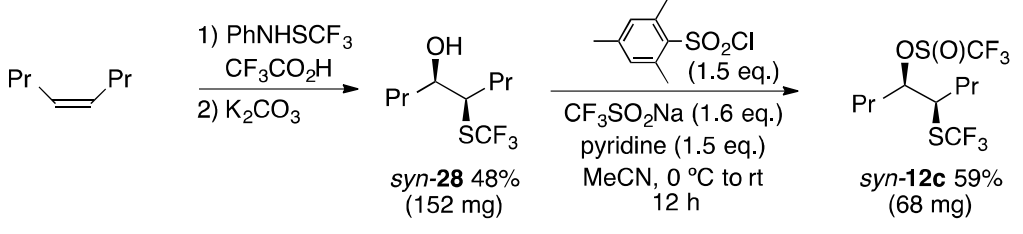

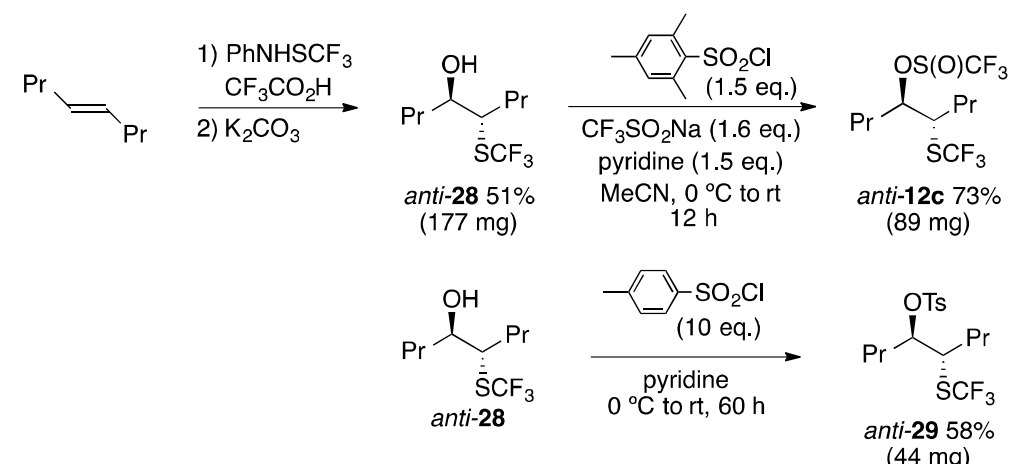

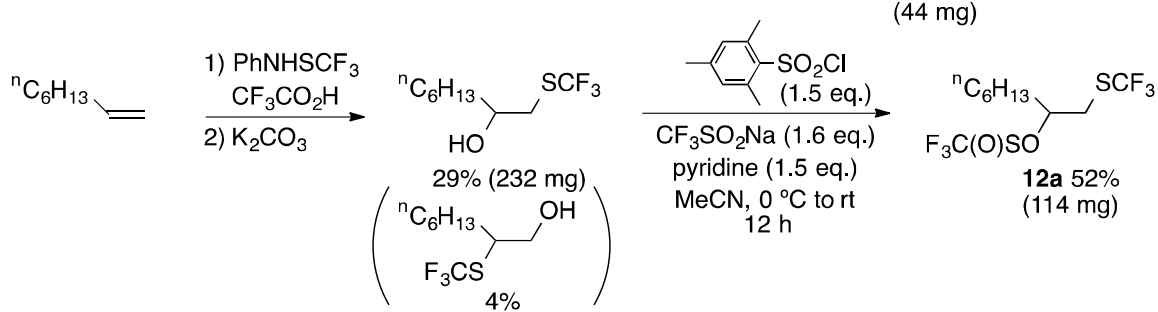

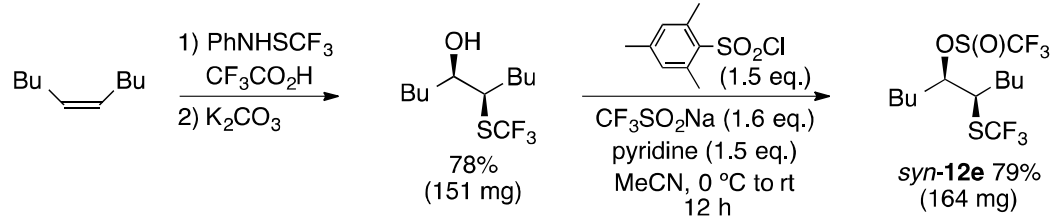

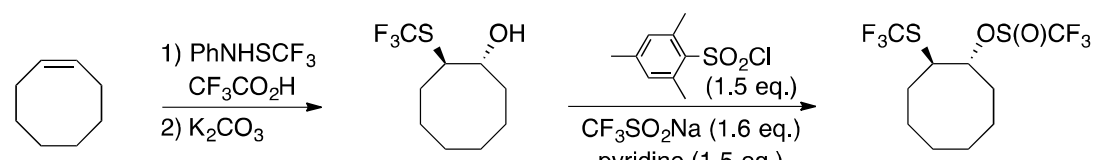

$$
\begin{aligned}
& 15 \% \text { pyridine (1.5 eq.) trans-12g } 21 \% \\
& (25 \mathrm{mg}) \quad 12 \mathrm{~h} \quad(8 \mathrm{mg}) \\
& \Rightarrow \underset{2 \mathrm{~K}_{2} \mathrm{CO}_{3}}{\mathrm{CF}_{3} \mathrm{CO}_{2} \mathrm{H}} \\
& 58 \% \text { pyridine (1.5 eq.) trans- } 12 \mathrm{f} 51 \% \\
& \begin{array}{c}
\text { (110 mg) } \quad \mathrm{MeCN}, 0^{\circ} \mathrm{C} \text { to rt } \\
12 \mathrm{~h}
\end{array} \\
& \text { 1) } \underset{2 \mathrm{KhNHSCF}_{3} \mathrm{KO}_{3}}{\stackrel{\mathrm{CF}_{3} \mathrm{CO}_{2} \mathrm{H}}{2 \mathrm{CO}_{3}}} \frac{\mathrm{OH}(\mathrm{O}) \mathrm{CF}_{3}}{\mathrm{CF}_{3} \mathrm{SO}_{2} \mathrm{Na}(1.6 \mathrm{eq} .)} \\
& \begin{array}{ccc}
40 \% & \mathrm{MeCN}, 0^{\circ} \mathrm{C} \text { to rt } & \text { trans-12h } 75 \% \\
(150 \mathrm{mg}) & 12 \mathrm{~h} & (155 \mathrm{mg})
\end{array}
\end{aligned}
$$

Scheme S1 Synthesis of authentic samples of $\beta$-trifluoromethylthio trifluoromethylsulfinates by two-step sequences. The transient species 28 was converted into know tosylate $29 .{ }^{1} \mathrm{H} /{ }^{19} \mathrm{~F}$ NMR chemical shifts of compound 29 were in good agreement with Billard's reported data (see, Ferry, A.; Billard, T.; Langlois, B. R.; Bacque, E. Angew. Chem. Int. Ed. 2009, 48, 8551). 


\section{Calculations}

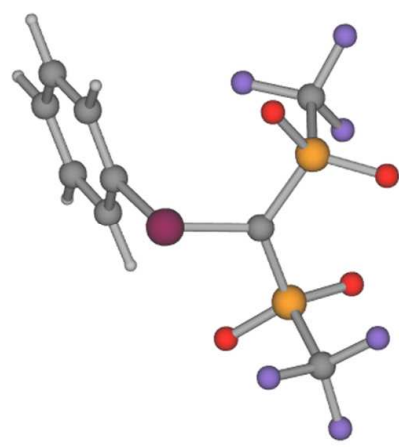

1c

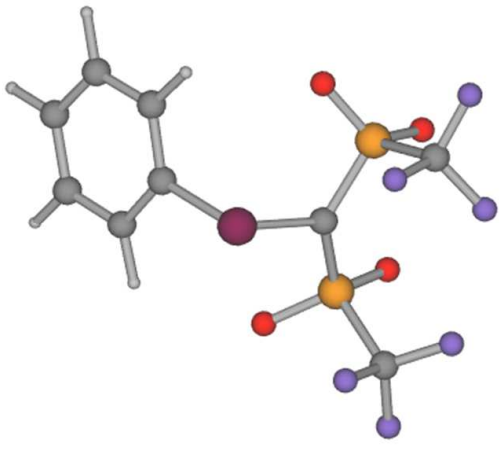

1c-cis-a

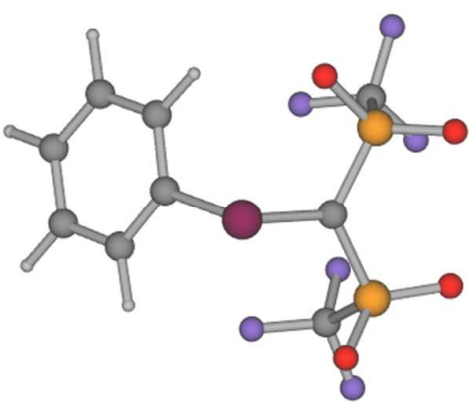

1c-cis-b

Table S1. Relative energies: 9c

\begin{tabular}{lcl}
\hline Compd & $\Delta E / \mathrm{kcal} \mathrm{mol}^{-1}$ & Form \\
\hline 9c & $0.0^{a}$ & trans \\
9c-cis- $a$ & 1.3 & cis- $a$ \\
9c-cis- $b$ & 3.2 & cis- $b$ \\
\hline
\end{tabular}

${ }^{a} E=-4611.1058$ au.

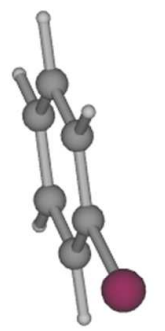

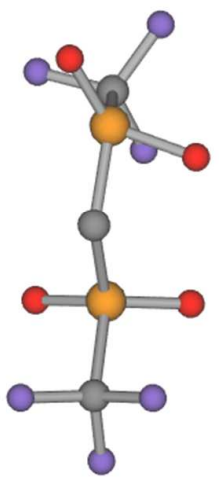

9c (TS)-cis
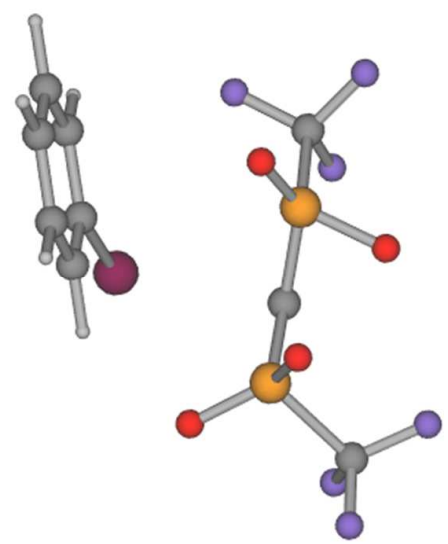

9c (TS)-trans

Table S2. Relative energies: 9c (TS)

\begin{tabular}{lcc}
\hline Compd & $\Delta E / \mathrm{kcal} \mathrm{mol}^{-1}$ & Form \\
\hline $\mathbf{9 c}$ (TS)-cis & $0.0^{a}$ & cis \\
$\mathbf{9 c}$ (TS)-trans & 2.4 & trans \\
\hline
\end{tabular}

${ }^{a} E=-4611.0519$ au. 
Figure S3. Energy profile of ${ }^{1} 1 \mathrm{c}$ and ${ }^{3} 1 \mathrm{c}$.

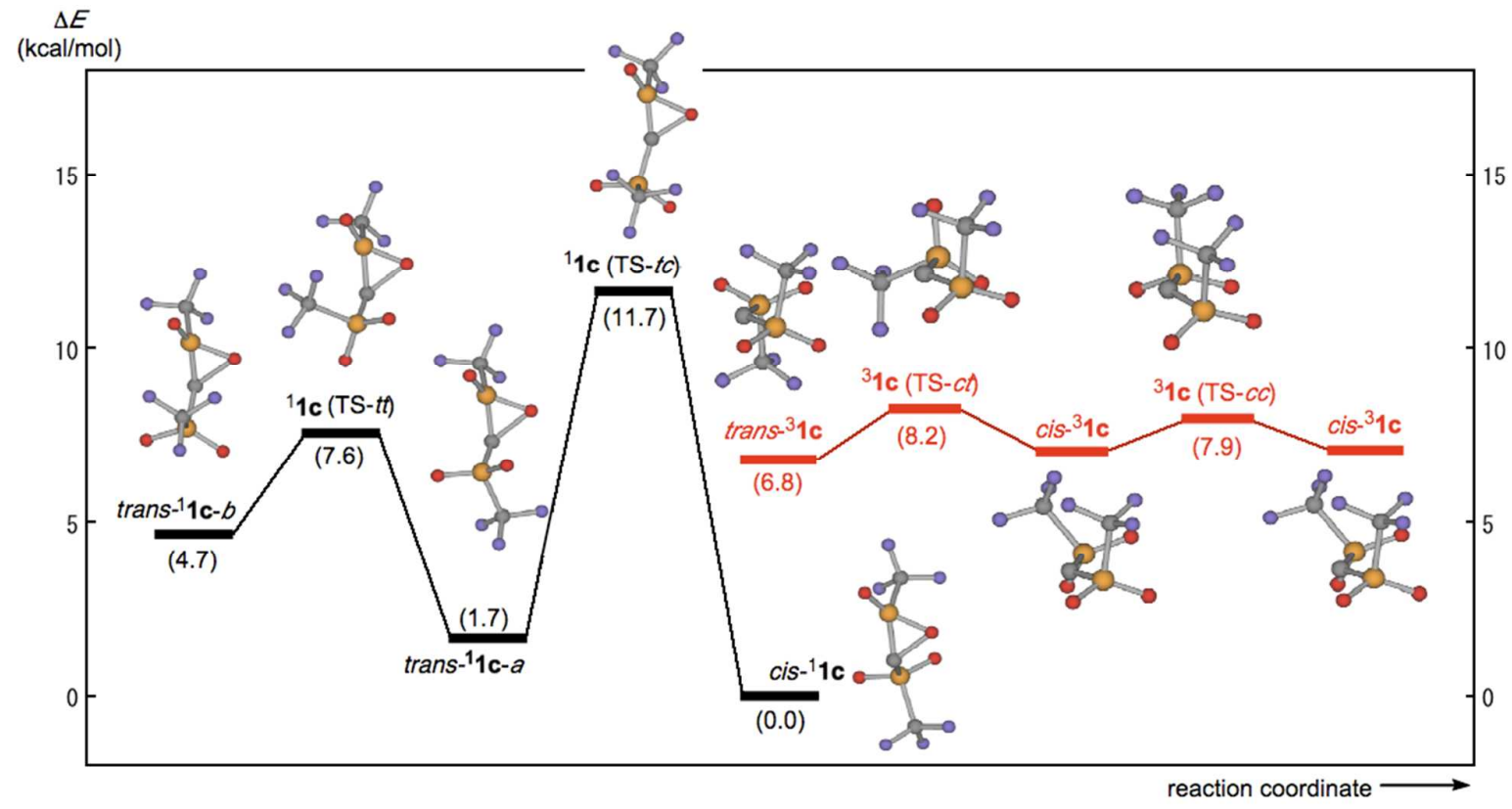

Table S3. Relative energies of ${ }^{1} \mathbf{1 c}$

\begin{tabular}{lcc}
\hline Compound & $\Delta E / \mathrm{kcal} \mathrm{mol}^{-1}$ & Form \\
\hline cis- $^{1} \mathbf{1 c}$ & $0.0^{a}$ & cis \\
trans $^{-1} \mathbf{1} \mathbf{c}-a$ & 1.7 & trans- $a$ \\
trans $^{-1} \mathbf{1}-\mathrm{c}-b$ & 4.7 & trans- $b$ \\
${ }^{1} \mathbf{1 c}(\mathrm{TS}-\mathrm{t} t)$ & 7.6 & trans-to-trans \\
${ }^{1} \mathbf{1} \mathbf{c}(\mathrm{TS}-\mathrm{c} t)$ & 11.7 & cis-to-trans \\
\hline
\end{tabular}

${ }^{a} E=-1807.4033$ au.

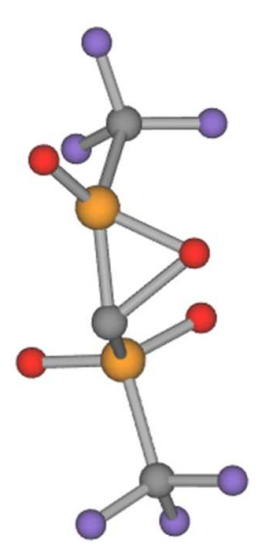

${ }^{\text {cis }}{ }^{1}{ }^{1} \mathbf{c}$

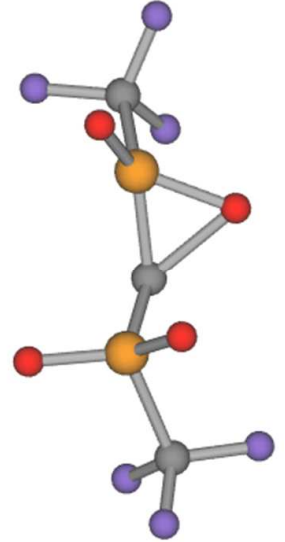

trans $-{ }^{1} \mathbf{1}-a$

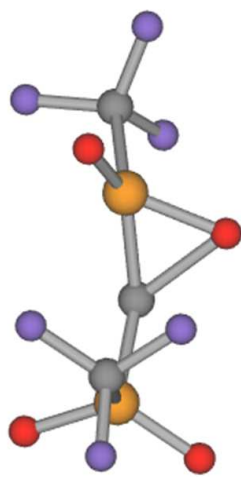

trans- ${ }^{1} \mathbf{1} \mathbf{c}-b$

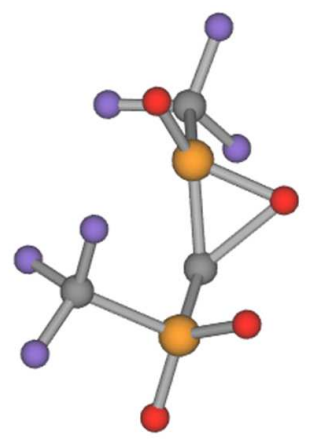

${ }^{1} 1 \mathrm{c}(\mathrm{TS}-t t)$

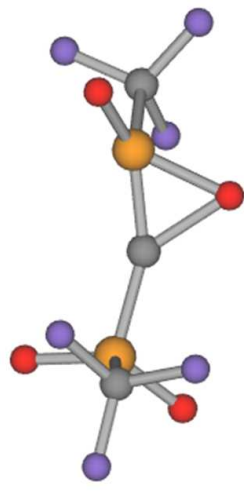

${ }^{1} \mathbf{1 c}(\mathrm{TS}-c t)-a$

Table S4. Relative energies of ${ }^{3} \mathbf{1 c}$

\begin{tabular}{lcc}
\hline Compound & $\Delta E / \mathrm{kcal} \mathrm{mol}^{-1}$ & Form \\
\hline${ }_{\text {trans- }}{ }^{3} \mathbf{1 c}$ & $0.0^{a}$ & trans \\
cis- $^{-} \mathbf{c}$ & 0.3 & cis \\
${ }^{3} \mathbf{1}$ (TS-cc) & 1.1 & cis-to-cis \\
${ }^{3} \mathbf{1 c}($ TS-ct $)$ & 1.4 & cis-to-trans \\
\hline
\end{tabular}


${ }^{a} E=-1807.3924$ au.

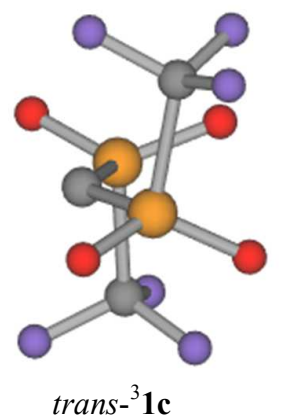

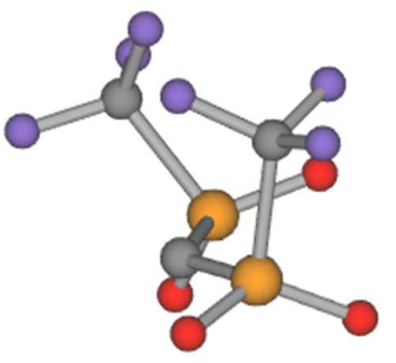

cis- $^{3} \mathbf{1 c}$

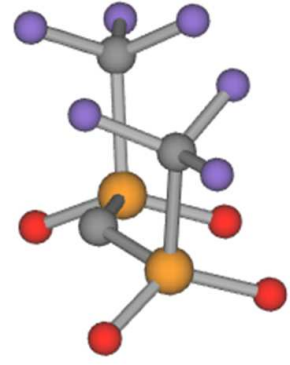

${ }^{3} 1 \mathrm{c}(\mathrm{TS}-c c)$

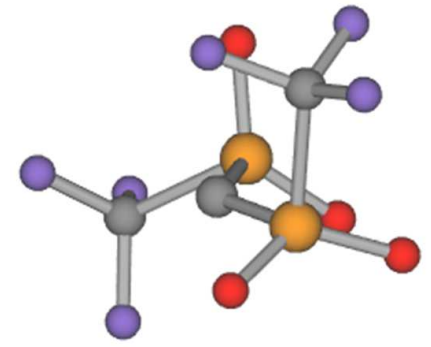

${ }^{3} \mathbf{1 c}(\mathrm{TS}-c t)$

Optimized structures given by Cartesian coordinates for examined molecules, together with the total energies

1) Figure 4

\begin{tabular}{lccc} 
Bromonium ylide 9c & \multicolumn{3}{l}{} \\
MP2 & -4611.1057956 & au & \\
C1 & -0.58036 & 0.06488 & -0.30102 \\
S2 & -1.61217 & -0.57330 & 0.92404 \\
O3 & -1.12864 & -1.90288 & 1.27769 \\
O4 & -1.90721 & 0.44641 & 1.90725 \\
C5 & -3.21690 & -0.90443 & 0.03672 \\
F6 & -4.06299 & -1.50358 & 0.86824 \\
F7 & -2.98516 & -1.71603 & -1.00381 \\
F8 & -3.75257 & 0.22160 & -0.40600 \\
S9 & -0.40635 & 1.73792 & -0.69393 \\
O10 & 0.32065 & 1.81896 & -1.95263 \\
O11 & -1.64170 & 2.44562 & -0.43728 \\
C12 & 0.81016 & 2.39250 & 0.56192 \\
F13 & 1.09810 & 3.65629 & 0.26281 \\
F14 & 1.93728 & 1.67066 & 0.50844 \\
F15 & 0.31982 & 2.32988 & 1.78919 \\
Br16 & 0.42963 & -1.15026 & -1.27067 \\
C17 & 2.11153 & -1.25213 & -0.33938 \\
C18 & 3.21738 & -0.68429 & -0.96856 \\
C19 & 2.13024 & -1.83485 & 0.92606 \\
C20 & 4.43221 & -0.71951 & -0.27336 \\
C21 & 3.35914 & -1.84442 & 1.59572 \\
C22 & 4.50084 & -1.29183 & 1.00158 \\
H23 & 3.13219 & -0.21113 & -1.94198 \\
H24 & 1.22668 & -2.24270 & 1.36925 \\
H25 & 5.31659 & -0.28162 & -0.72643 \\
H26 & 3.41750 & -2.28499 & 2.58655 \\
H27 & 5.44582 & -1.30413 & 1.53605
\end{tabular}


26 (TS)

MP2 = -4611.0519176 au

$\begin{array}{lccc}\text { C1 } & -1.09914 & 0.44553 & -0.94492 \\ \text { S2 } & -1.85060 & 0.35449 & 0.71816 \\ \text { O3 } & -1.00414 & -0.39079 & 1.63633 \\ \text { O4 } & -2.47431 & 1.61363 & 1.06584 \\ \text { C5 } & -3.26731 & -0.77638 & 0.26846 \\ \text { F6 } & -3.97400 & -0.97594 & 1.37651 \\ \text { F7 } & -2.82820 & -1.94523 & -0.18457 \\ \text { F8 } & -4.03455 & -0.20519 & -0.64769 \\ \text { S9 } & 0.19251 & 1.52413 & -1.12746 \\ \text { O10 } & 1.22599 & 1.30986 & -2.10997 \\ \text { O11 } & -1.01504 & 2.40829 & -1.30236 \\ \text { C12 } & 1.09480 & 2.25494 & 0.35443 \\ \text { F13 } & 1.57718 & 3.43027 & -0.02990 \\ \text { F14 } & 2.08723 & 1.45567 & 0.70119 \\ \text { F15 } & 0.26529 & 2.42217 & 1.36600 \\ \text { Br16 } & 0.25750 & -1.93603 & -1.02107 \\ \text { C17 } & 1.85336 & -1.55138 & -0.06728 \\ \text { C18 } & 2.97789 & -1.13398 & -0.78570 \\ \text { C19 } & 1.86143 & -1.68639 & 1.32416 \\ \text { C20 } & 4.14691 & -0.83736 & -0.07612 \\ \text { C21 } & 3.04070 & -1.37913 & 2.01148 \\ \text { C22 } & 4.18005 & -0.95449 & 1.31783 \\ \text { H23 } & 2.93524 & -1.03443 & -1.86534 \\ \text { H24 } & 0.96920 & -2.00750 & 1.85014 \\ \text { H25 } & 5.02941 & -0.51144 & -0.61942 \\ \text { H26 } & 3.06351 & -1.47514 & 3.09333 \\ \text { H27 } & 5.09080 & -0.72017 & 1.86105\end{array}$

\section{7 (MS)}

$\begin{array}{lccc}\text { MP2 } & -4611.0614297 & & \\ \text { C1 } & -1.37060 & 0.62253 & -1.03029 \\ \text { S2 } & -2.00548 & 0.46448 & 0.66336 \\ \text { O3 } & -1.05120 & -0.24293 & 1.50466 \\ \text { O4 } & -2.66923 & 1.68993 & 1.06250 \\ \text { C5 } & -3.35755 & -0.76477 & 0.28683 \\ \text { F6 } & -3.99939 & -1.01502 & 1.42395 \\ \text { F7 } & -2.84534 & -1.89207 & -0.18642 \\ \text { F8 } & -4.20643 & -0.25435 & -0.59640 \\ \text { S9 } & 0.20083 & 1.23100 & -1.19516 \\ \text { O10 } & 1.16091 & 0.95559 & -2.23650 \\ \text { O11 } & -1.12065 & 2.14225 & -1.38934 \\ \text { C12 } & 1.02716 & 2.35420 & 0.08743 \\ \text { F13 } & 1.23054 & 3.52365 & -0.49507 \\ \text { F14 } & 2.17289 & 1.81321 & 0.43301 \\ \text { F15 } & 0.23212 & 2.48763 & 1.12321\end{array}$




$\begin{array}{lccc}\text { Br16 } & 0.59621 & -2.35277 & -0.83662 \\ \text { C17 } & 1.99492 & -1.49649 & 0.11784 \\ \text { C18 } & 3.14687 & -1.09951 & -0.57133 \\ \text { C19 } & 1.87092 & -1.31703 & 1.50047 \\ \text { C20 } & 4.19409 & -0.51035 & 0.14632 \\ \text { C21 } & 2.92899 & -0.72416 & 2.19945 \\ \text { C22 } & 4.09070 & -0.32397 & 1.52947 \\ \text { H23 } & 3.22027 & -1.25108 & -1.64365 \\ \text { H24 } & 0.96997 & -1.63586 & 2.01248 \\ \text { H25 } & 5.09381 & -0.20528 & -0.38113 \\ \text { H26 } & 2.84155 & -0.58519 & 3.27355 \\ \text { H27 } & 4.91011 & 0.12793 & 2.08098\end{array}$

${ }^{1} \mathbf{1 c}\left(=\right.$ cis $\left.^{-1} \mathbf{1} \mathbf{c}+\mathrm{PhBr}\right)$

MP2 $=-4611.040532 \mathrm{au}$

$\begin{array}{llll}\text { C1 } & -0.20539 & 1.13270 & -0.14475\end{array}$

S2 $\quad-1.04576 \quad-0.46140 \quad-0.28809$

O3 $\quad-0.68164 \quad-1.38330 \quad 0.77360$

O4 $-1.02437 \quad-0.81409 \quad-1.69685$

$\begin{array}{llll}\text { C5 } & -2.77069 & 0.12872 & 0.11064\end{array}$

$\begin{array}{llll}\text { F6 } & -3.57192 & -0.92976 & 0.10913\end{array}$

$\begin{array}{llll}\text { F7 } & -2.77793 & 0.69103 & 1.31448\end{array}$

$\begin{array}{llll}\text { F8 } & -3.18742 & 1.00289 & -0.79207\end{array}$

$\begin{array}{llll}\text { S9 } & 1.48226 & 1.01366 & -0.14017\end{array}$

$\begin{array}{llll}\text { O10 } & 2.41614 & 1.96701 & -0.68392\end{array}$

$\begin{array}{llll}\text { O11 } & 0.70932 & 1.17193 & 1.22754\end{array}$

$\begin{array}{llll}\mathrm{C} 12 & 2.41286 & -0.64759 & 0.10529\end{array}$

F13 $3.68854 \quad-0.39763 \quad-0.11262$

$\begin{array}{llll}\text { F14 } & 1.95827 & -1.50338 & -0.78970\end{array}$

$\begin{array}{llll}\text { F15 } & 2.22822 & -1.09112 & 1.32213\end{array}$

${ }^{3} \mathbf{1 c}\left(=\right.$ trans $\left.^{-3} \mathbf{1 c}\right)+\mathrm{PhBr}$

MP2 $=-4611.029693 \mathrm{au}$

$\begin{array}{llll}\mathrm{C} 1 & 0.00000 & 0.00000 & 0.83135\end{array}$

$\begin{array}{llll}\mathrm{S} 2 & 0.89077 & 1.37046 & 0.12451\end{array}$

$\begin{array}{llll}\mathrm{O} 3 & 1.76089 & 1.88578 & 1.15481\end{array}$

O4 $\quad 1.33562 \quad 0.98872 \quad-1.19712$

$\begin{array}{llll}\text { C5 } & -0.50816 & 2.59559 & -0.08694\end{array}$

$\begin{array}{llll}\text { F6 } & 0.00000 & 3.71132 & -0.58361\end{array}$

F7 $\quad-1.06594 \quad 2.83570 \quad 1.09348$

F8 $\quad-1.41559 \quad 2.09839 \quad-0.91276$

S9 $\quad-0.89077 \quad-1.37046 \quad 0.12451$

$\begin{array}{llll}\mathrm{O} 10 & -1.76089 & -1.88578 & 1.15481\end{array}$

$\begin{array}{llll}011 & -1.33562 & -0.98872 & -1.19712\end{array}$

$\begin{array}{llll}\mathrm{C} 12 & 0.50816 & -2.59559 & -0.08694\end{array}$

$\begin{array}{llll}\text { F13 } & 0.00000 & -3.71132 & -0.58361\end{array}$

$\begin{array}{llll}\text { F14 } & 1.06594 & -2.83570 & 1.09348\end{array}$ 
$\begin{array}{llll}\text { F15 } & 1.41559 & -2.09839 & -0.91276\end{array}$

2) Table $\mathrm{S} 1$

\begin{tabular}{lccc} 
1c-cis- $a$ & \multicolumn{4}{c}{} \\
MP2 & -4611.1037707 & au & \\
C1 & -0.28648 & 0.00000 & 0.16229 \\
S2 & -0.68082 & -1.55438 & 0.80492 \\
O3 & 0.39534 & -2.46757 & 0.44033 \\
O4 & -1.20792 & -1.44351 & 2.14549 \\
C5 & -2.10797 & -2.13680 & -0.24298 \\
F6 & -2.38773 & -3.39974 & 0.05900 \\
F7 & -1.77137 & -2.06576 & -1.53848 \\
F8 & -3.18255 & -1.38680 & -0.03874 \\
S9 & -0.68082 & 1.55440 & 0.80489 \\
O10 & -1.20793 & 1.44354 & 2.14546 \\
O11 & 0.39535 & 2.46757 & 0.44030 \\
C12 & -2.10795 & 2.13680 & -0.24302 \\
F13 & -2.38772 & 3.39975 & 0.05893 \\
F14 & -3.18254 & 1.38682 & -0.03879 \\
F15 & -1.77134 & 2.06574 & -1.53852 \\
Br16 & 0.92036 & -0.00001 & -1.25860 \\
C17 & 2.66374 & -0.00001 & -0.44443 \\
C18 & 3.24998 & -1.23535 & -0.17249 \\
C19 & 3.24998 & 1.23534 & -0.17252 \\
C20 & 4.52015 & -1.21539 & 0.41558 \\
C21 & 4.52015 & 1.21539 & 0.41555 \\
C22 & 5.15090 & 0.00000 & 0.70620 \\
H23 & 2.72873 & -2.16311 & -0.38160 \\
H24 & 2.72873 & 2.16310 & -0.38165 \\
H25 & 5.00895 & -2.15598 & 0.65095 \\
H26 & 5.00896 & 2.15599 & 0.65091 \\
H27 & 6.13392 & 0.00001 & 1.16718
\end{tabular}

1c- $c i s-b$

MP2 $=-4611.100755 \mathrm{au}$

$\begin{array}{llrl}\text { C1 } & -0.71940 & -0.02362 & -0.81357 \\ \text { S2 } & -1.24268 & 1.62753 & -0.69223 \\ \text { O3 } & -0.20031 & 2.43261 & -1.32180 \\ \text { O4 } & -2.64546 & 1.79444 & -0.99440 \\ \text { C5 } & -1.09075 & 2.03463 & 1.12215 \\ \text { F6 } & -1.26515 & 3.34470 & 1.27660 \\ \text { F7 } & 0.12865 & 1.70953 & 1.56337 \\ \text { F8 } & -1.99933 & 1.38278 & 1.83118 \\ \text { S9 } & -1.65410 & -1.41982 & -0.43163 \\ \text { O10 } & -2.99641 & -1.00345 & -0.08645 \\ \text { O11 } & -1.34952 & -2.48268 & -1.37569\end{array}$




$\begin{array}{lrrc}\text { C12 } & -0.91498 & -2.05697 & 1.15908 \\ \text { F13 } & -1.56319 & -3.15265 & 1.53589 \\ \text { F14 } & -0.98651 & -1.15036 & 2.12646 \\ \text { F15 } & 0.37658 & -2.36556 & 0.96361 \\ \text { Br16 } & 0.86064 & -0.31197 & -1.75450 \\ \text { C17 } & 2.29478 & -0.08277 & -0.49402 \\ \text { C18 } & 2.93521 & -1.22160 & -0.00714 \\ \text { C19 } & 2.64517 & 1.22399 & -0.15446 \\ \text { C20 } & 3.98669 & -1.02562 & 0.89589 \\ \text { C21 } & 3.69284 & 1.38343 & 0.75953 \\ \text { C22 } & 4.36102 & 0.26772 & 1.27809 \\ \text { H23 } & 2.62096 & -2.21654 & -0.30391 \\ \text { H24 } & 2.10786 & 2.07084 & -0.56920 \\ \text { H25 } & 4.50663 & -1.88876 & 1.30051 \\ \text { H26 } & 3.98801 & 2.38535 & 1.05644 \\ \text { H27 } & 5.17336 & 0.40626 & 1.98501\end{array}$

3) Table $\mathrm{S} 2$

$9 c$ (TS)-trans

$\mathrm{MP} 2=-4611.048117 \mathrm{au}$

$\begin{array}{llll}\mathrm{C} 1 & -1.01322 & 0.55100 & 0.34714\end{array}$

$\begin{array}{llll}\text { S2 } & -1.77342 & -0.97880 & -0.33988\end{array}$

$\begin{array}{llll}\text { O3 } & -1.68253 & -1.12683 & -1.78085\end{array}$

$\begin{array}{llll}\text { O4 } & -1.45686 & -2.06615 & 0.56857\end{array}$

$\begin{array}{llll}\text { C5 } & -3.54816 & -0.51434 & 0.02247\end{array}$

$\begin{array}{llll}\text { F6 } & -4.29706 & -1.54894 & -0.34940\end{array}$

$\begin{array}{llll}\text { F7 } & -3.90839 & 0.55877 & -0.66436\end{array}$

$\begin{array}{llll}\text { F8 } & -3.71678 & -0.29421 & 1.31874\end{array}$

$\begin{array}{llll}\text { S9 } & 0.02861 & 1.25763 & -0.80377\end{array}$

$\begin{array}{llll}\mathrm{O} 10 & 1.03099 & 0.72067 & -1.69097\end{array}$

$\begin{array}{llll}\text { O11 } & -1.33778 & 1.71528 & -1.24954\end{array}$

$\begin{array}{llll}\mathrm{C} 12 & 0.81623 & 2.74630 & 0.02109\end{array}$

$\begin{array}{llll}\text { F13 } & 0.94187 & 3.66596 & -0.92932\end{array}$

$\begin{array}{llll}\text { F14 } & 2.01506 & 2.41929 & 0.47995\end{array}$

$\begin{array}{llll}\text { F15 } & 0.06372 & 3.20788 & 0.99765\end{array}$

$\begin{array}{llll}\text { Br16 } & 1.04654 & -0.55418 & 1.89865\end{array}$

$\begin{array}{llll}\mathrm{C} 17 & 2.15667 & -1.20669 & 0.49486\end{array}$

$\begin{array}{llll}\mathrm{C} 18 & 1.72617 & -2.30747 & -0.25057\end{array}$

$\begin{array}{llll}\text { C19 } & 3.34940 & -0.53566 & 0.21473\end{array}$

$\begin{array}{llll}\mathrm{C} 20 & 2.51342 & -2.72459 & -1.32961\end{array}$

$\begin{array}{llll}\mathrm{C} 21 & 4.12325 & -0.97555 & -0.86463\end{array}$

$\begin{array}{llll}\mathrm{C} 22 & 3.70387 & -2.05969 & -1.64263\end{array}$

$\begin{array}{llll}\mathrm{H} 23 & 0.80191 & -2.81628 & 0.00104\end{array}$

$\begin{array}{llrr}\mathrm{H} 24 & 3.66212 & 0.30952 & 0.81885\end{array}$

$\begin{array}{llll}\mathrm{H} 25 & 2.18602 & -3.57163 & -1.92555\end{array}$

$\mathrm{H} 26 \quad 5.05142 \quad-0.46120 \quad-1.09706$ 
4) Table $S 3$

\begin{tabular}{|c|c|c|c|}
\hline \multicolumn{4}{|c|}{ trans ${ }^{-1} \mathbf{1} \mathbf{c}-a$} \\
\hline $\mathrm{C} 1$ & 0.00000 & 0.00000 & 0.83135 \\
\hline $\mathrm{S} 2$ & 0.89077 & 1.37046 & 0.12451 \\
\hline $\mathrm{O} 3$ & 1.76089 & 1.88578 & 1.15481 \\
\hline $\mathrm{O} 4$ & 1.33562 & 0.98872 & -1.19712 \\
\hline $\mathrm{C} 5$ & -0.50816 & 2.59559 & -0.08694 \\
\hline F6 & 0.00000 & 3.71132 & -0.58361 \\
\hline F7 & -1.06594 & 2.83570 & 1.09348 \\
\hline F8 & -1.41559 & 2.09839 & -0.91276 \\
\hline S9 & -0.89077 & -1.37046 & 0.12451 \\
\hline $\mathrm{O} 10$ & -1.76089 & -1.88578 & 1.15481 \\
\hline O11 & -1.33562 & -0.98872 & -1.19712 \\
\hline $\mathrm{C} 12$ & 0.50816 & -2.59559 & -0.08694 \\
\hline $\mathrm{F} 13$ & 0.00000 & -3.71132 & -0.58361 \\
\hline F14 & 1.06594 & -2.83570 & 1.09348 \\
\hline F15 & 1.41559 & -2.09839 & -0.91276 \\
\hline
\end{tabular}

trans $-{ }^{1} \mathbf{1} \mathbf{c}-b$

MP2 $=-1807.395828$ au

$\begin{array}{lccc}\text { C1 } & 0.16175 & 1.04435 & -0.09497 \\ \text { S2 } & 1.08126 & -0.36402 & -0.12435 \\ \text { O3 } & 0.95503 & -1.76370 & 0.20198 \\ \text { O4 } & 0.66670 & 0.23012 & -1.51163 \\ \text { C5 } & 2.87871 & 0.11146 & 0.13798 \\ \text { F6 } & 3.61782 & -0.81267 & -0.45151 \\ \text { F7 } & 3.10057 & 0.11309 & 1.44182 \\ \text { F8 } & 3.11914 & 1.29169 & -0.37813 \\ \text { S9 } & -1.60102 & 1.01069 & 0.14668 \\ \text { O10 } & -1.76066 & 1.37543 & 1.53973 \\ \text { O11 } & -2.17835 & 1.73023 & -0.96546 \\ \text { C12 } & -2.28619 & -0.73026 & 0.01623 \\ \text { F13 } & -3.60699 & -0.66567 & 0.08183 \\ \text { F14 } & -1.82640 & -1.47255 & 1.01923 \\ \text { F15 } & -1.92317 & -1.28462 & -1.13876\end{array}$

\begin{tabular}{|c|c|c|c|}
\hline \multicolumn{4}{|c|}{${ }^{1} \mathbf{1 c}(\mathrm{TS}-t t)$} \\
\hline \multicolumn{4}{|c|}{$\mathrm{MP} 2=-1807.3912026 \mathrm{au}$} \\
\hline $\mathrm{C} 1$ & -0.10332 & 1.02688 & -0.45984 \\
\hline $\mathrm{S} 2$ & -1.06981 & -0.03323 & 0.45528 \\
\hline $\mathrm{O} 3$ & -0.92546 & -1.22554 & 1.25728 \\
\hline $\mathrm{O} 4$ & -0.88155 & 1.39823 & 1.03252 \\
\hline $\mathrm{C} 5$ & -2.76897 & -0.09470 & -0.34378 \\
\hline
\end{tabular}




\begin{tabular}{|c|c|c|c|}
\hline F6 & -3.63729 & -0.42641 & 0.59608 \\
\hline F7 & -2.72891 & -1.02801 & -1.28106 \\
\hline F8 & -3.07213 & 1.07139 & -0.86037 \\
\hline S9 & 1.70116 & 0.94245 & -0.10523 \\
\hline $\mathrm{O} 10$ & 2.31592 & 1.46454 & -1.30552 \\
\hline $\mathrm{O} 11$ & 1.98232 & 1.43396 & 1.22810 \\
\hline $\mathrm{C} 12$ & 2.11384 & -0.89442 & -0.12339 \\
\hline F13 & 3.36477 & -1.05466 & -0.51608 \\
\hline F14 & 1.29337 & -1.51086 & -0.98781 \\
\hline F15 & 1.94898 & -1.42294 & 1.07839 \\
\hline \multicolumn{4}{|c|}{${ }^{1} \mathbf{1 c}(\mathrm{TS}-c t)$} \\
\hline \multicolumn{4}{|c|}{$\mathrm{MP} 2=-1807.384615 \mathrm{au}$} \\
\hline $\mathrm{C} 1$ & -0.10332 & 1.02688 & -0.45984 \\
\hline S2 & -1.06981 & -0.03323 & 0.45528 \\
\hline $\mathrm{O} 3$ & -0.92546 & -1.22554 & 1.25728 \\
\hline $\mathrm{O} 4$ & -0.88155 & 1.39823 & 1.03252 \\
\hline $\mathrm{C} 5$ & -2.76897 & -0.09470 & -0.34378 \\
\hline F6 & -3.63729 & -0.42641 & 0.59608 \\
\hline F7 & -2.72891 & -1.02801 & -1.28106 \\
\hline F8 & -3.07213 & 1.07139 & -0.86037 \\
\hline S9 & 1.70116 & 0.94245 & -0.10523 \\
\hline $\mathrm{O} 10$ & 2.31592 & 1.46454 & -1.30552 \\
\hline $\mathrm{O} 11$ & 1.98232 & 1.43396 & 1.22810 \\
\hline $\mathrm{C} 12$ & 2.11384 & -0.89442 & -0.12339 \\
\hline F13 & 3.36477 & -1.05466 & -0.51608 \\
\hline F14 & 1.29337 & -1.51086 & -0.98781 \\
\hline F15 & 1.94898 & -1.42294 & 1.07839 \\
\hline
\end{tabular}

\section{5) Table $\mathrm{S} 4$}

\begin{tabular}{lccc} 
cis- ${ }^{3}$ 1c & \multicolumn{3}{l}{} \\
MP2 $=-1807.3920086$ & au & \\
C1 & -0.00899 & -0.79493 & 0.70262 \\
S2 & -1.47636 & -0.87012 & -0.30309 \\
O3 & -1.09085 & -0.69898 & -1.68609 \\
O4 & -2.29971 & -1.95102 & 0.18358 \\
C5 & -2.27427 & 0.72921 & 0.24625 \\
F6 & -3.41702 & 0.86297 & -0.40518 \\
F7 & -1.46819 & 1.74610 & -0.03813 \\
F8 & -2.49560 & 0.69489 & 1.55358 \\
S9 & 1.73084 & -0.84913 & 0.34596 \\
O10 & 2.41032 & -0.99734 & 1.61073 \\
O11 & 1.93258 & -1.71320 & -0.79459 \\
C12 & 2.01568 & 0.91096 & -0.22565 \\
F13 & 3.31048 & 1.05980 & -0.45176 \\
F14 & 1.62169 & 1.75087 & 0.72560
\end{tabular}




\begin{tabular}{|c|c|c|c|}
\hline F15 & 1.32808 & 1.14326 & -1.33236 \\
\hline \multicolumn{4}{|c|}{${ }^{3} 1 \mathrm{c}(\mathrm{TS}-c c)$} \\
\hline \multicolumn{4}{|c|}{$\mathrm{MP} 2=-1807.390632 \mathrm{au}$} \\
\hline $\mathrm{C} 1$ & 0.00000 & -0.79233 & -0.66676 \\
\hline $\mathrm{S} 2$ & 1.65549 & -0.92209 & -0.02426 \\
\hline $\mathrm{O} 3$ & 1.59677 & -1.36927 & 1.34774 \\
\hline $\mathrm{O} 4$ & 2.44857 & -1.55234 & -1.05278 \\
\hline $\mathrm{C} 5$ & 2.13663 & 0.88527 & -0.00218 \\
\hline F6 & 3.42173 & 0.96052 & 0.29953 \\
\hline F7 & 1.41798 & 1.53202 & 0.90472 \\
\hline F8 & 1.91784 & 1.41765 & -1.19961 \\
\hline S9 & -1.65549 & -0.92209 & -0.02426 \\
\hline $\mathrm{O} 10$ & -2.44856 & -1.55234 & -1.05277 \\
\hline O11 & -1.59676 & -1.36927 & 1.34775 \\
\hline $\mathrm{C} 12$ & -2.13663 & 0.88527 & -0.00219 \\
\hline F13 & -3.42173 & 0.96051 & 0.29952 \\
\hline F14 & -1.91783 & 1.41764 & -1.19961 \\
\hline F15 & -1.41799 & 1.53202 & 0.90472 \\
\hline \multicolumn{4}{|c|}{${ }^{3} \mathbf{1 c}(\mathrm{TS}-c t)$} \\
\hline \multicolumn{4}{|c|}{$\mathrm{MP} 2=-1807.390143 \mathrm{au}$} \\
\hline $\mathrm{C} 1$ & -0.04151 & 0.36656 & -0.30629 \\
\hline $\mathrm{S} 2$ & -1.35009 & -0.49322 & 0.54136 \\
\hline $\mathrm{O} 3$ & -1.24618 & -1.89909 & 0.22177 \\
\hline $\mathrm{O} 4$ & -1.45645 & 0.01858 & 1.88815 \\
\hline $\mathrm{C} 5$ & -2.78803 & 0.19064 & -0.43720 \\
\hline F6 & -3.89439 & -0.36712 & 0.02549 \\
\hline F7 & -2.63313 & -0.10365 & -1.72195 \\
\hline F8 & -2.84616 & 1.50718 & -0.28792 \\
\hline S9 & 1.57360 & 0.93050 & 0.15965 \\
\hline $\mathrm{O} 10$ & 1.93675 & 1.98300 & -0.75865 \\
\hline O11 & 1.62717 & 1.04835 & 1.59871 \\
\hline $\mathrm{C} 12$ & 2.56503 & -0.58809 & -0.29569 \\
\hline F13 & 3.83283 & -0.35634 & 0.00134 \\
\hline F14 & 2.43907 & -0.83548 & -1.59240 \\
\hline F15 & 2.11518 & -1.62437 & 0.39979 \\
\hline
\end{tabular}


7. Spectra

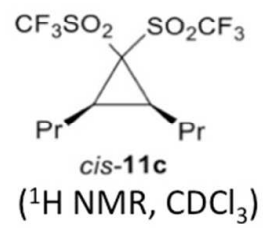

$\left({ }^{1} \mathrm{H} \mathrm{NMR}, \mathrm{CDCl}_{3}\right)$
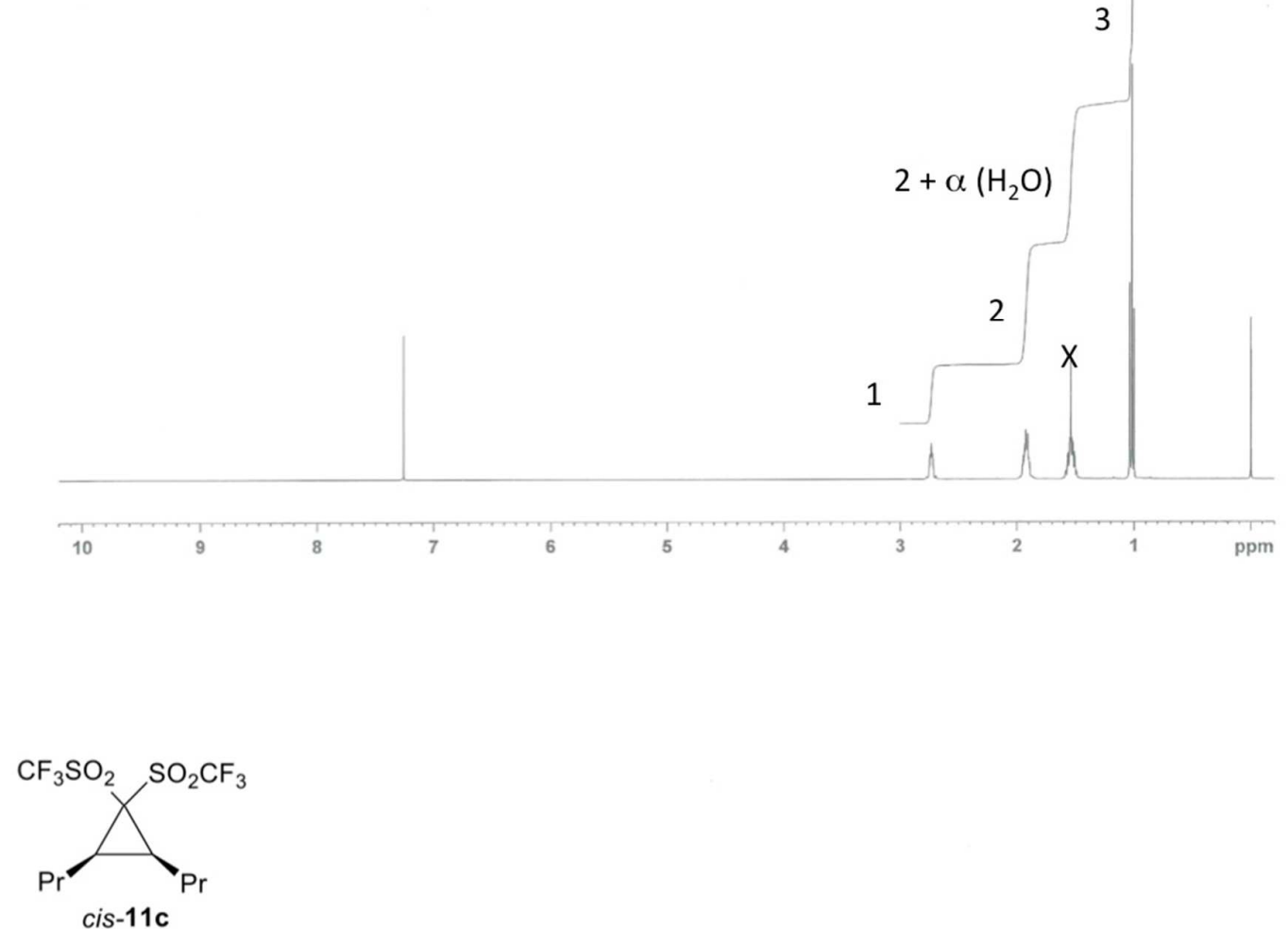

$\left({ }^{13} \mathrm{C} \mathrm{NMR}, \mathrm{CDCl}_{3}\right)$

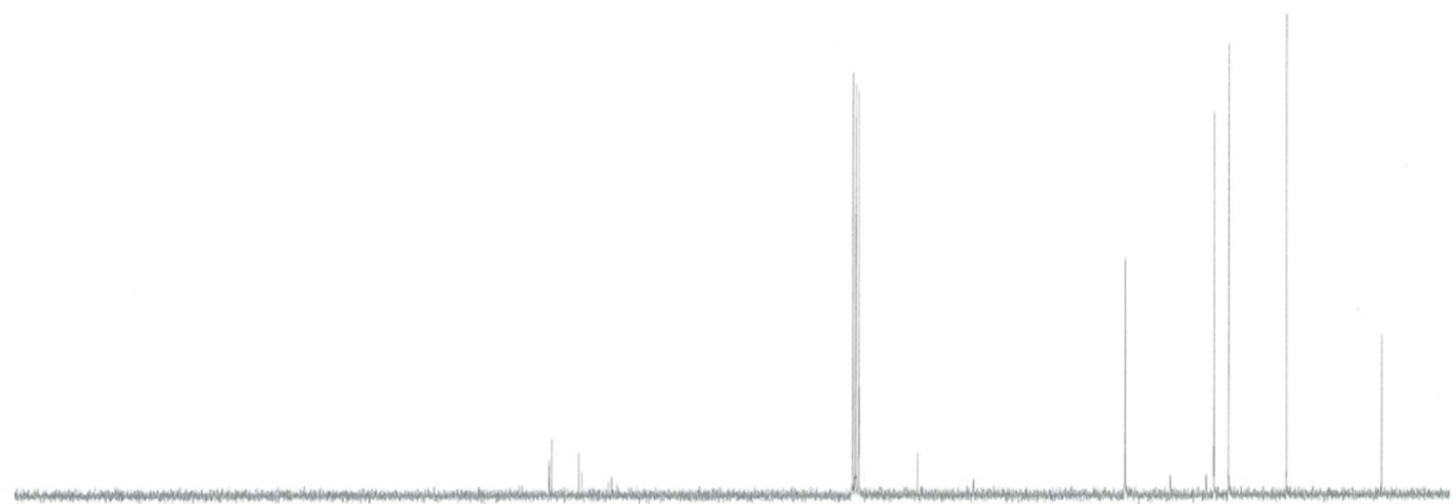

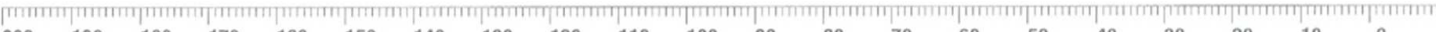

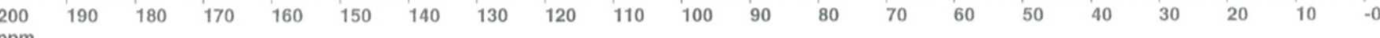




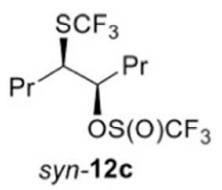

$\left({ }^{1} \mathrm{H} \mathrm{NMR,} \mathrm{CDCl}_{3}\right)$

1:1 mixture of diastereomers
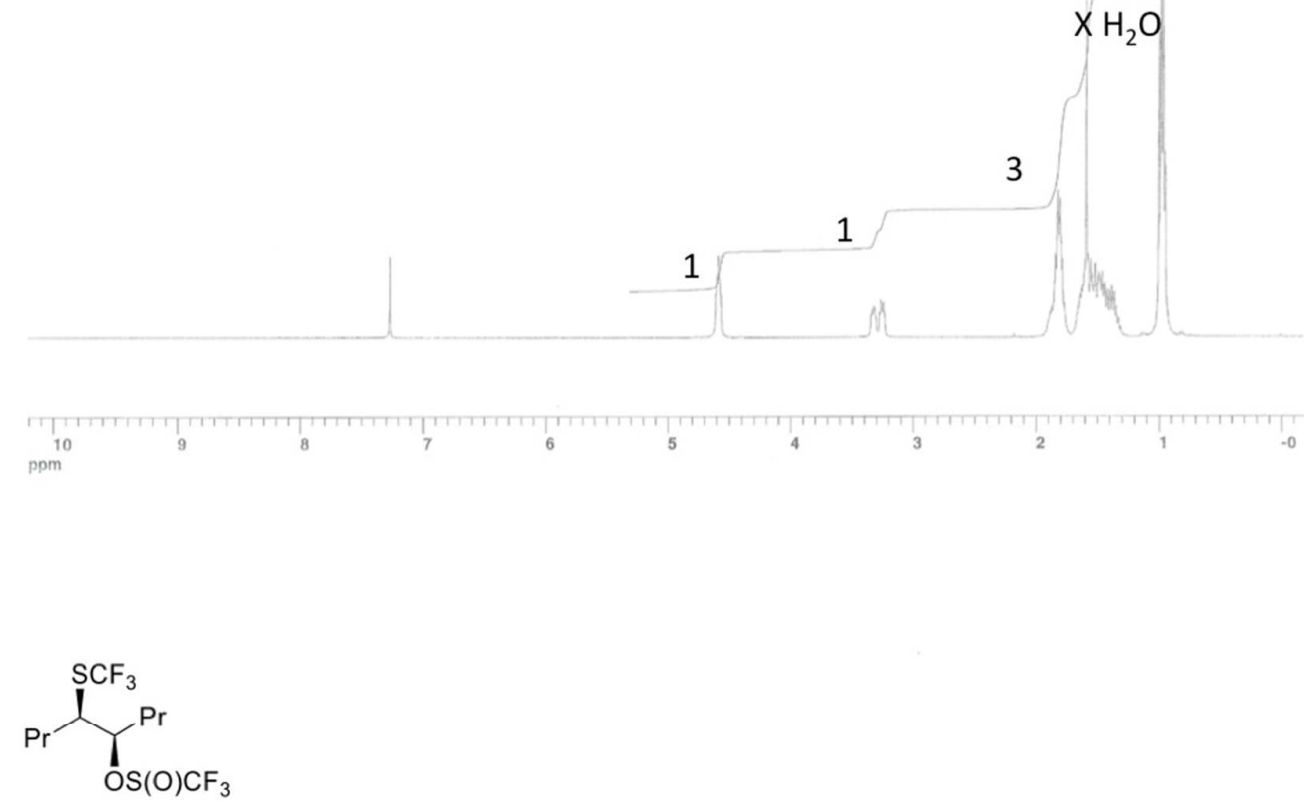

syn-12c

$\left({ }^{13} \mathrm{C} \mathrm{NMR,} \mathrm{CDCl}_{3}\right)$

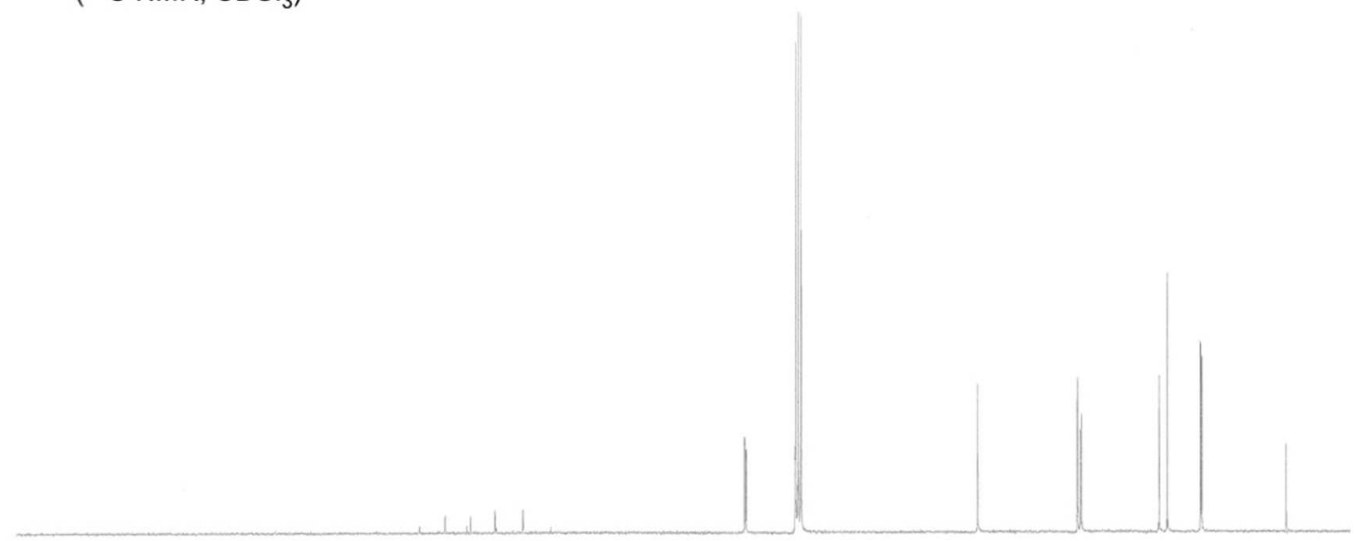

[ 200

$\begin{array}{llllllllllllllllllll}90 & 180 & 170 & 160 & 150 & 140 & 130 & 120 & 110 & 100 & 90 & 80 & 70 & 60 & 50 & 40 & 30 & 20 & 10 & -0\end{array}$ 


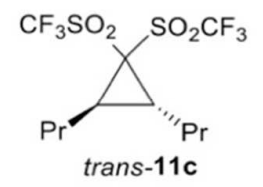

$\left({ }^{1} \mathrm{H} \mathrm{NMR}, \mathrm{CDCl}_{3}\right)$
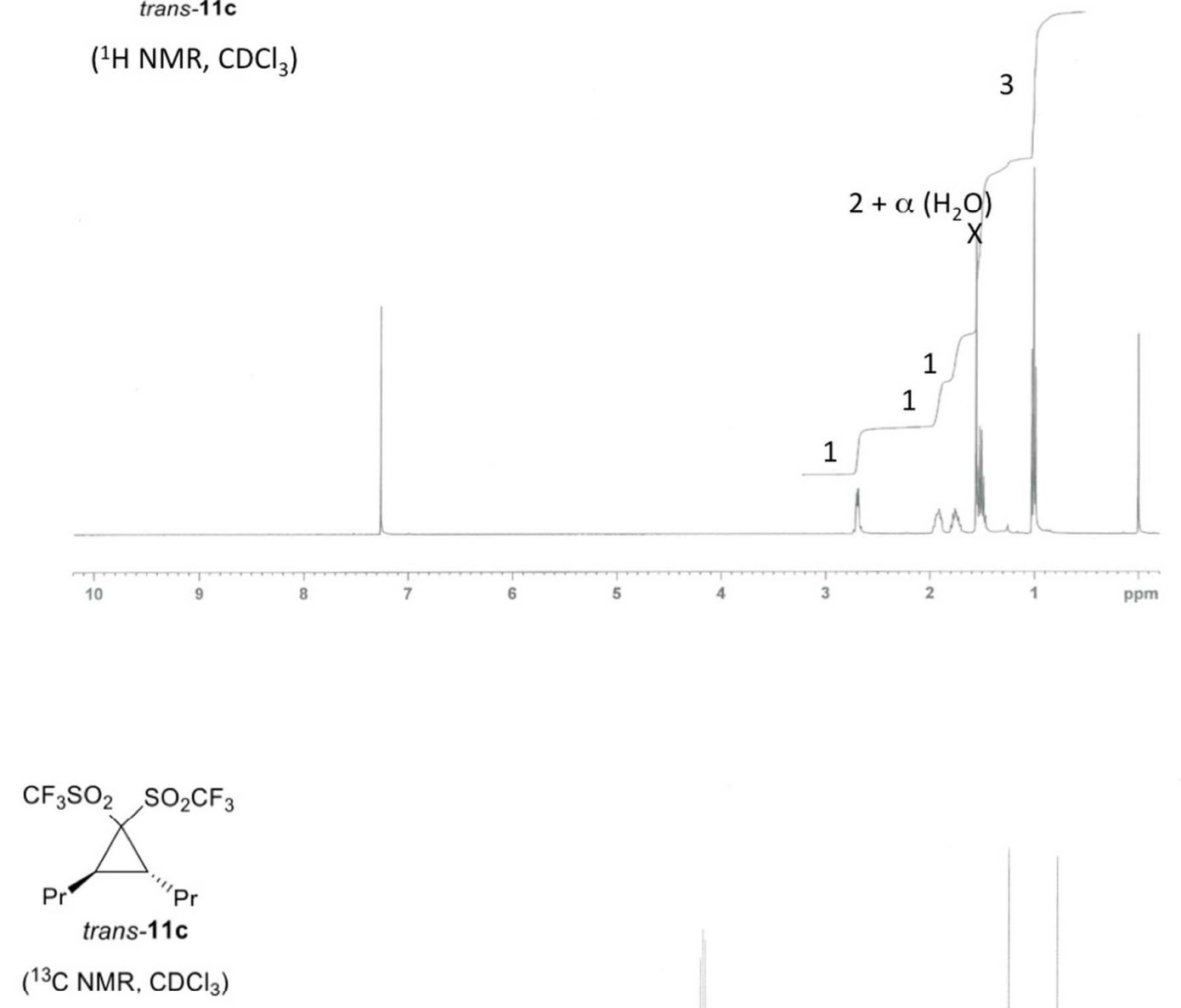

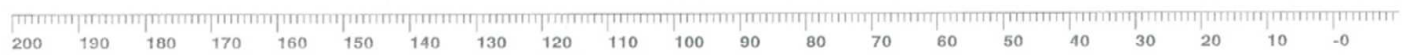
$\mathrm{ppm}$ 


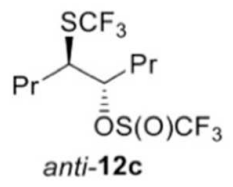

$\left({ }^{1} \mathrm{H} N M R, \mathrm{CDCl}_{3}\right)$

1:1 mixture of diastereomers

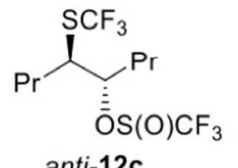

$\left({ }^{13} \mathrm{C}\right.$ NMR, $\left.\mathrm{CDCl}_{3}\right)$

\section{$X \mathrm{H}_{2} \mathrm{O}$}

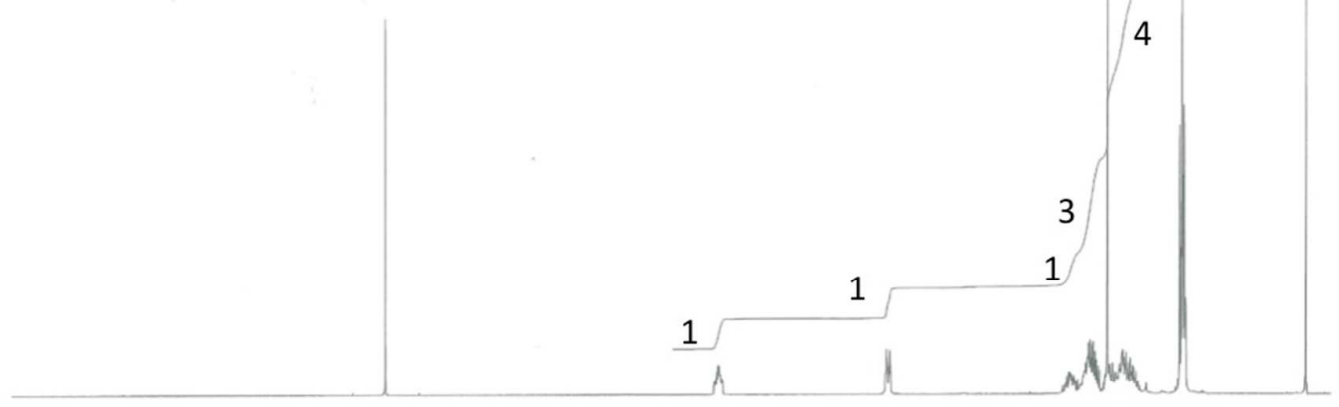

$\begin{array}{lllllllllll}10 & 9 & 8 & 7 & 6 & 5 & 4 & 3 & 2 & 1 & \mathrm{ppm}\end{array}$

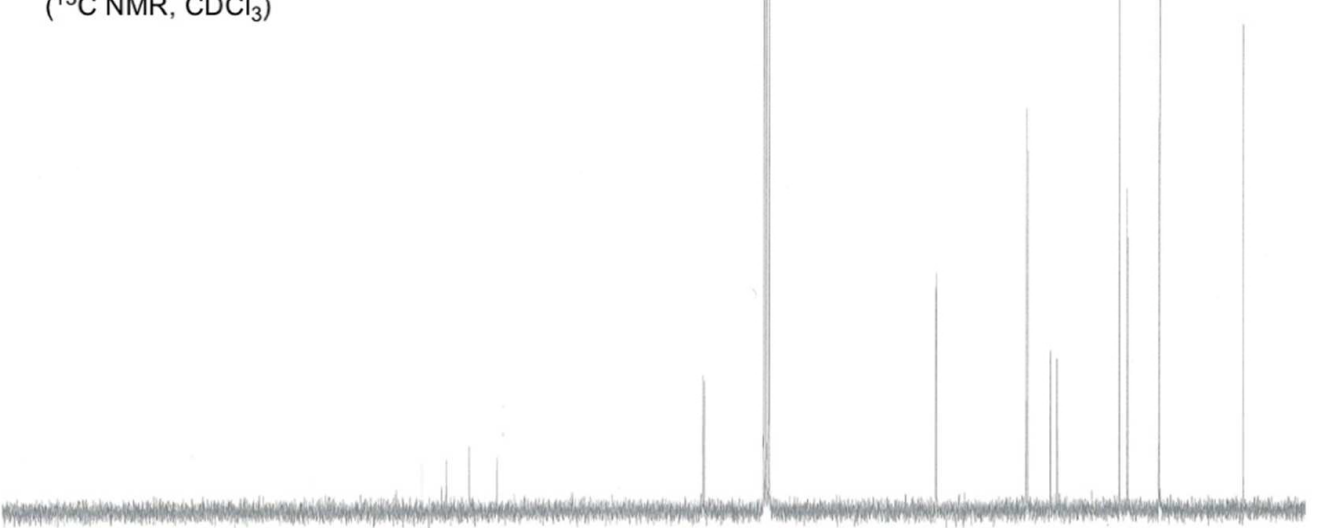

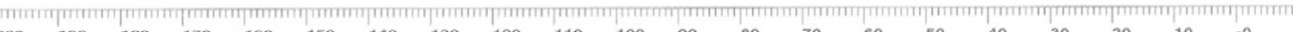
$\begin{array}{llllllllllllllllllllll}200 & 190 & 180 & 170 & 160 & 150 & 140 & 130 & 120 & 110 & 100 & 90 & 80 & 70 & 60 & 50 & 40 & 30 & 20 & 10 & -0\end{array}$ $\mathrm{ppm}$ 


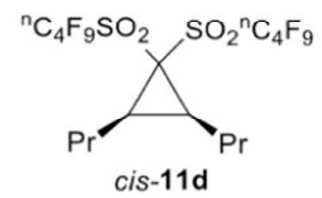

( ${ }^{1} \mathrm{H}$ NMR, $\mathrm{CDCl}_{3}$ )
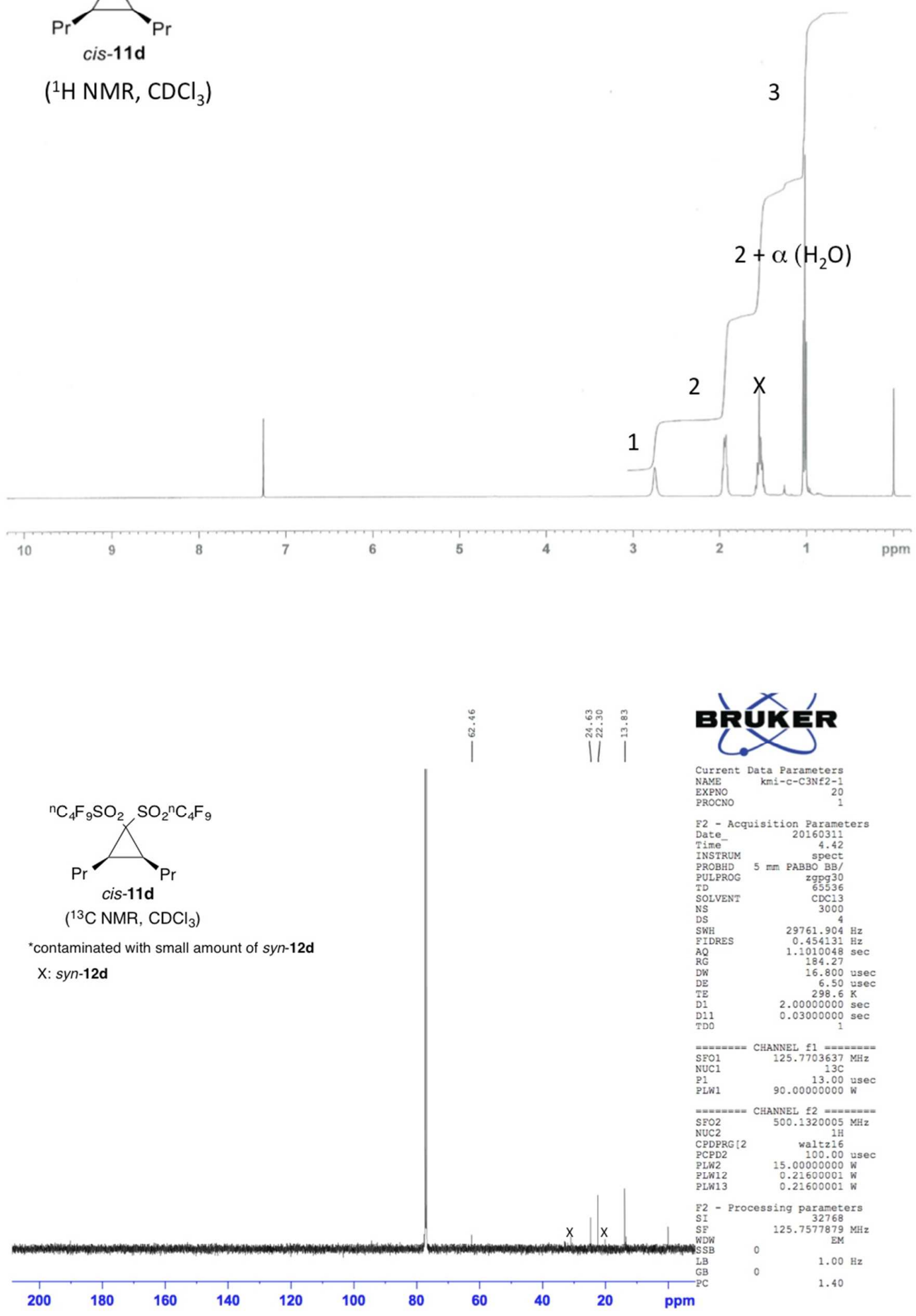
$\overbrace{\text { syn-12d }}^{\left.{ }^{1} \mathrm{H} \mathrm{NMR}, \mathrm{CDCl}_{3}\right)} \mathrm{C}_{4}^{\mathrm{n}} \mathrm{C}_{4} \mathrm{~F}_{9}$

2:1 mixture of diastereomers
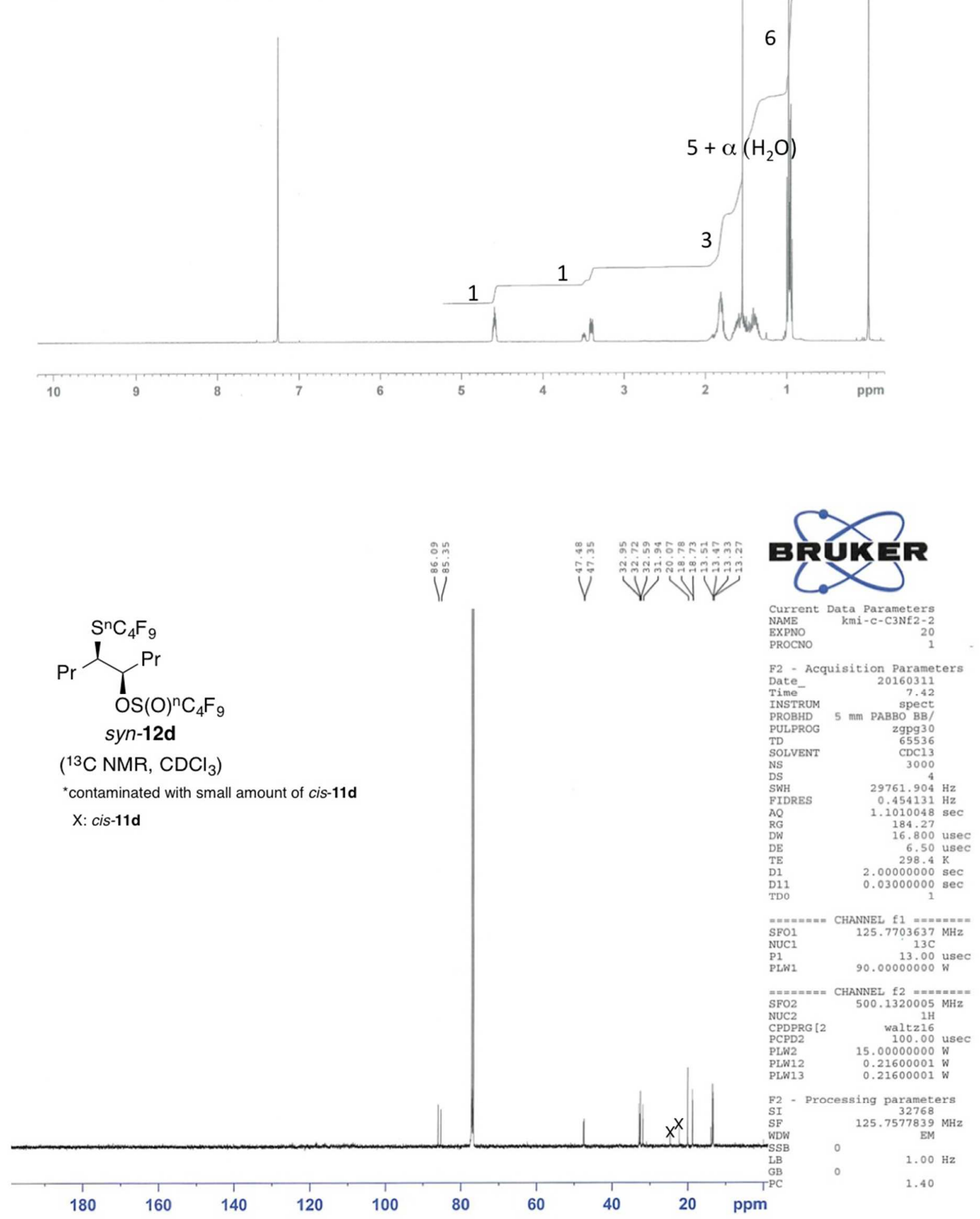

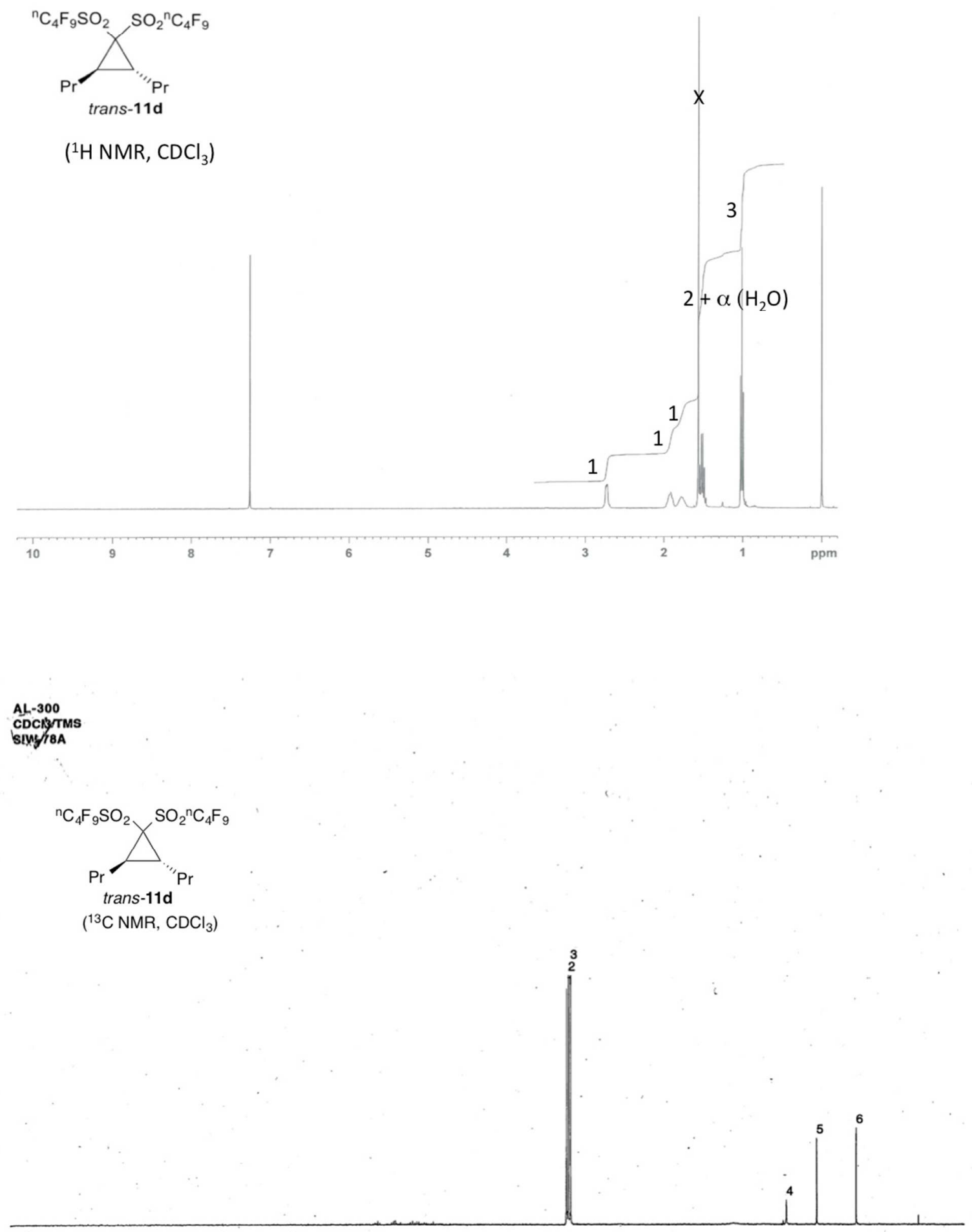

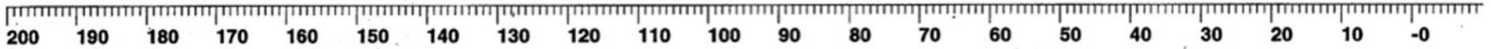

200
ppm 


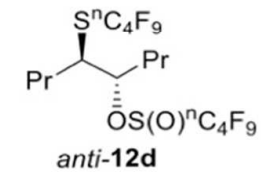

$\left({ }^{1} \mathrm{H} \mathrm{NMR}, \mathrm{CDCl}_{3}\right.$ )

1:1 mixture of diastereomers
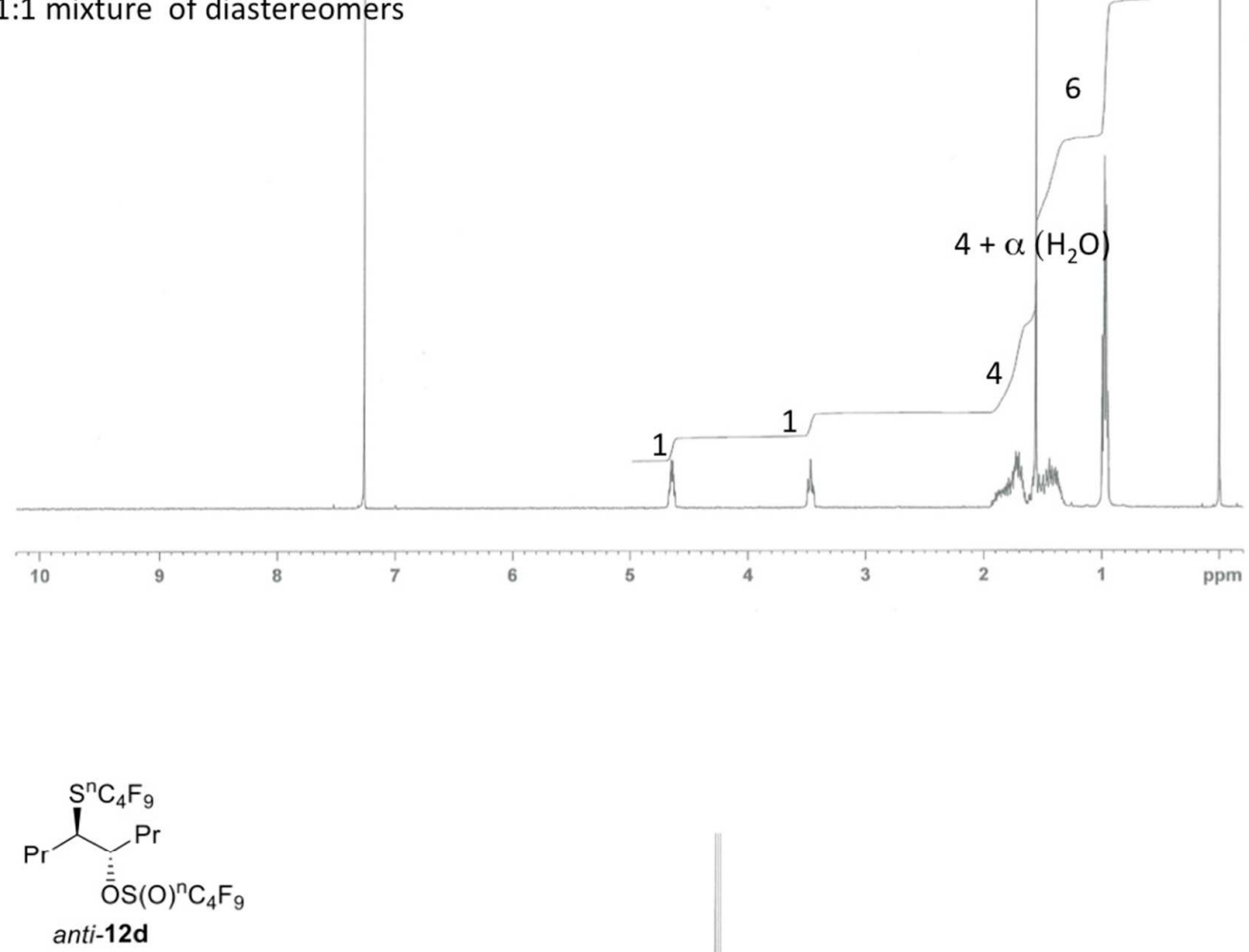

$\left({ }^{13} \mathrm{C} \mathrm{NMR,}, \mathrm{CDCl}_{3}\right)$
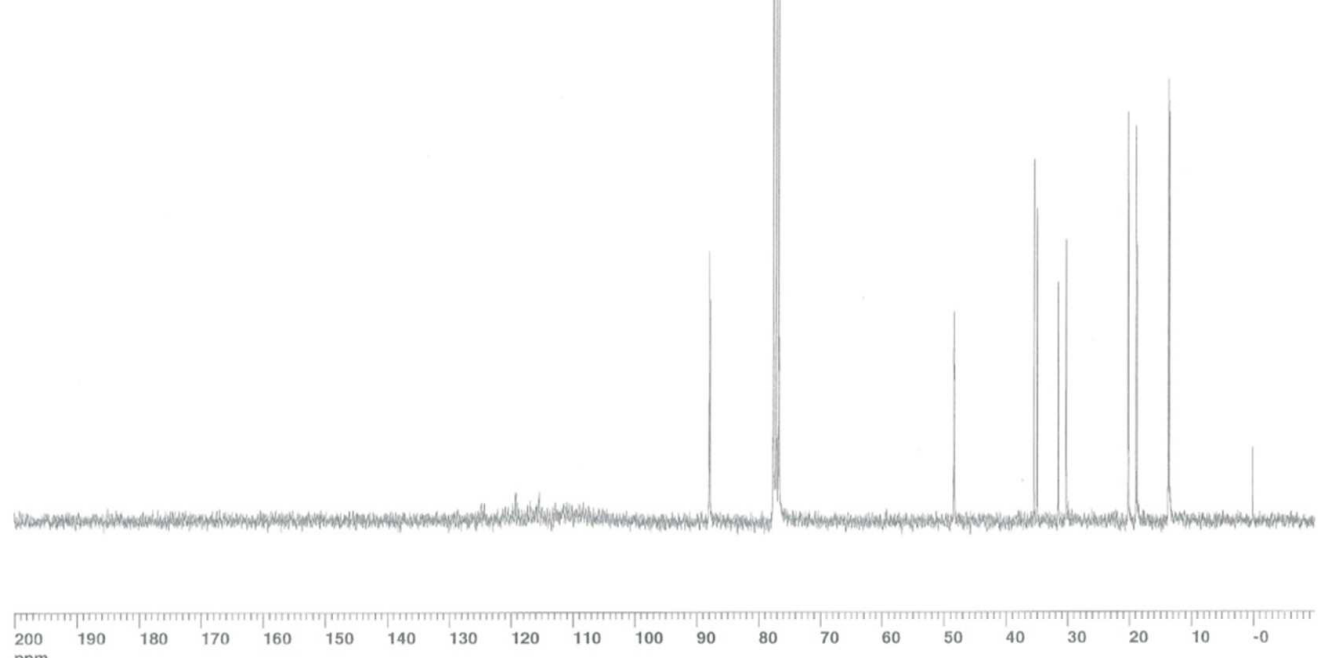

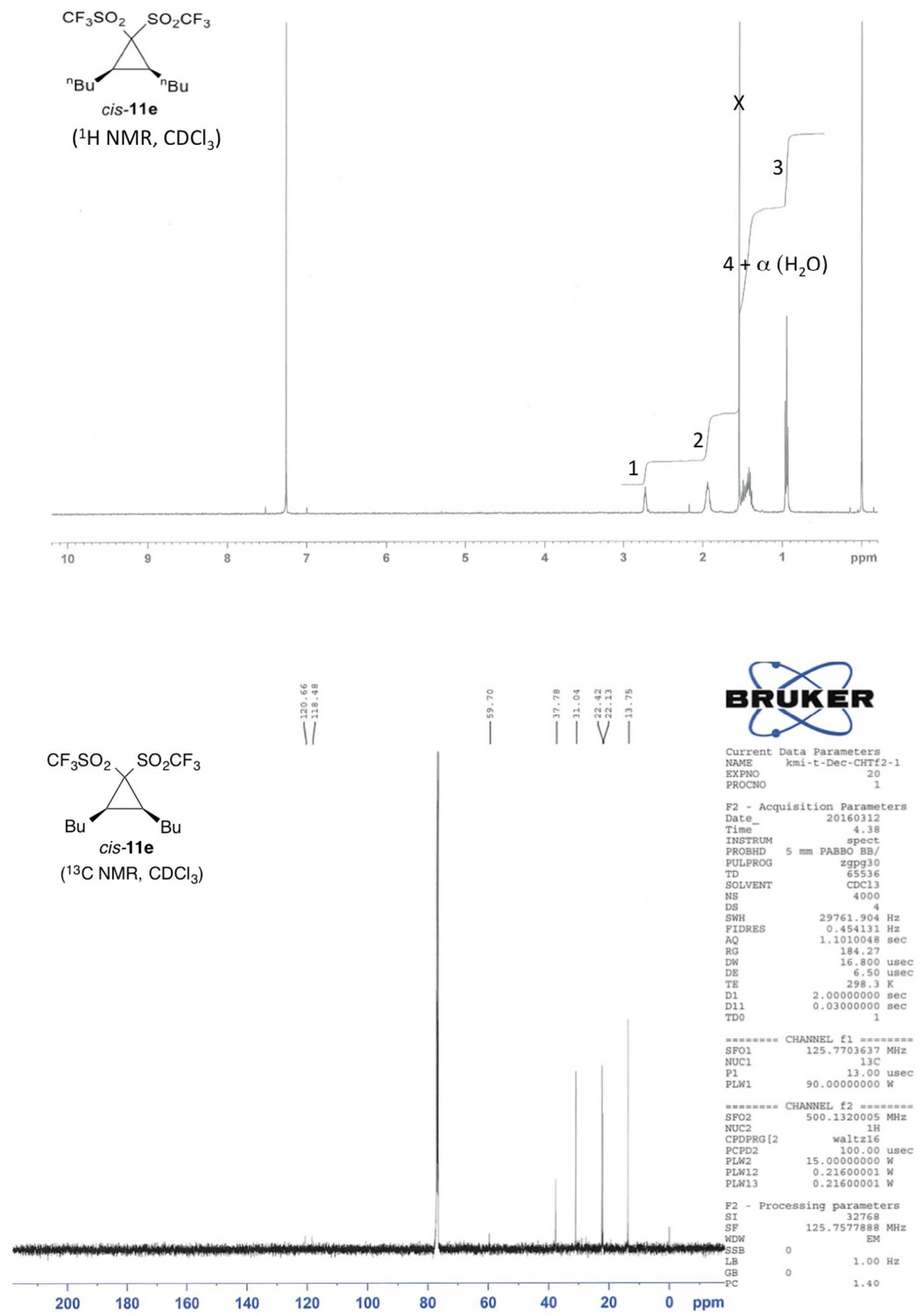


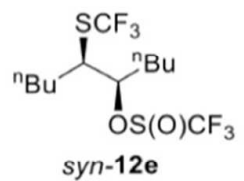

( ${ }^{1} \mathrm{H} \mathrm{NMR}, \mathrm{CDCl}_{3}$ )

2:1 mixture of diastereomers

6

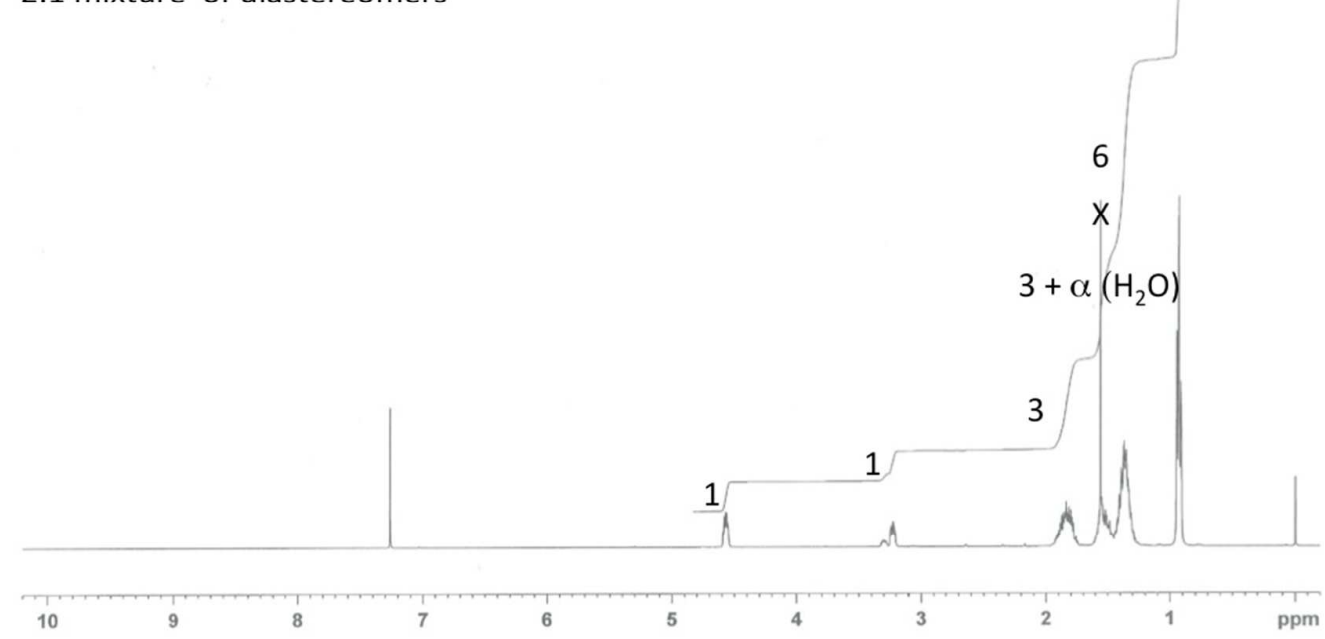

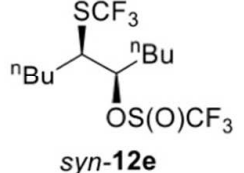

syn-12e

$\left({ }^{13} \mathrm{C} \mathrm{NMR}, \mathrm{CDCl}_{3}\right)$

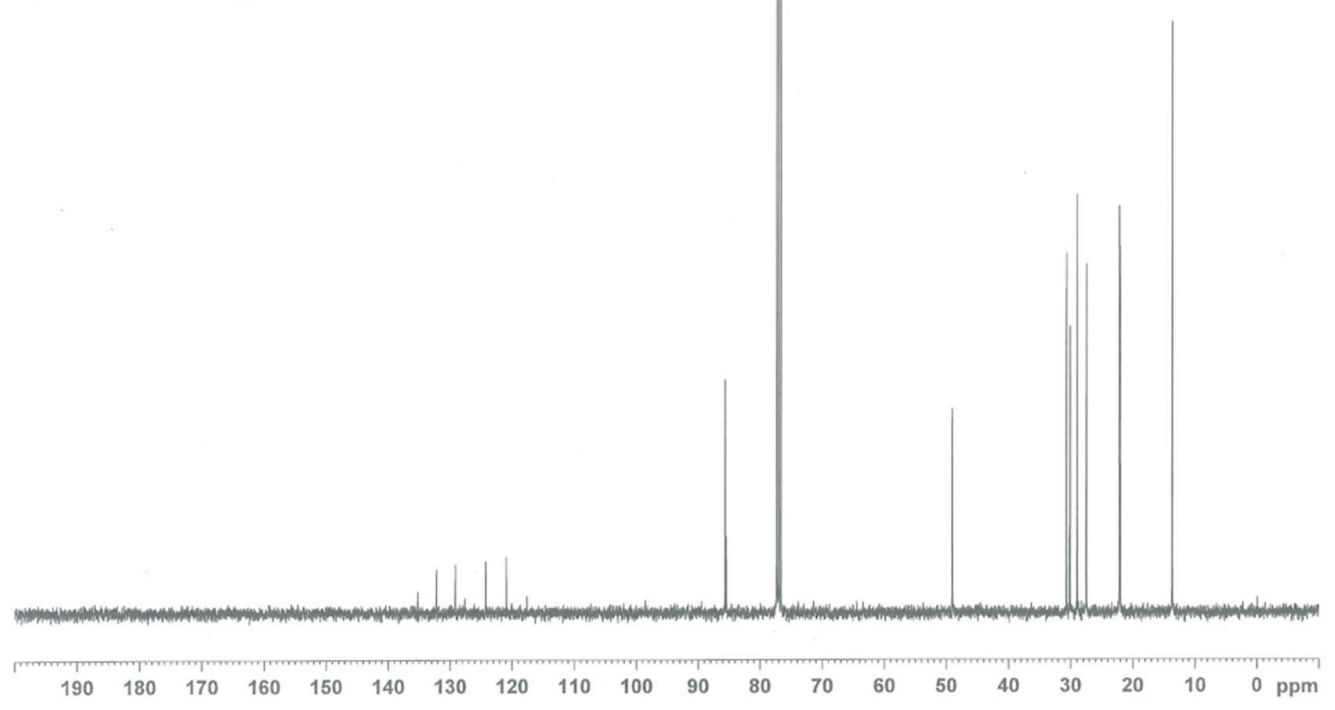




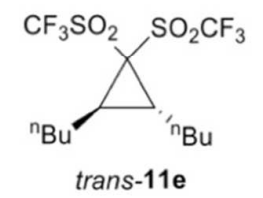

$\left({ }^{1} \mathrm{H} \mathrm{NMR}, \mathrm{CDCl}_{3}\right)$
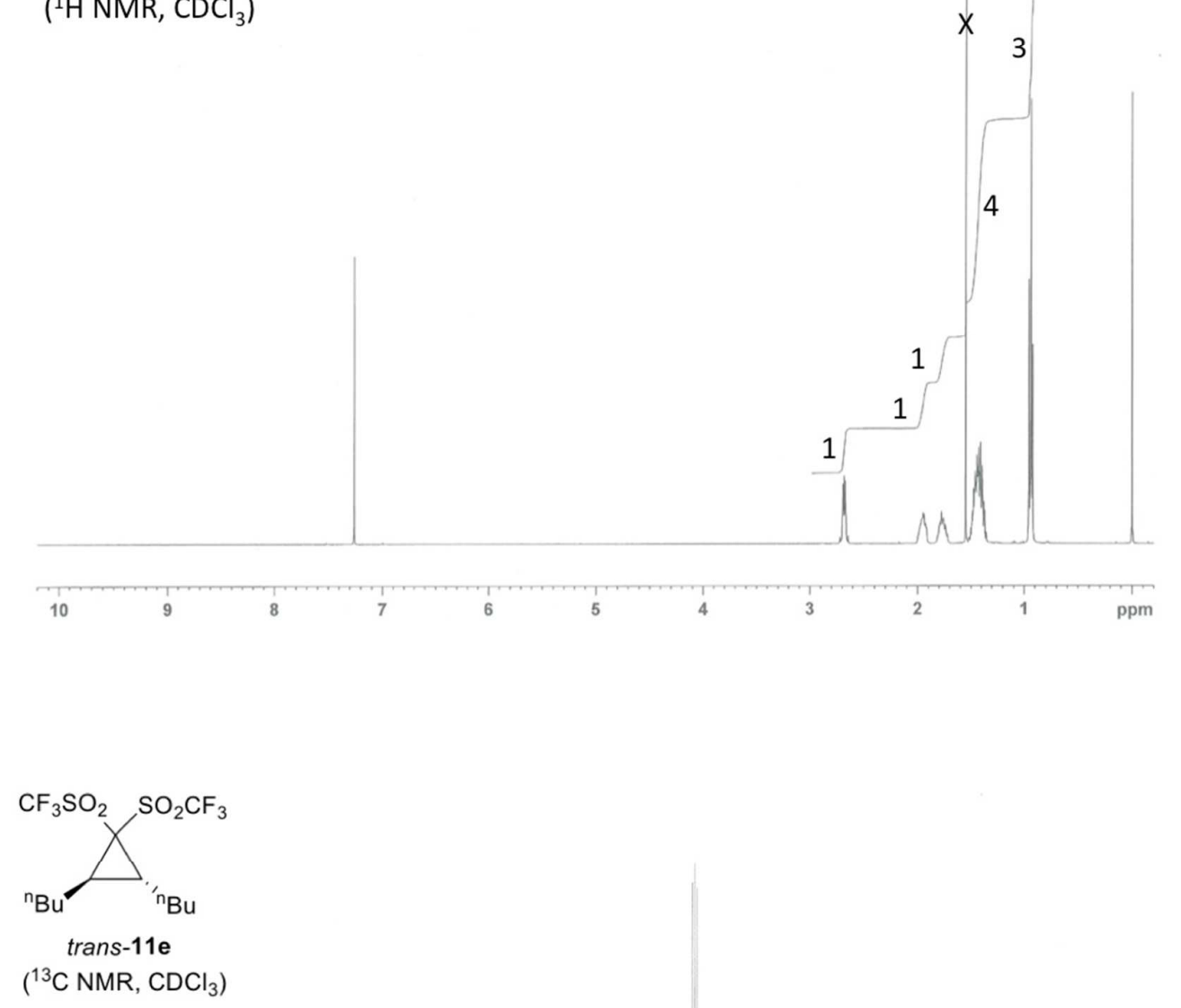


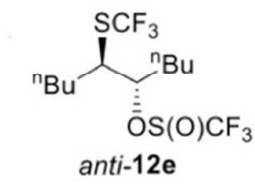

$\left({ }^{1} \mathrm{H} \mathrm{NMR}, \mathrm{CDCl}_{3}\right)$

1:1 mixture of diastereomers
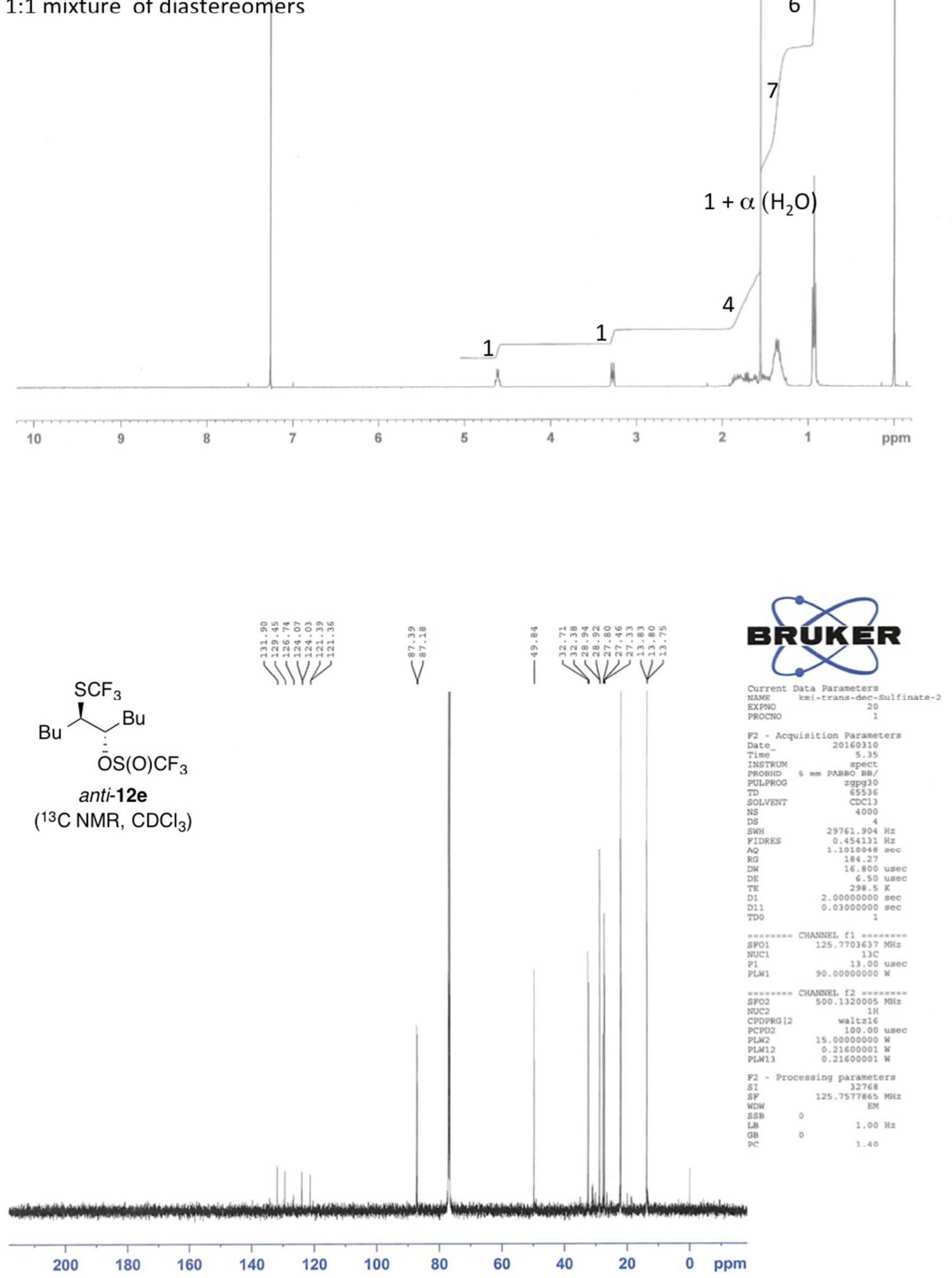

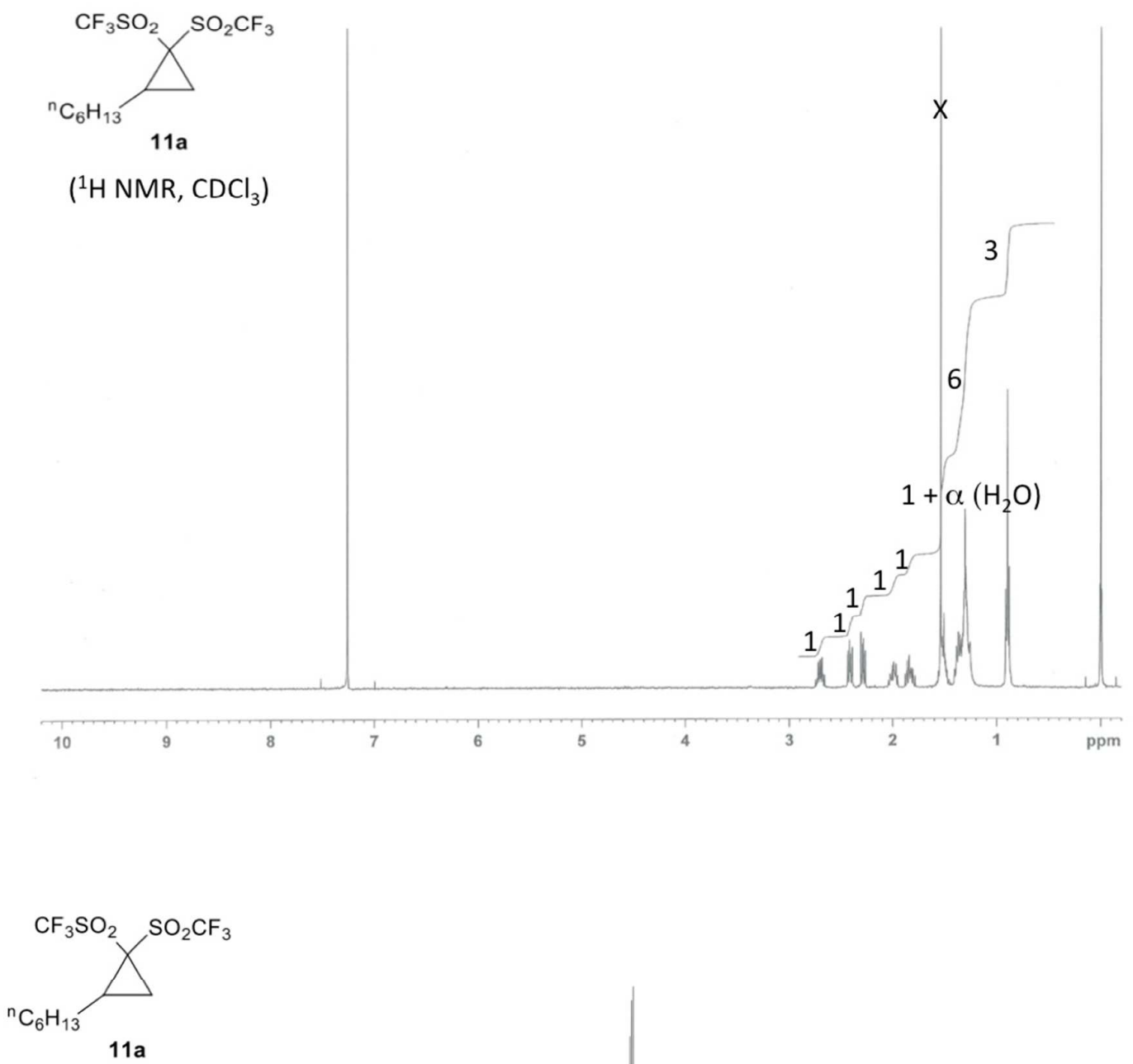

$\left({ }^{13} \mathrm{CNMR} \mathrm{CDCl}_{3}\right)$ 

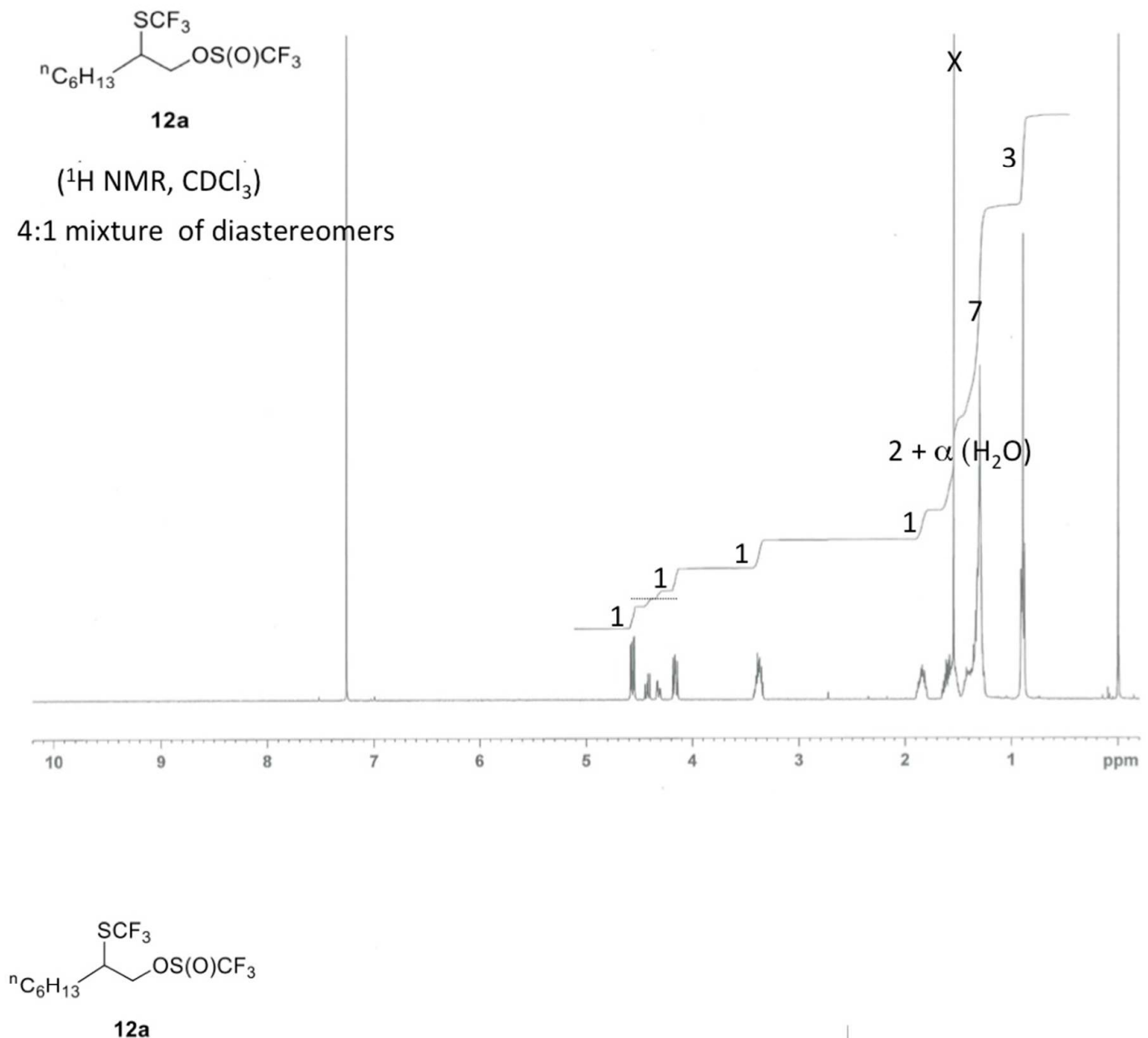

$\left({ }^{13} \mathrm{C} \mathrm{NMR,} \mathrm{CDCl}_{3}\right)$

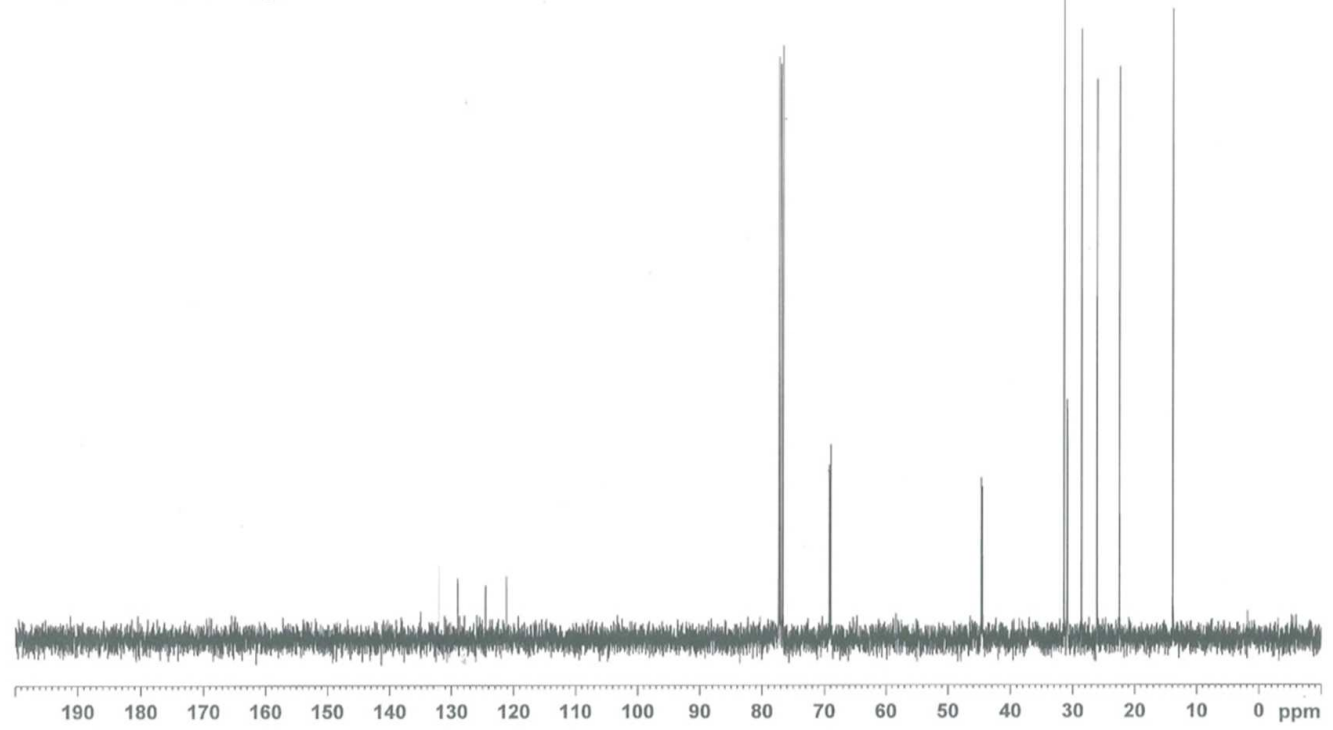



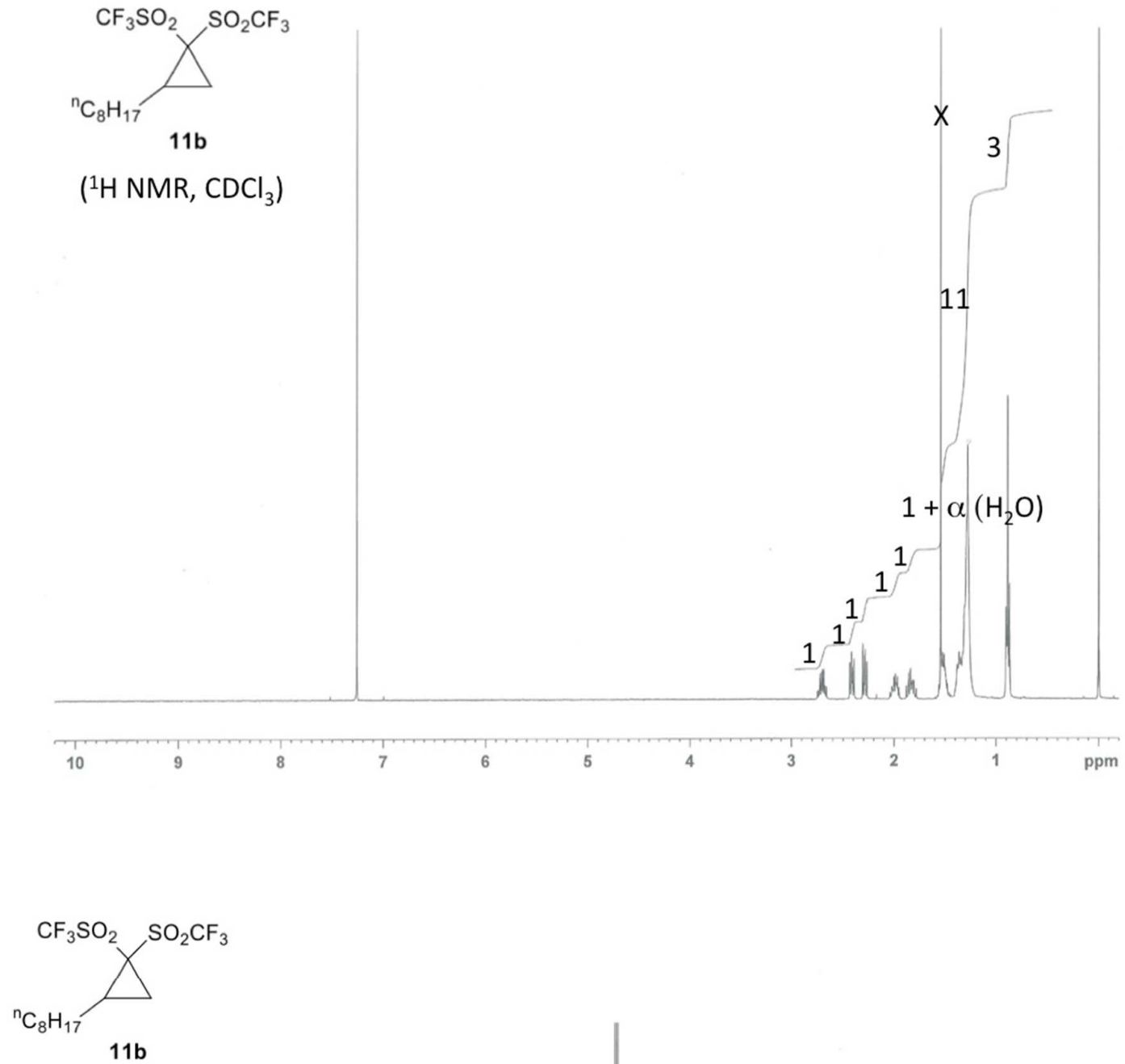

$\left({ }^{13} \mathrm{C} \mathrm{NMR}, \mathrm{CDCl}_{3}\right)$ 
${ }^{n} \mathrm{C}_{8} \mathrm{H}_{17} \overbrace{12 \mathrm{~b}}^{\mathrm{SCF}_{3}} \mathrm{OS}(\mathrm{O}) \mathrm{CF}_{3}$

( ${ }^{1} \mathrm{H} N M R, \mathrm{CDCl}_{3}$ )

1:1 mixture of diastereomers

$1: 1$ mixture of diastereomers

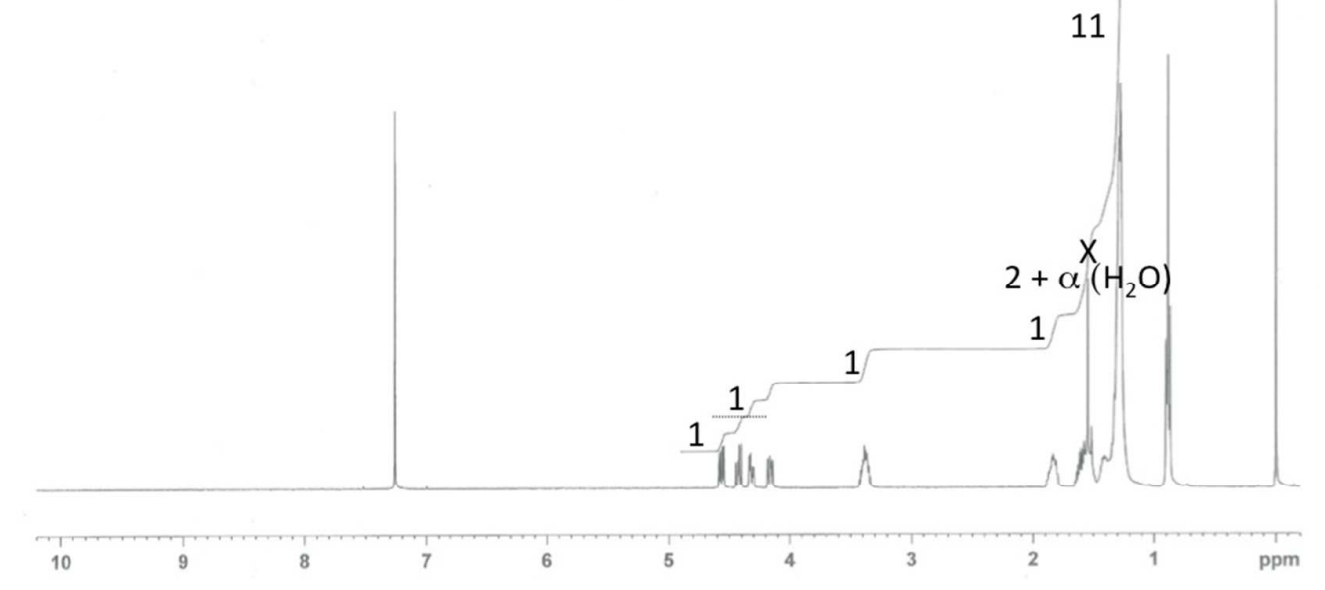

$\left({ }^{13} \mathrm{CNMR}, \mathrm{CDCl}_{3}\right)$

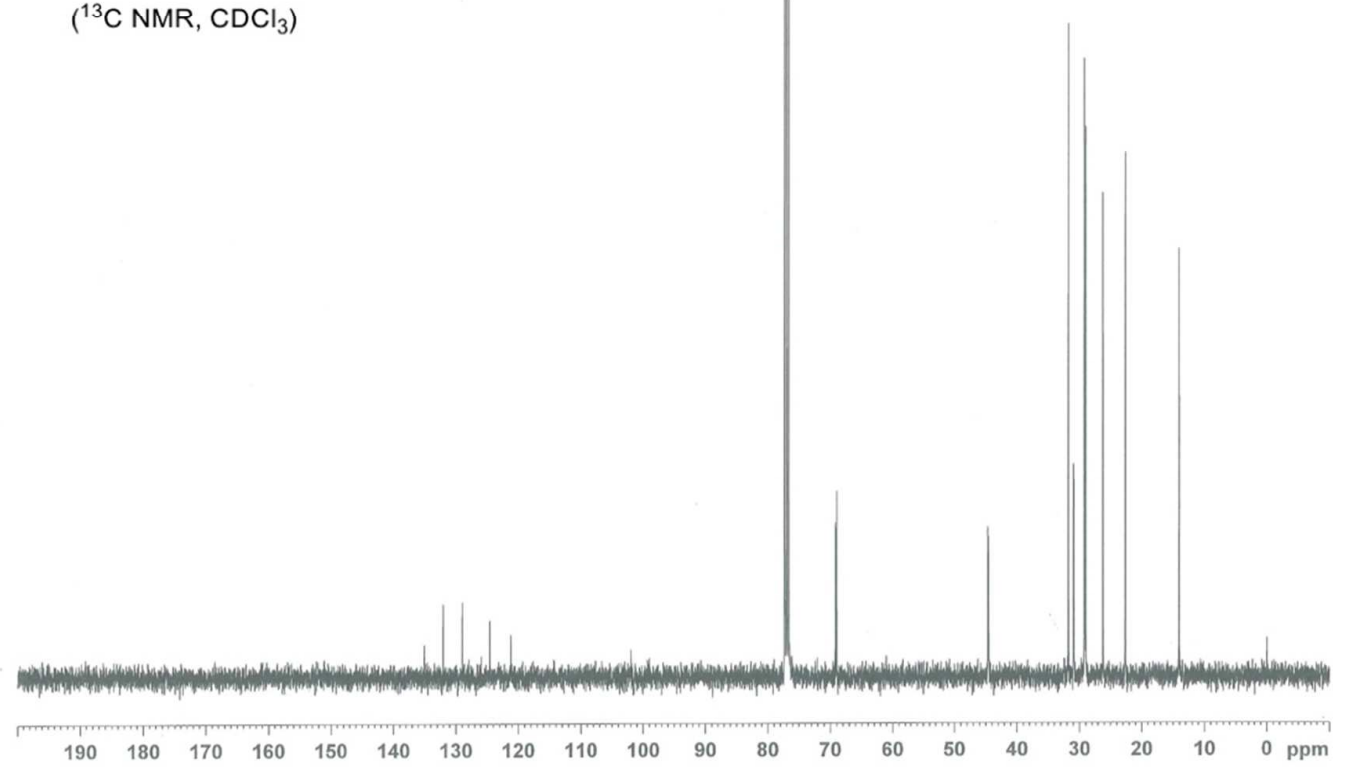




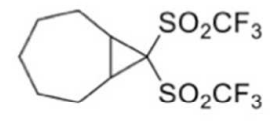

$11 f$

$\left({ }^{1} \mathrm{H} N M R, \mathrm{CDCl}_{3}\right)$

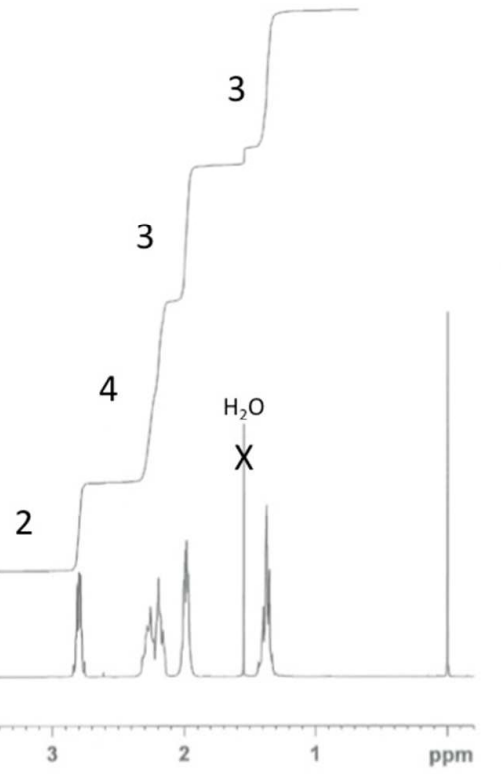

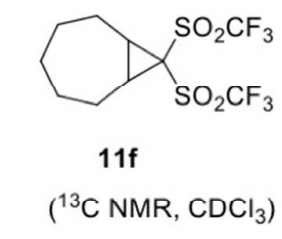

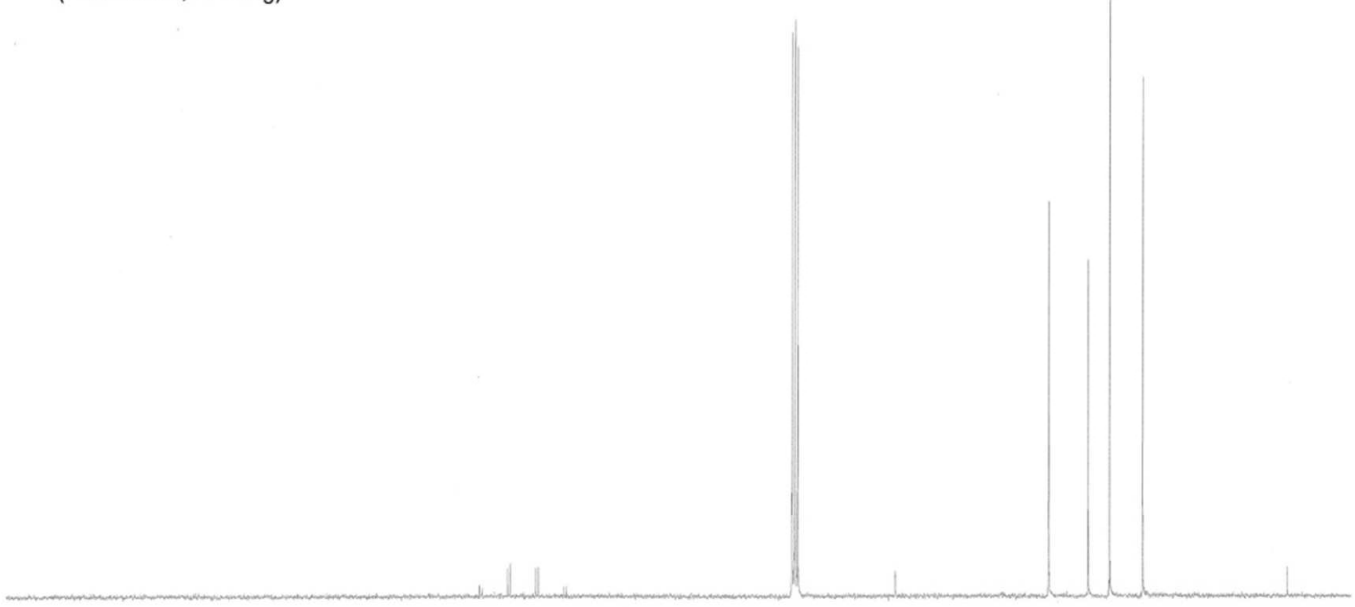

|

\section{4}

$\mathrm{ppm}$

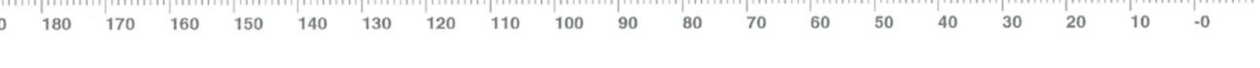




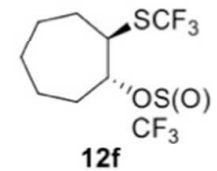

$\left({ }^{1} \mathrm{H} N M R, \mathrm{CDCl}_{3}\right)$

$6: 4$ mixture of diastereomers
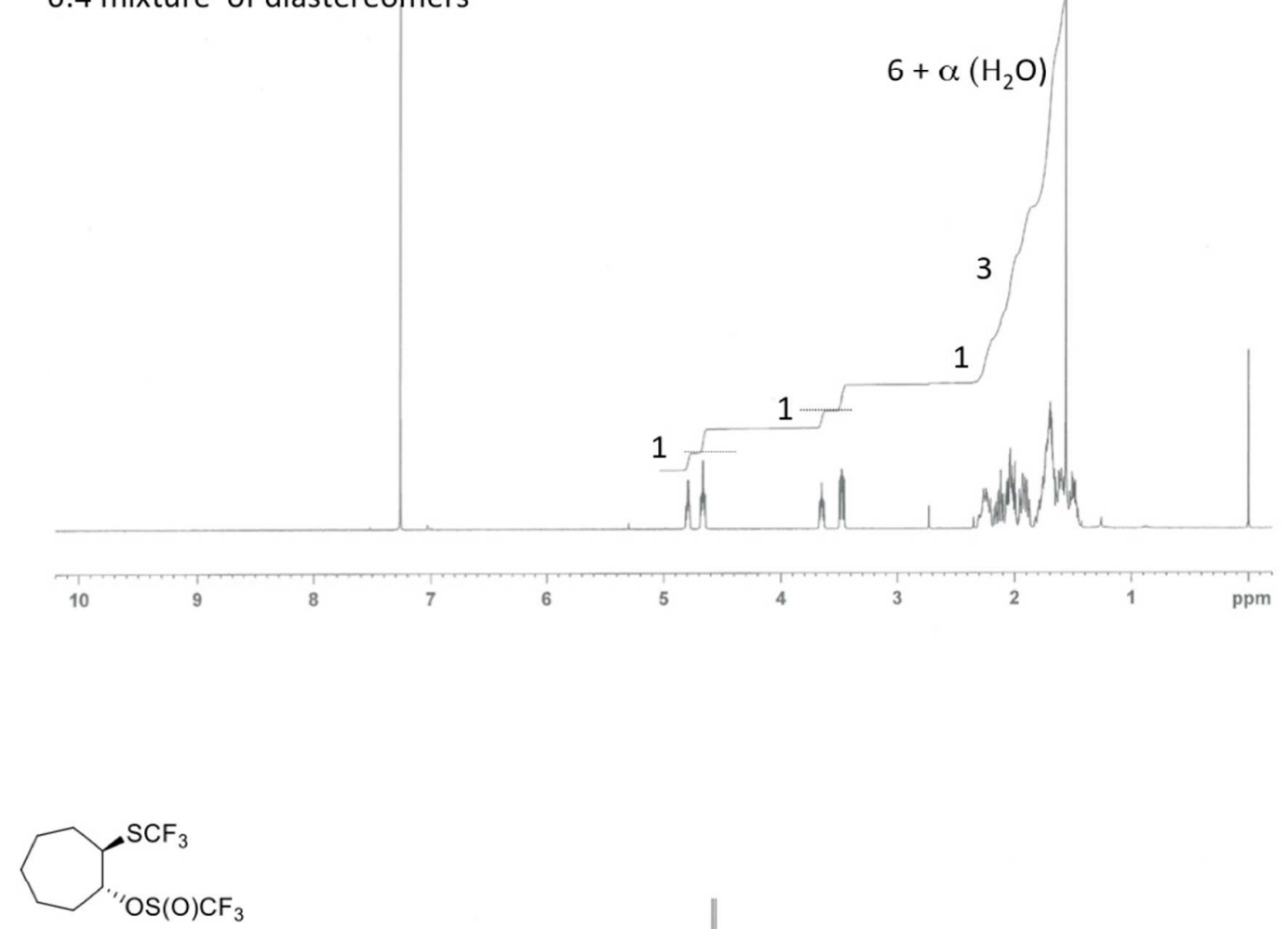

$12 f$

$\left({ }^{13} \mathrm{C} \mathrm{NMR,} \mathrm{CDCl}_{3}\right)$

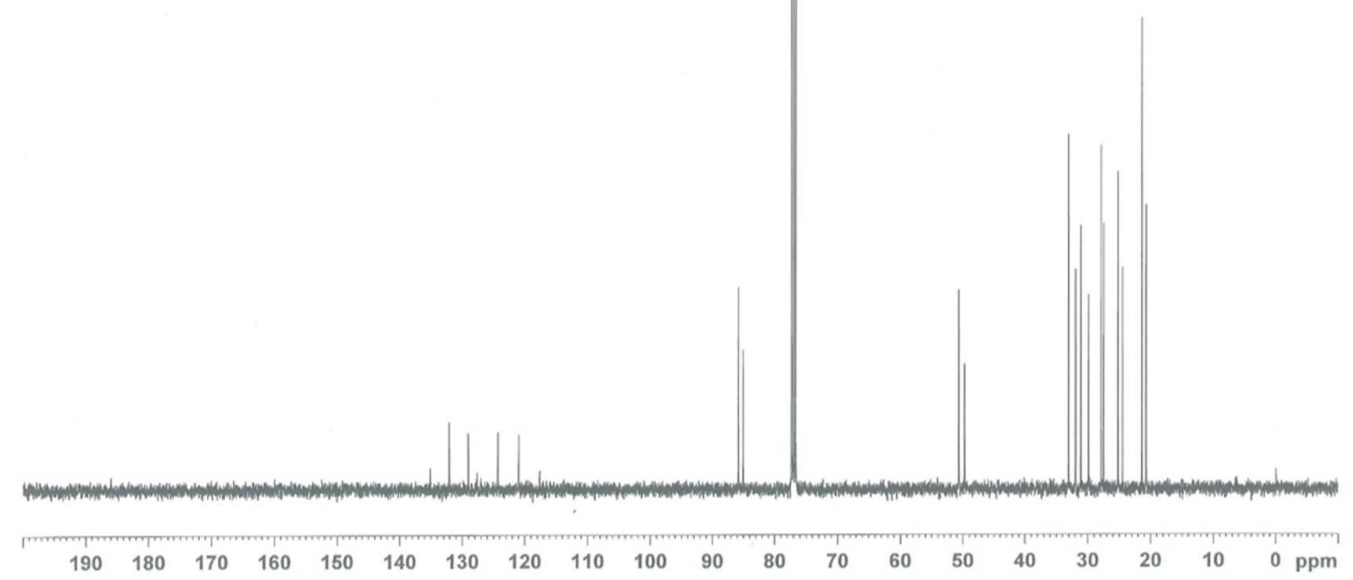




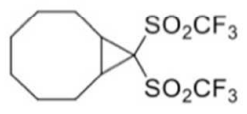

$11 \mathrm{~g}$

$\left({ }^{1} \mathrm{H} \mathrm{NMR}, \mathrm{CDCl}_{3}\right)$
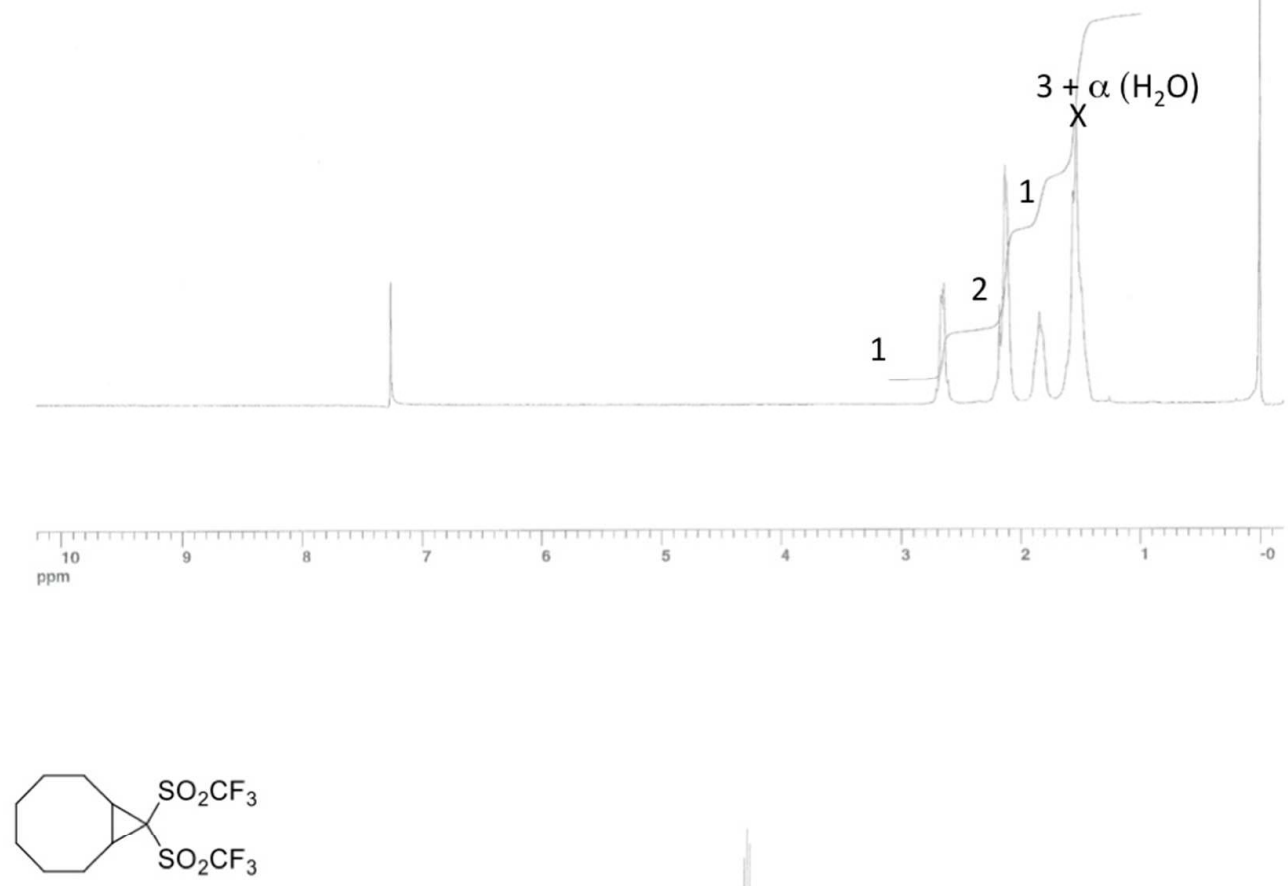

$11 \mathrm{~g}$

$\left({ }^{13} \mathrm{C} \mathrm{NMR}, \mathrm{CDCl}_{3}\right)$

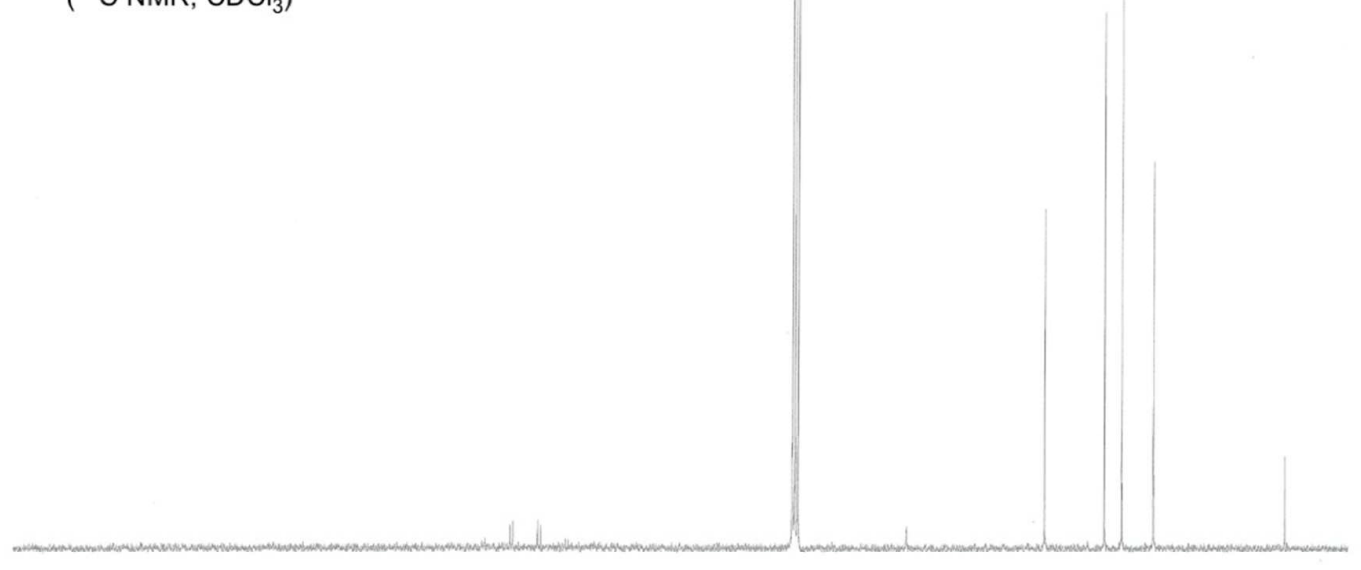

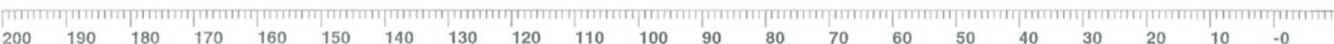
$\begin{array}{llllllllllllllllllllll}200 & 190 & 180 & 170 & 160 & 150 & 140 & 130 & 120 & 110 & 100 & 90 & 80 & 70 & 60 & 50 & 40 & 30 & 20 & 10 & -0\end{array}$ 

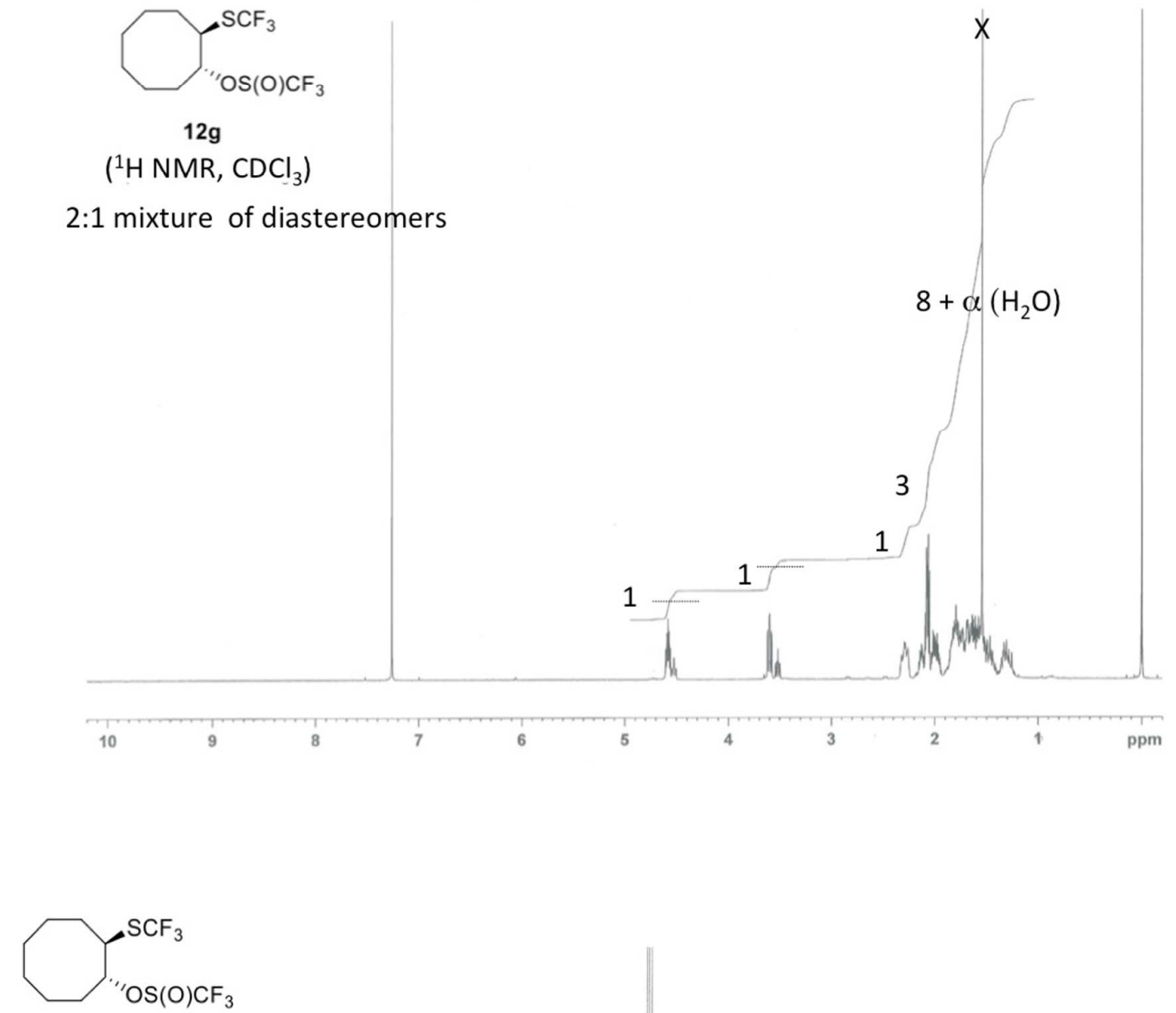

$12 \mathrm{~g}$

$\left({ }^{13} \mathrm{C} \mathrm{NMR}, \mathrm{CDCl}_{3}\right)$
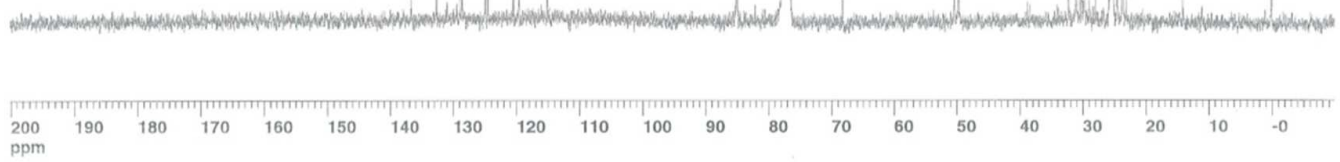


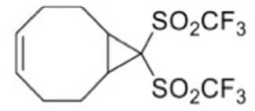

$11 \mathrm{~h}$

$\left({ }^{1} \mathrm{H} \mathrm{NMR}, \mathrm{CDCl}_{3}\right)$

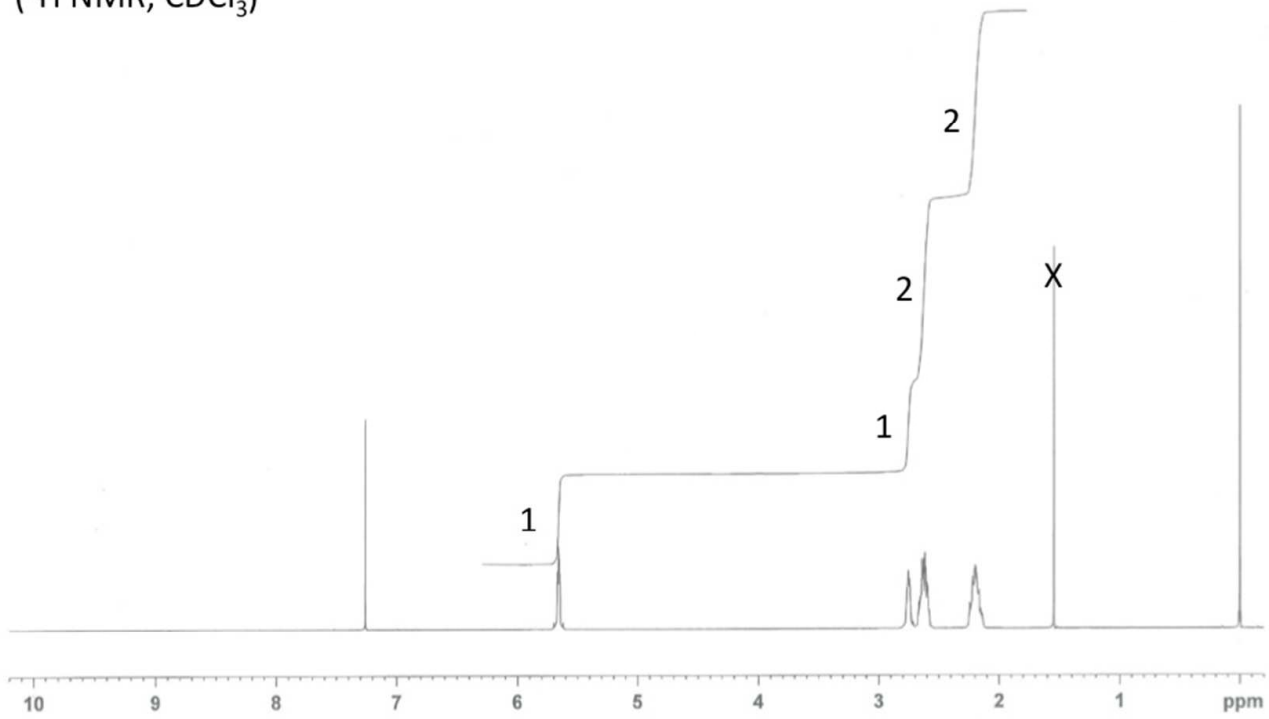

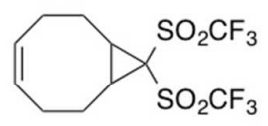

$11 \mathrm{~h}$

$\left({ }^{13} \mathrm{C} \mathrm{NMR} \mathrm{CDCl}_{3}\right)$

${ }^{*}$ contaminated with small amount of $\mathbf{1 2 h}$.

$\mathrm{X}: 12 \mathrm{~h}$

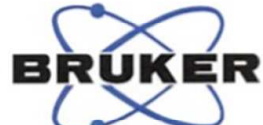
Current Data Parameters
NAME
kmi-COD-CTf2 PROCNO

F2 - Acquisition Parameters Date_r 20160309 TNSTRUM 5.35 PROBHD $5 \mathrm{~mm}$ PABBO BB/ zgpg30
65536

SOLVENT

NS

SWH $\quad 29761.904 \mathrm{~Hz}$

FIDRES $\quad 0.454131 \mathrm{~Hz}$

$\begin{array}{lr}A Q & 1.1010048 \mathrm{sec} \\ \text { RG } & 184.27\end{array}$

16.800 usec $\begin{aligned} 6.50 & \text { use } \\ 298.3 \mathrm{~K} & \end{aligned}$

$.00000000 \mathrm{sec}$

$0.03000000 \mathrm{sec}$

$=== \pm=m$ CHANNEL $f 1 \quad=m=m=m$

SFO1 $125.7703637 \mathrm{C}$

$\begin{array}{ll}\text { P1 } & 13.00 \text { usec } \\ \text { PLW1 } & 90.00000000 \mathrm{w}\end{array}$

=m=m- CHANNEL $f 2$ $2=m=n=z$

SFO2 $\quad 500.1320005 \mathrm{MHz}$

CPDPRG [2 waltz16

PCPD2 100.00 usec

PLW12 $\quad 0.21600001 \mathrm{~W}$

F2 - Processing parameters

$x$

32768
125.7577870
$\mathrm{EM}$

$1.00 \mathrm{~Hz}$

1.40 


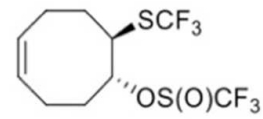

12h

$\left({ }^{1} \mathrm{H} \mathrm{NMR}, \mathrm{CDCl}_{3}\right)$

7:3 mixture of diastereomers
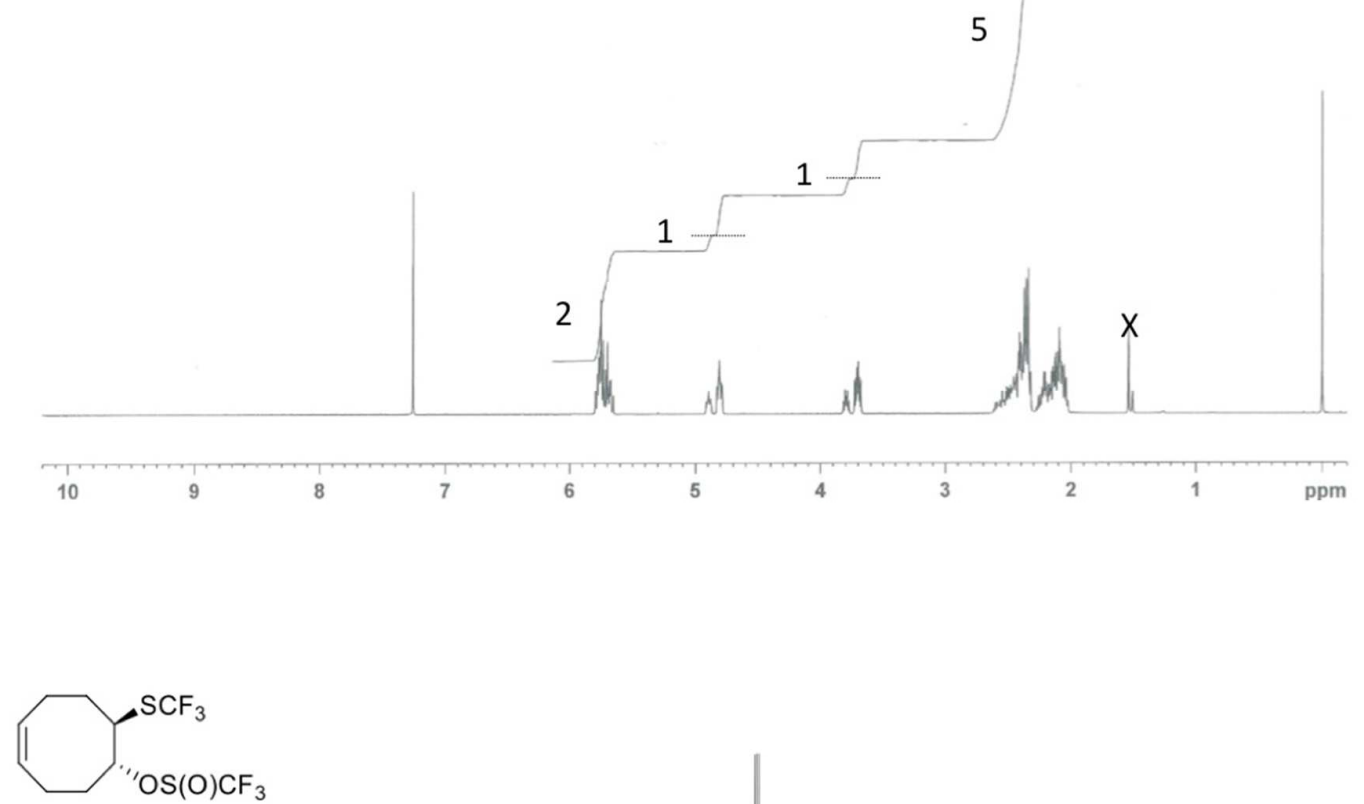

$12 \mathrm{~h}$

$\left({ }^{13} \mathrm{C} \mathrm{NMR}, \mathrm{CDCl}_{3}\right)$ 


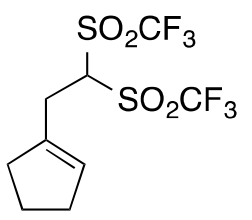

13

( ${ }^{1} \mathrm{H}$ NMR, $\mathrm{CDCl}_{3}$ )
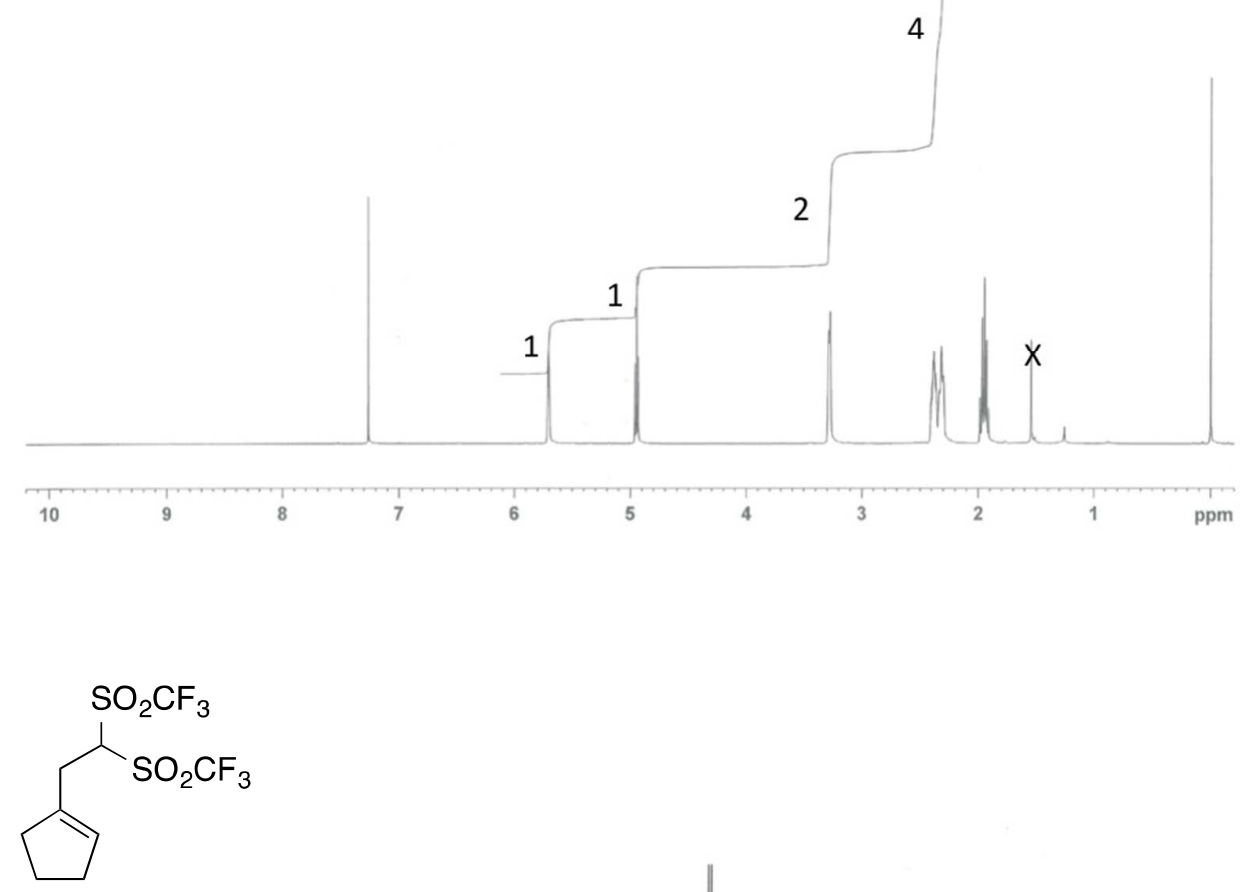

13

$\left({ }^{13} \mathrm{C} \mathrm{NMR}, \mathrm{CDCl}_{3}\right)$

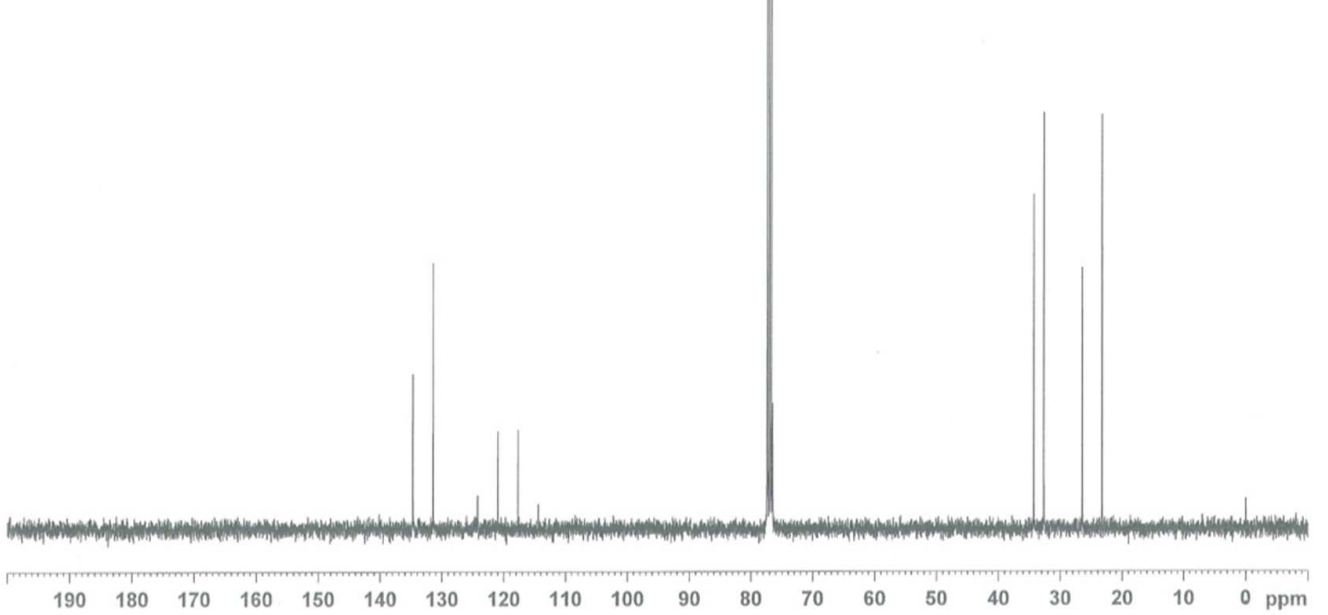




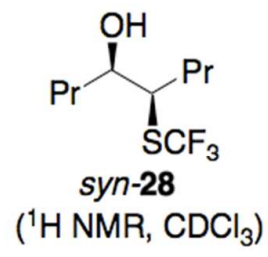

$\left({ }^{1} \mathrm{H} \mathrm{NMR}, \mathrm{CDCl}_{3}\right)$
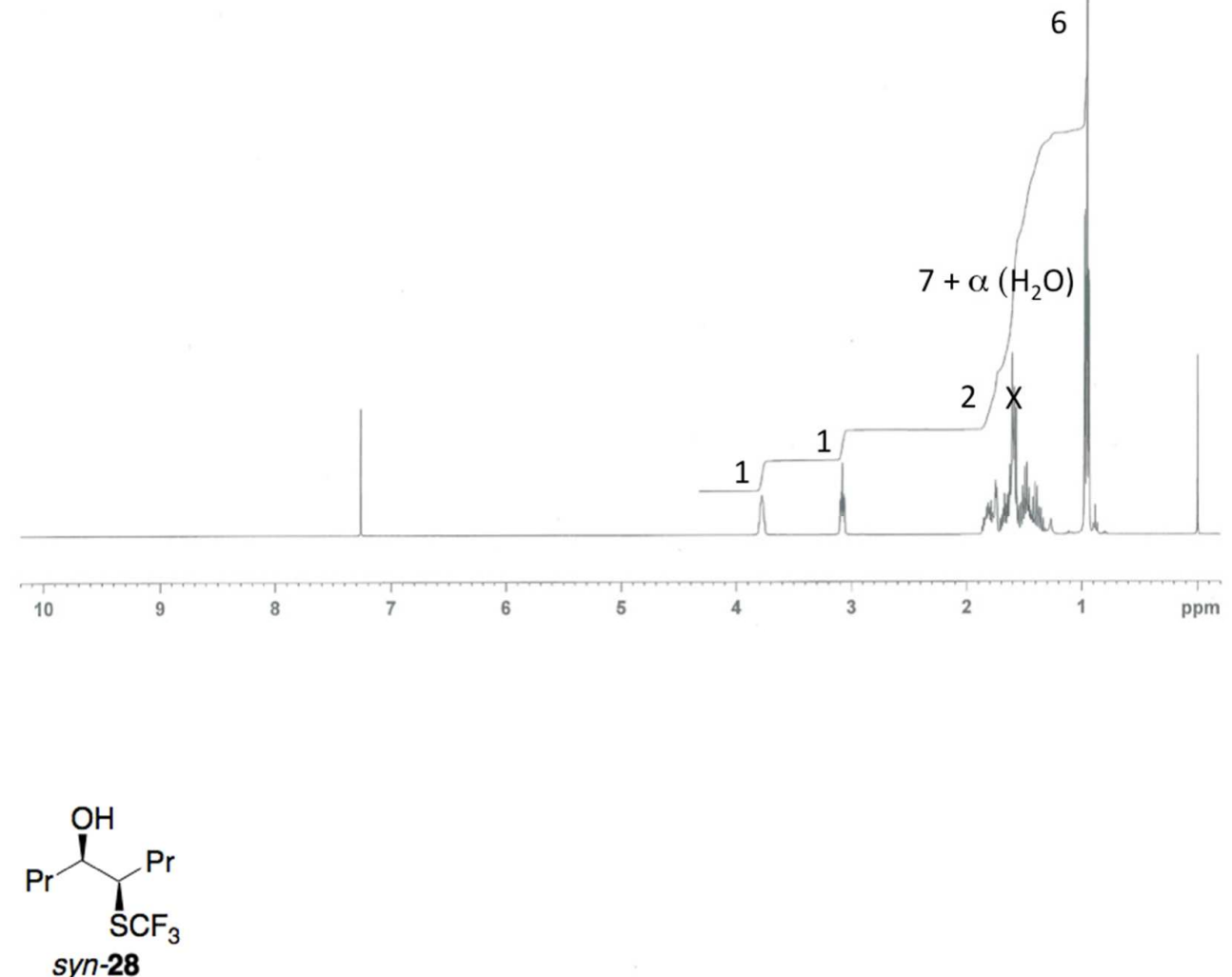

$\left({ }^{13} \mathrm{C} \mathrm{NMR}, \mathrm{CDCl}_{3}\right)$

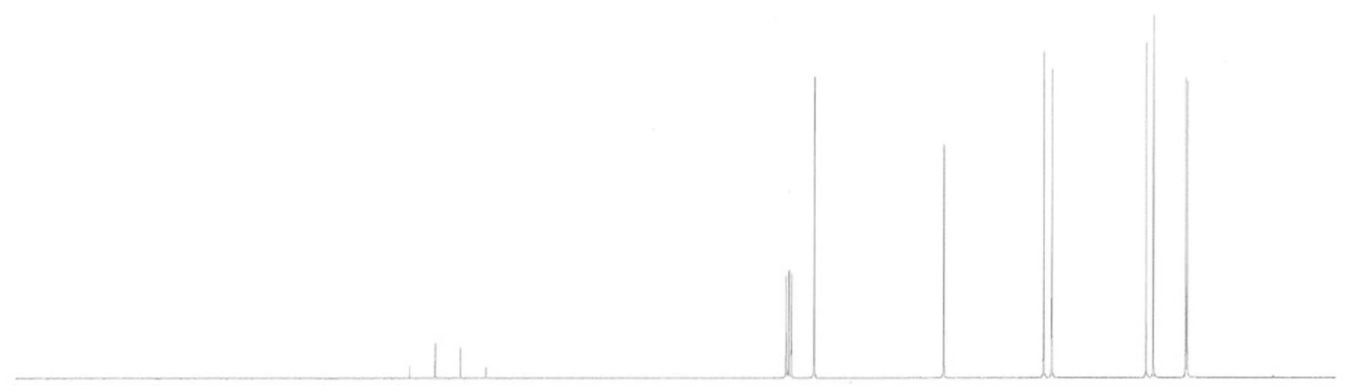

$200 \quad 190 \quad 180 \quad 170 \quad 160 \quad 150 \quad 140 \quad 130 \quad 120 \quad 110 \quad 100 \quad 90-80 \quad 70 \quad 60-50 \quad 40 \quad 3020$ 

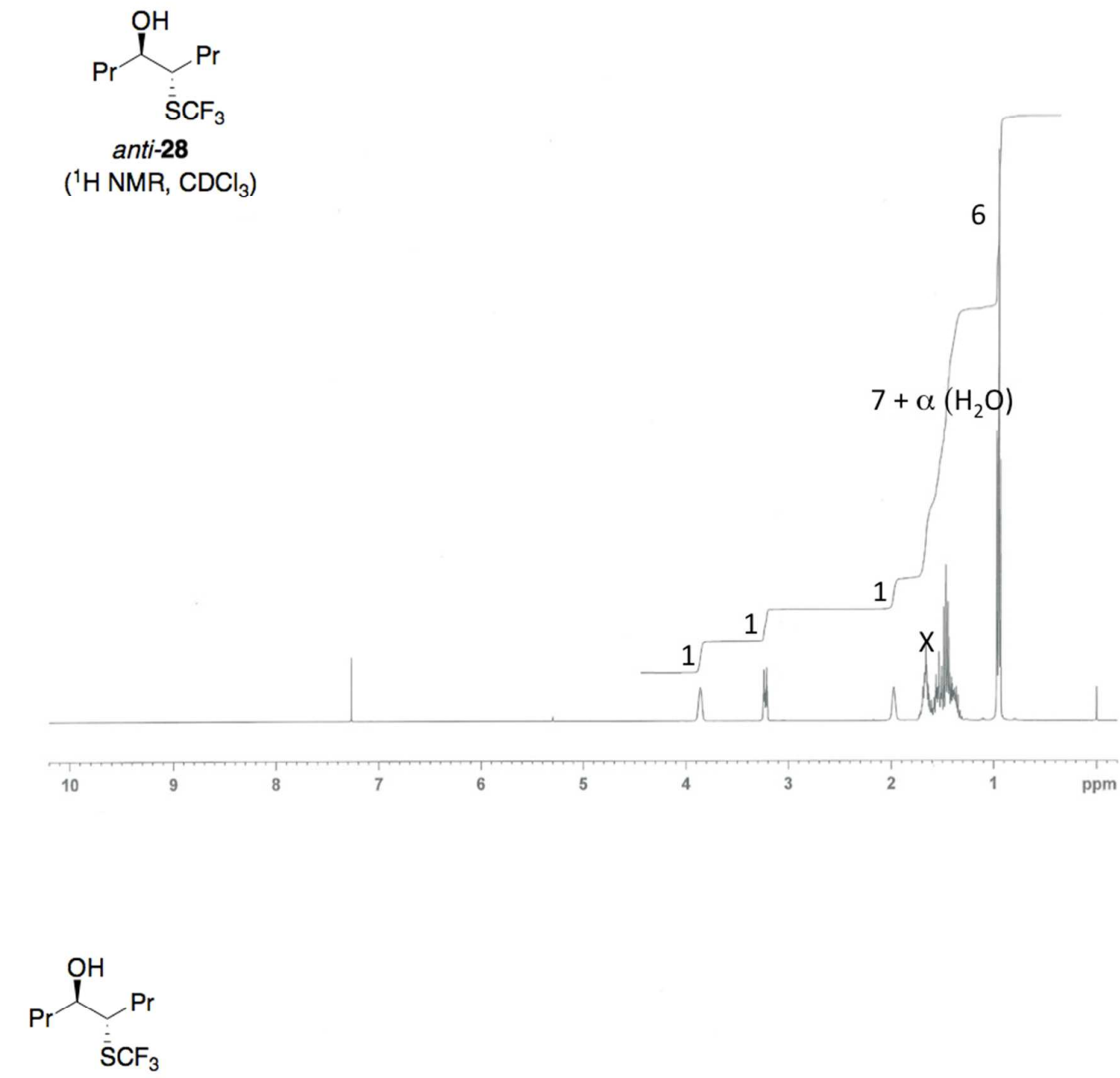

anti-28

$\left({ }^{13} \mathrm{C} \mathrm{NMR}, \mathrm{CDCl}_{3}\right)$

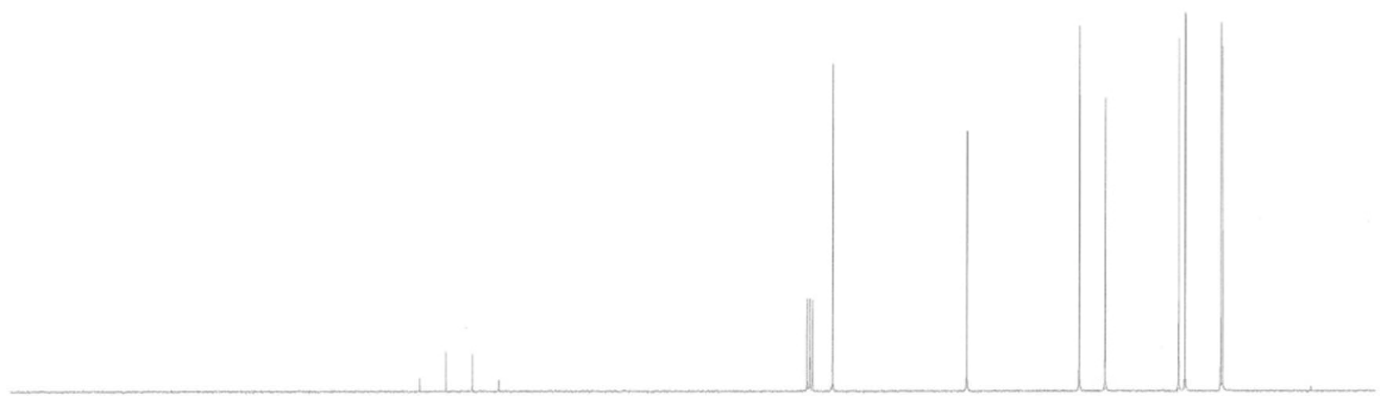

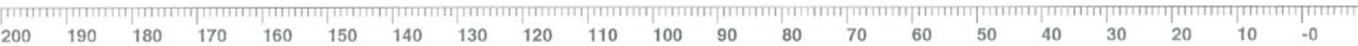

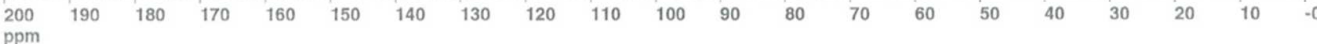



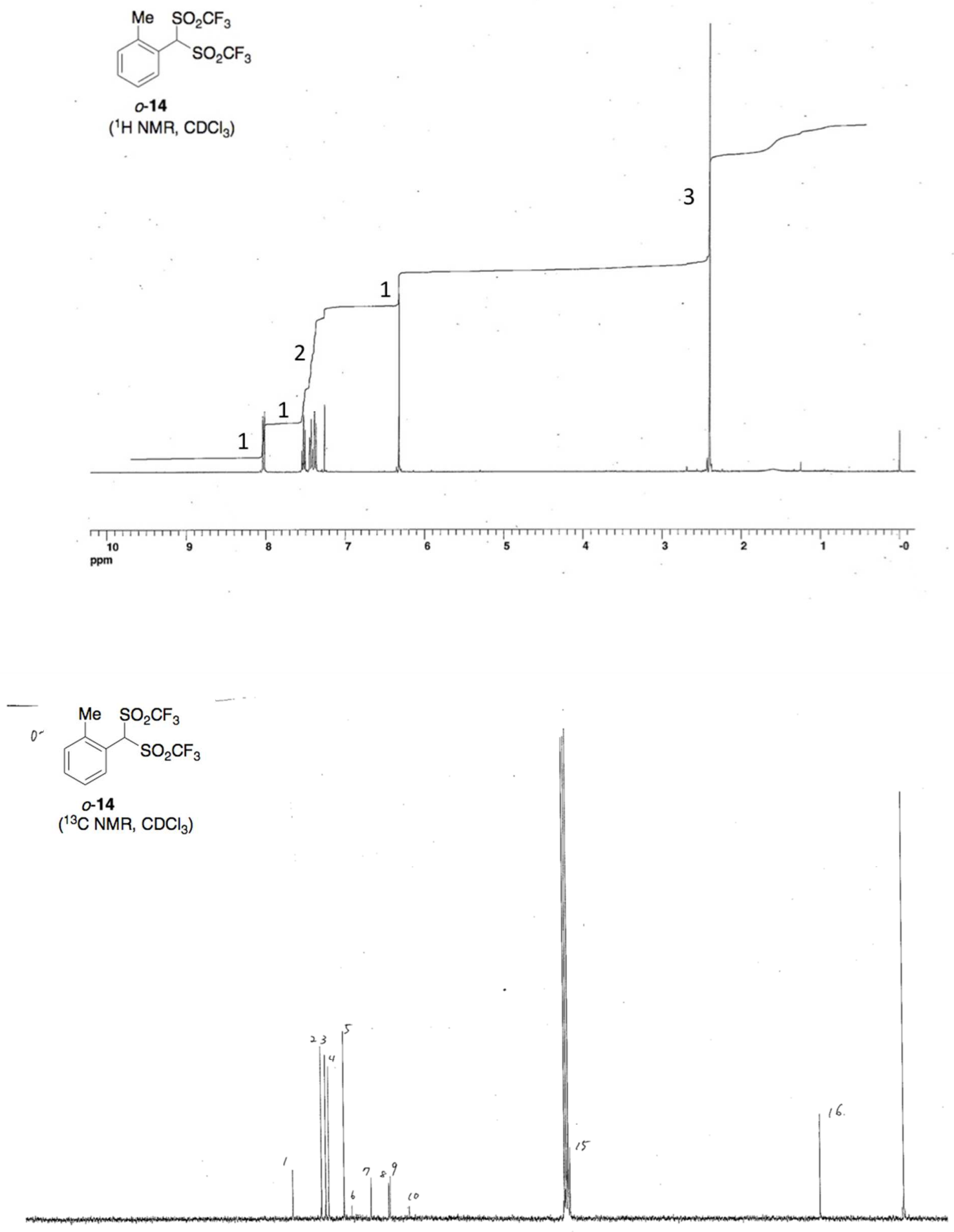

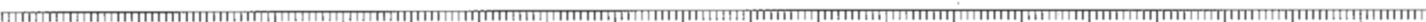
$\begin{array}{lllllllllllllllllllll}200 & 190 & 180 & 170 & 160 & 150 & 140 & 130 & 120 & 110 & 100 & 90 & 80 & 70 & 60 & 50 & 40 & 30 & 20 & 10 & -0\end{array}$ 

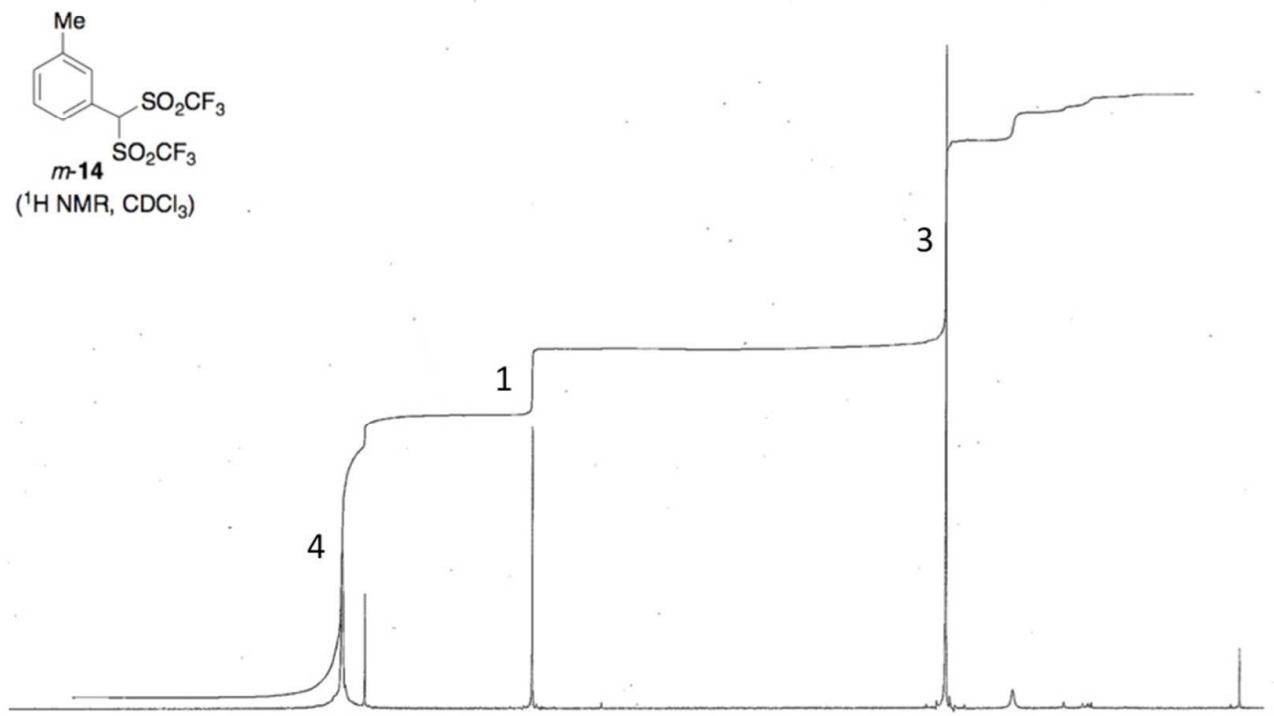

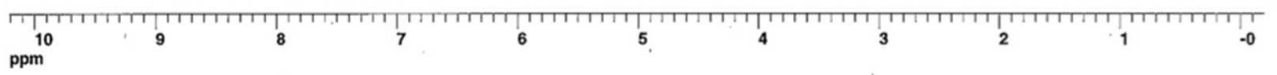

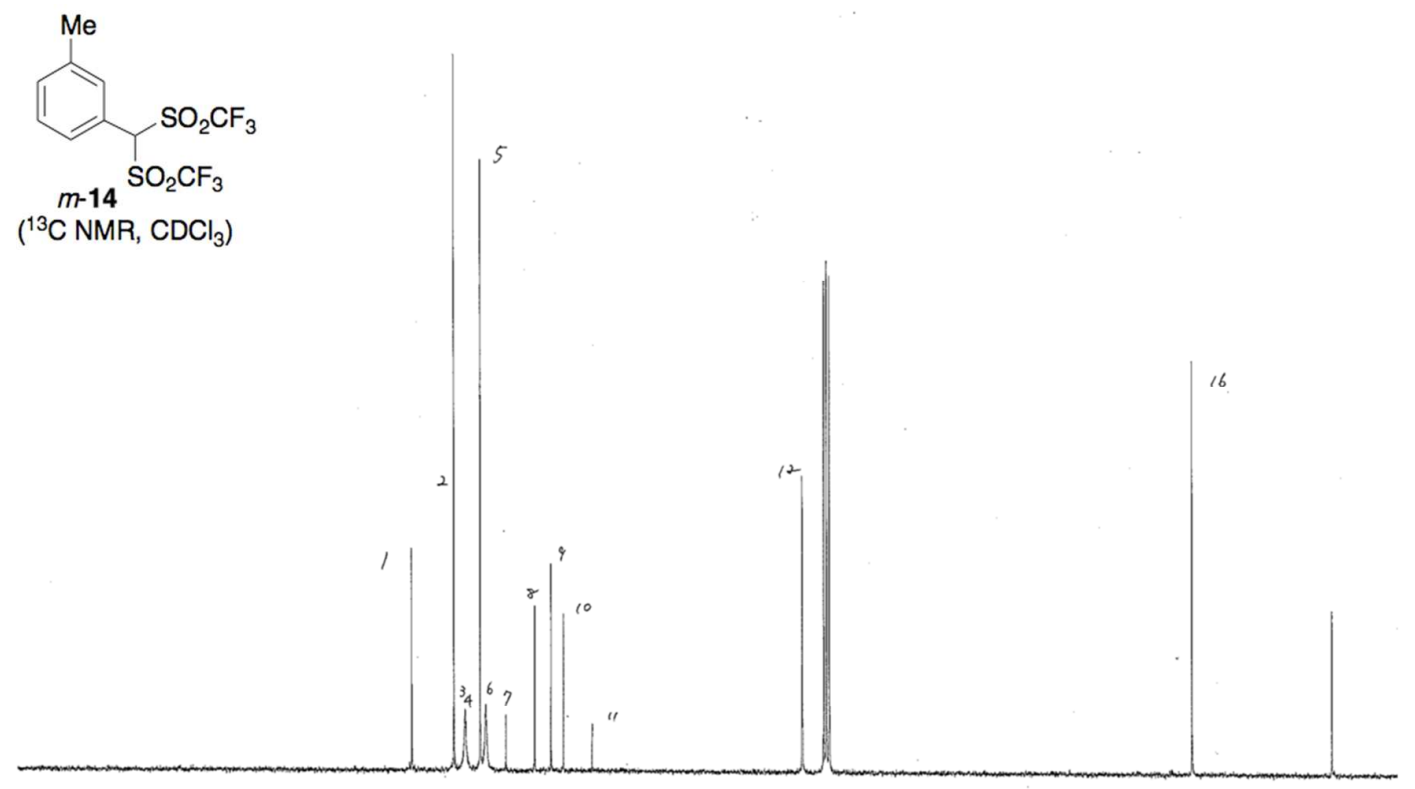

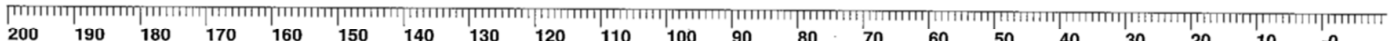
ppm

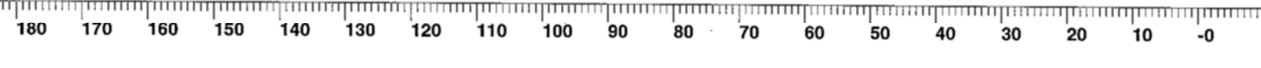




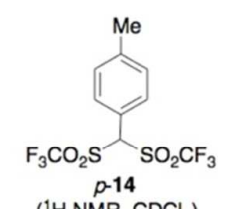

$\left({ }^{1} \mathrm{H} \mathrm{NMR}, \mathrm{CDCl}_{3}\right)$
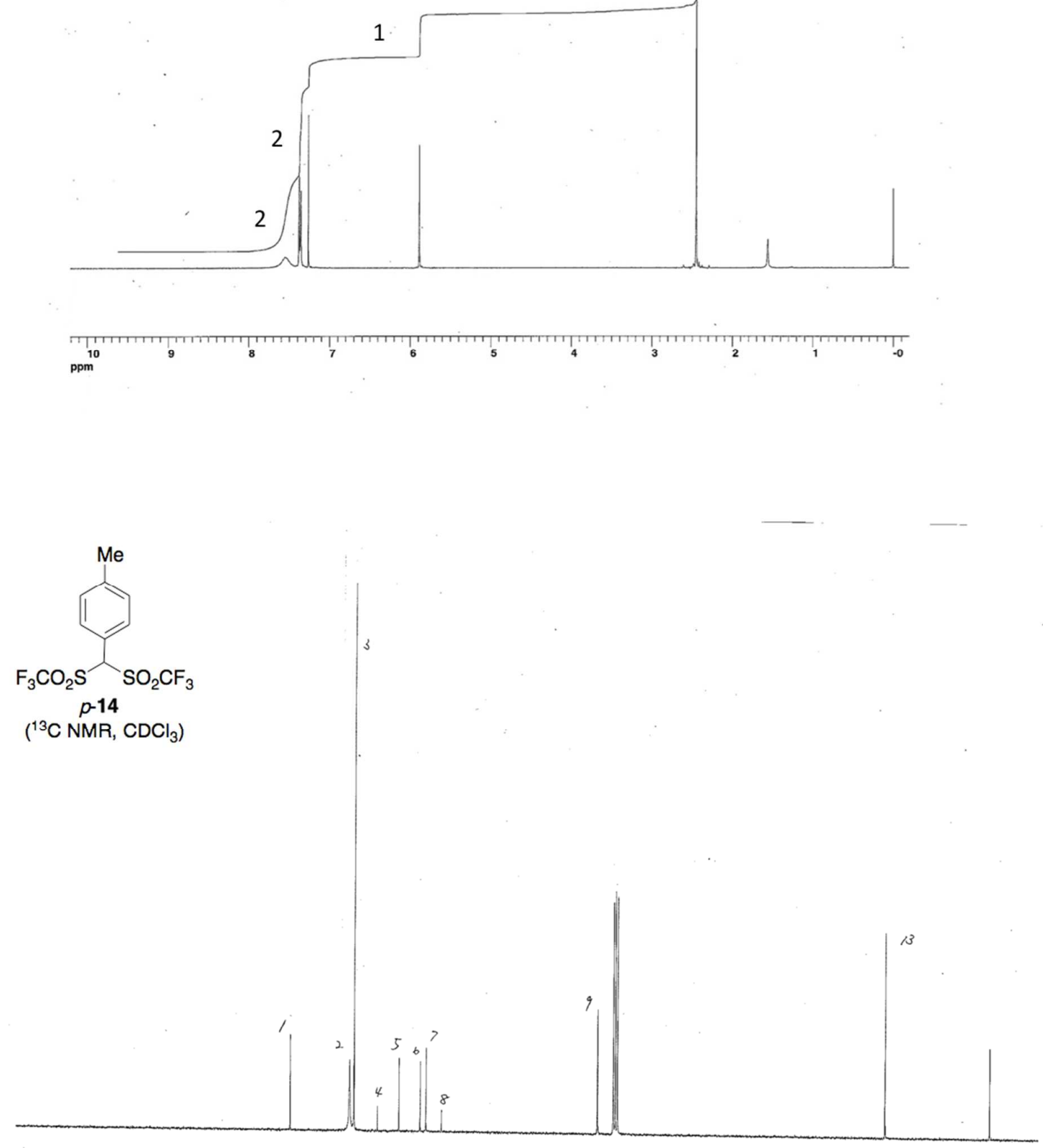

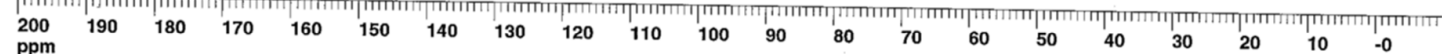



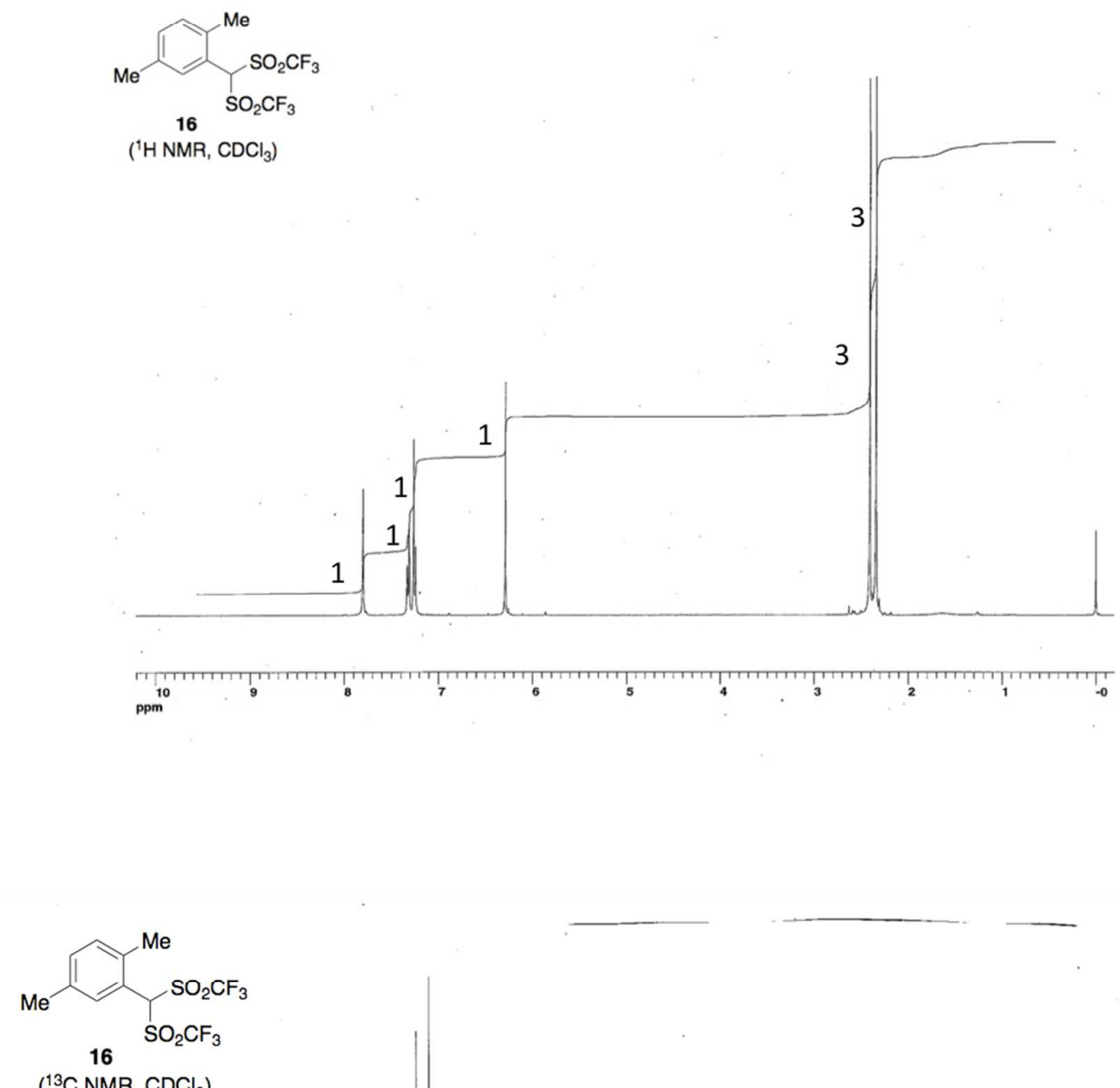

$\left({ }^{13} \mathrm{C} \mathrm{NMR}, \mathrm{CDCl}_{3}\right.$ )

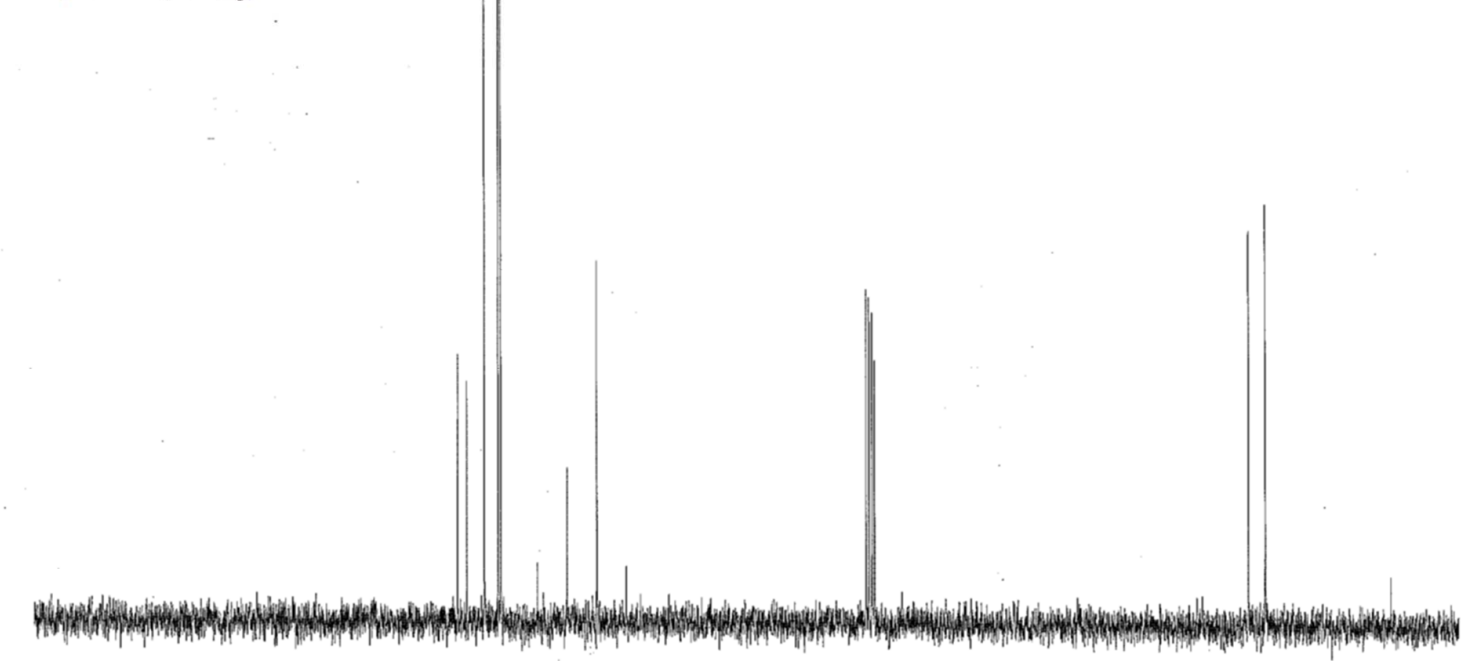

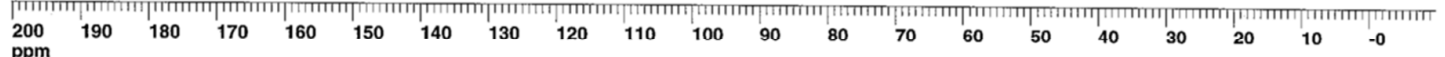

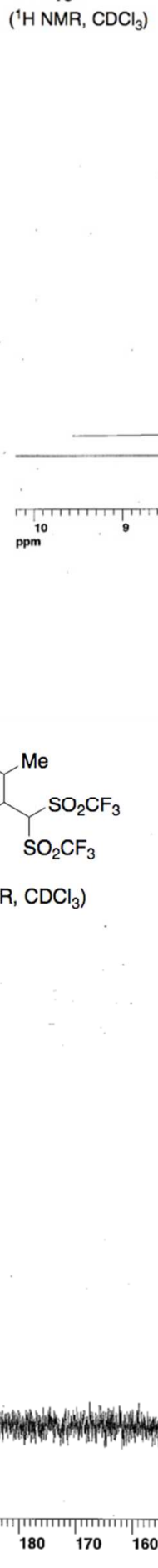

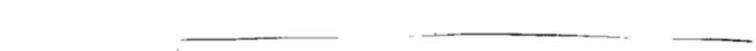




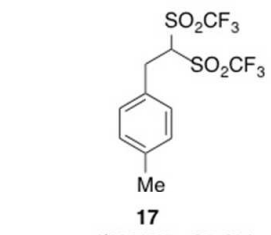

( ${ }^{1} \mathrm{H} \mathrm{NMR,} \mathrm{CDCl}_{3}$ )
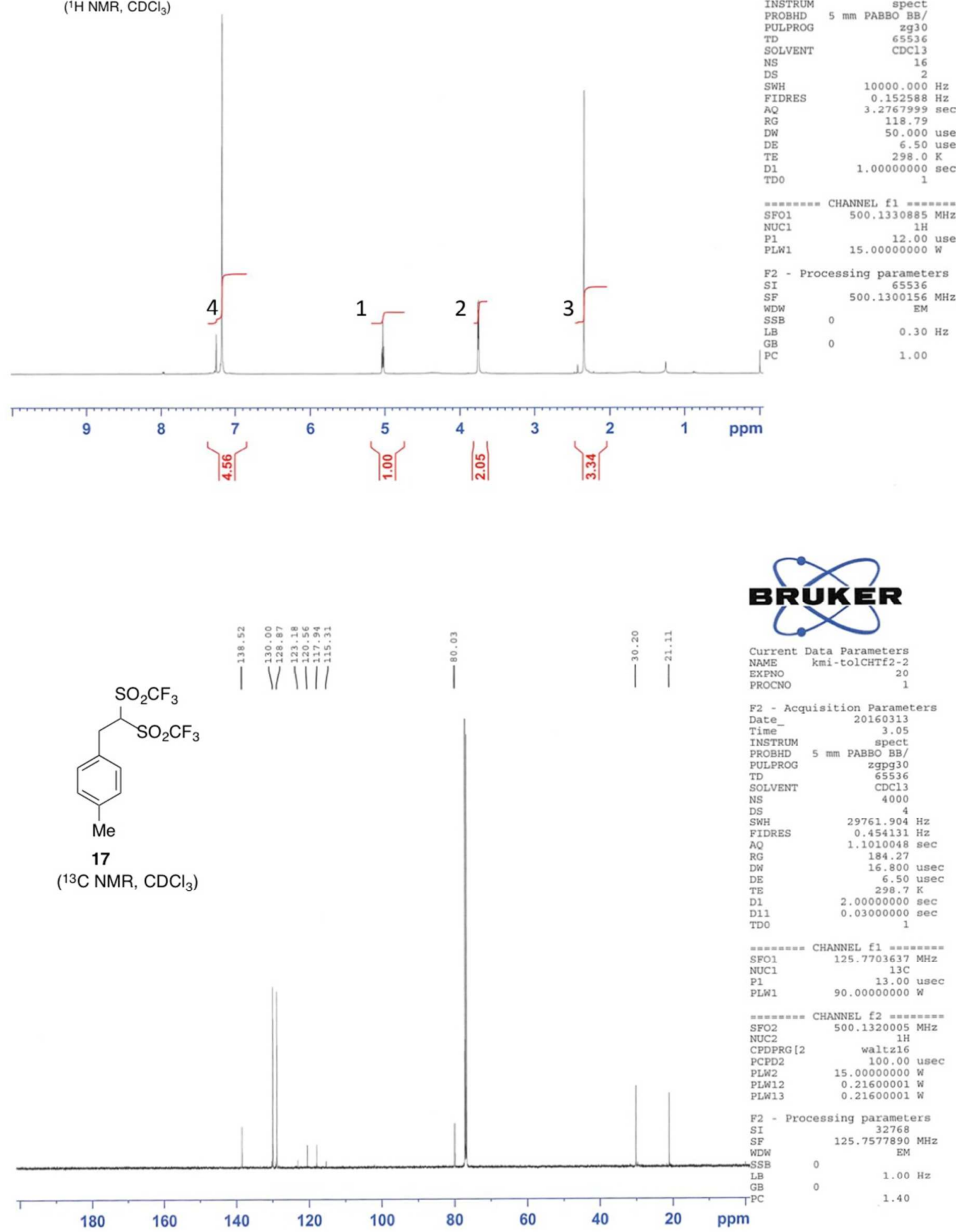

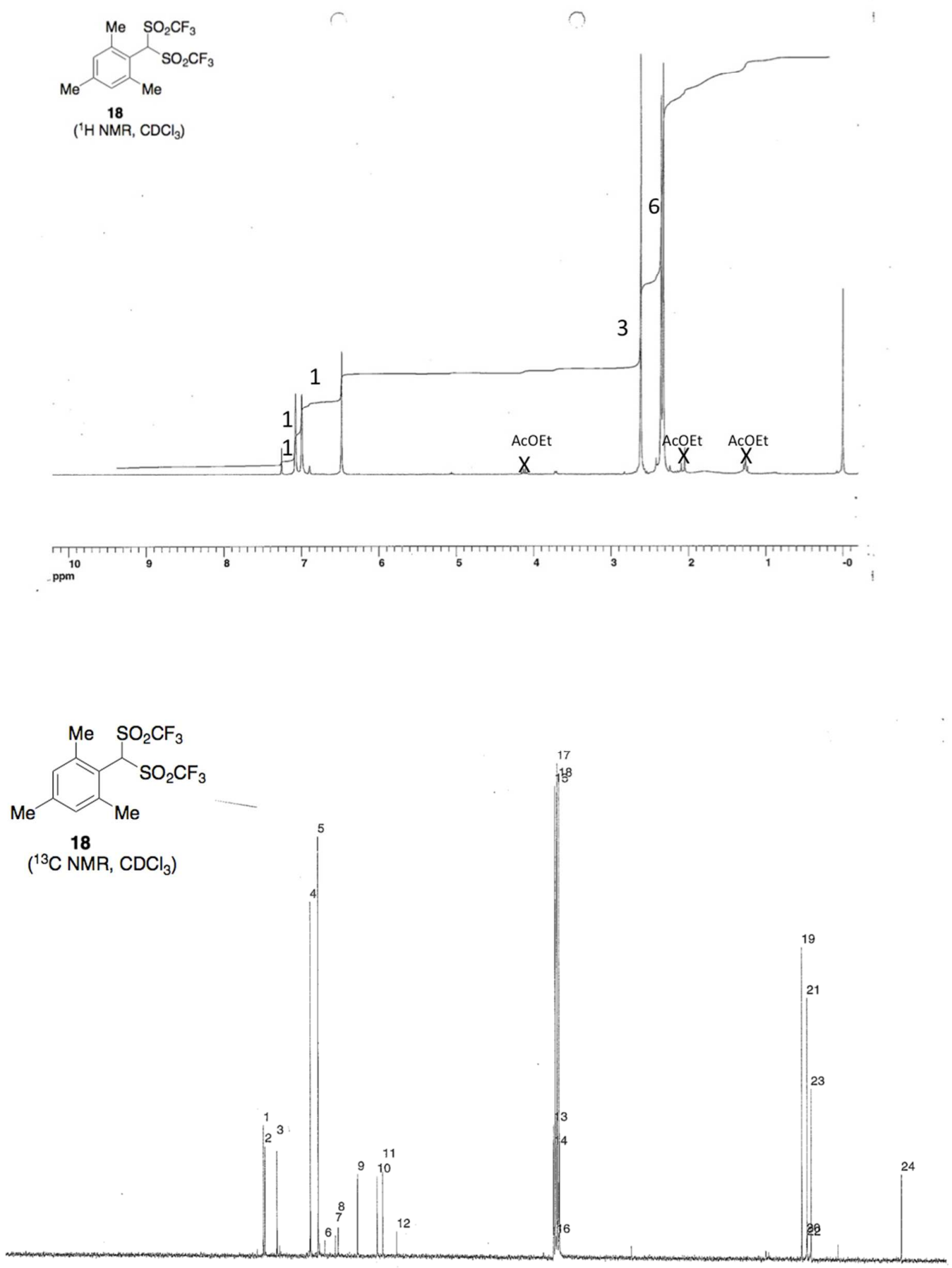

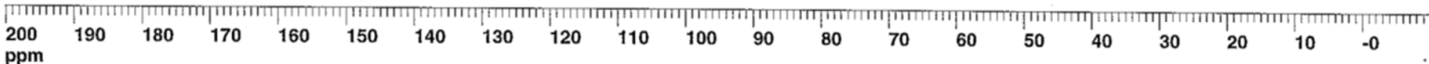
ppm 

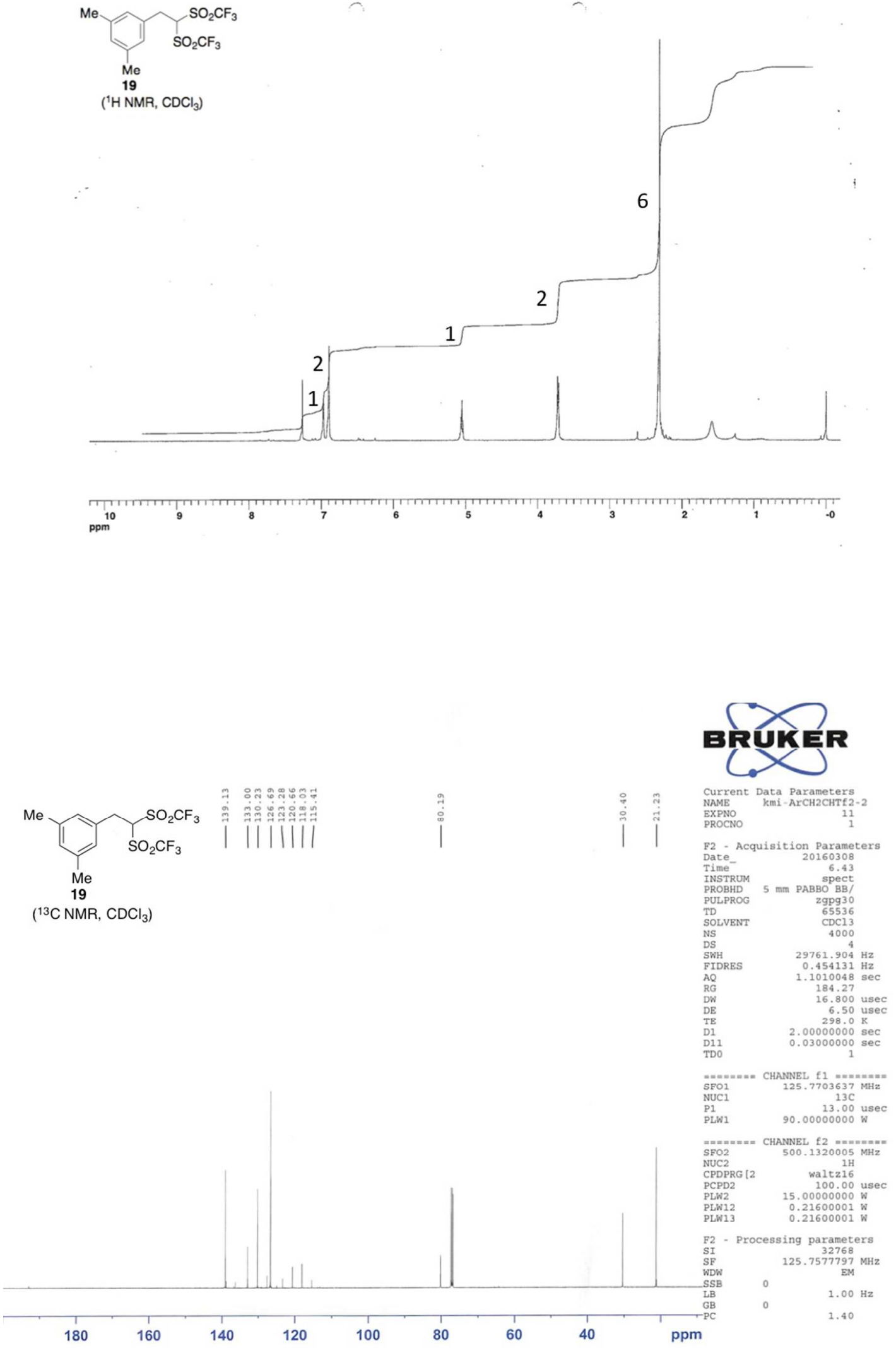

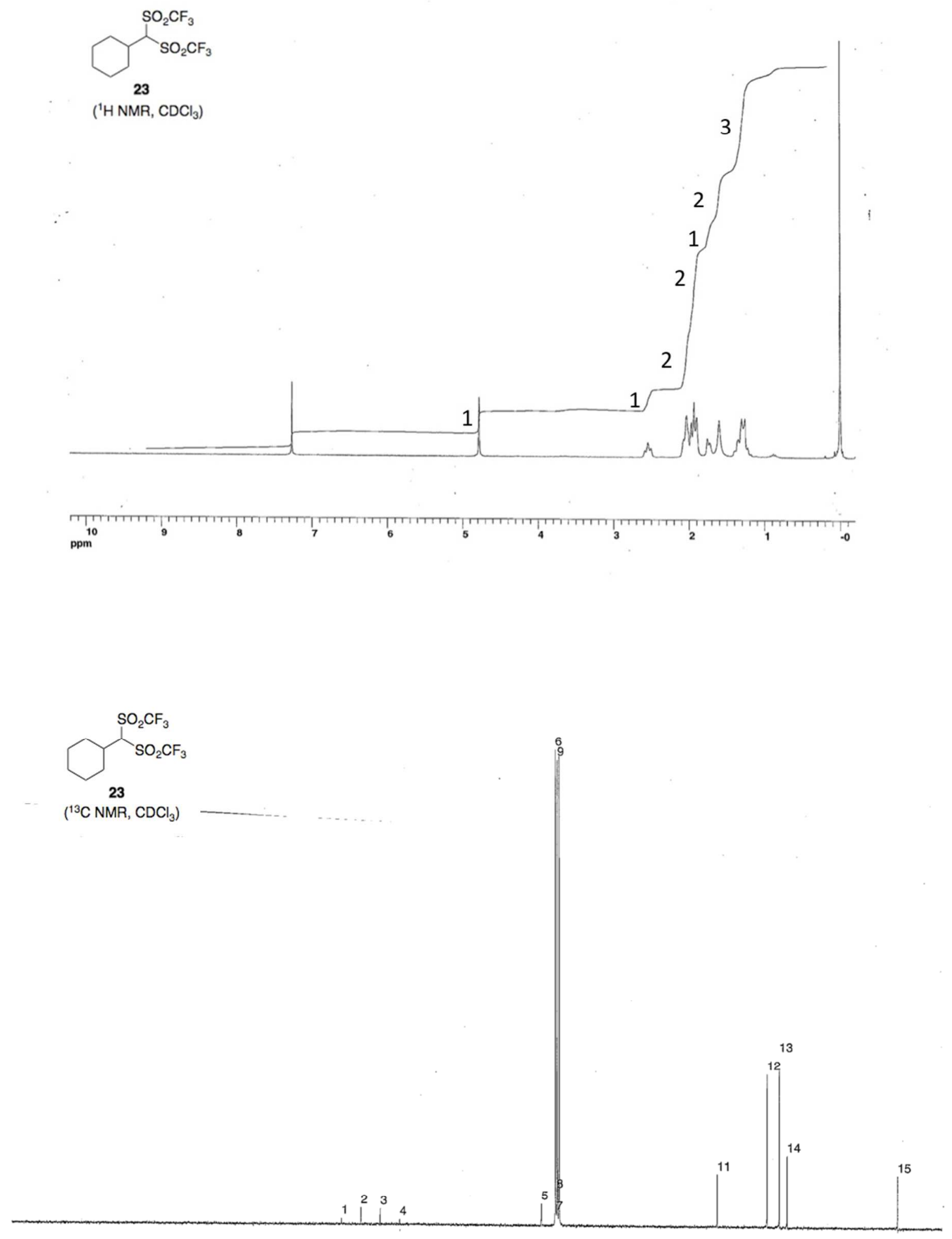

$\begin{array}{lllllllllllllllllllll}200 & 190 & 180 & 170 & 160 & 150 & 140 & 130 & 120 & 110 & 100 & 90 & 80 & 70 & 60 & 50 & 40 & 30 & 20 & 10 & -0\end{array}$ 200 


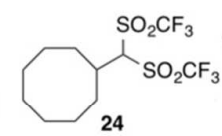

$\left({ }^{1} \mathrm{H} \mathrm{NMR}, \mathrm{CDCl}_{3}\right)$
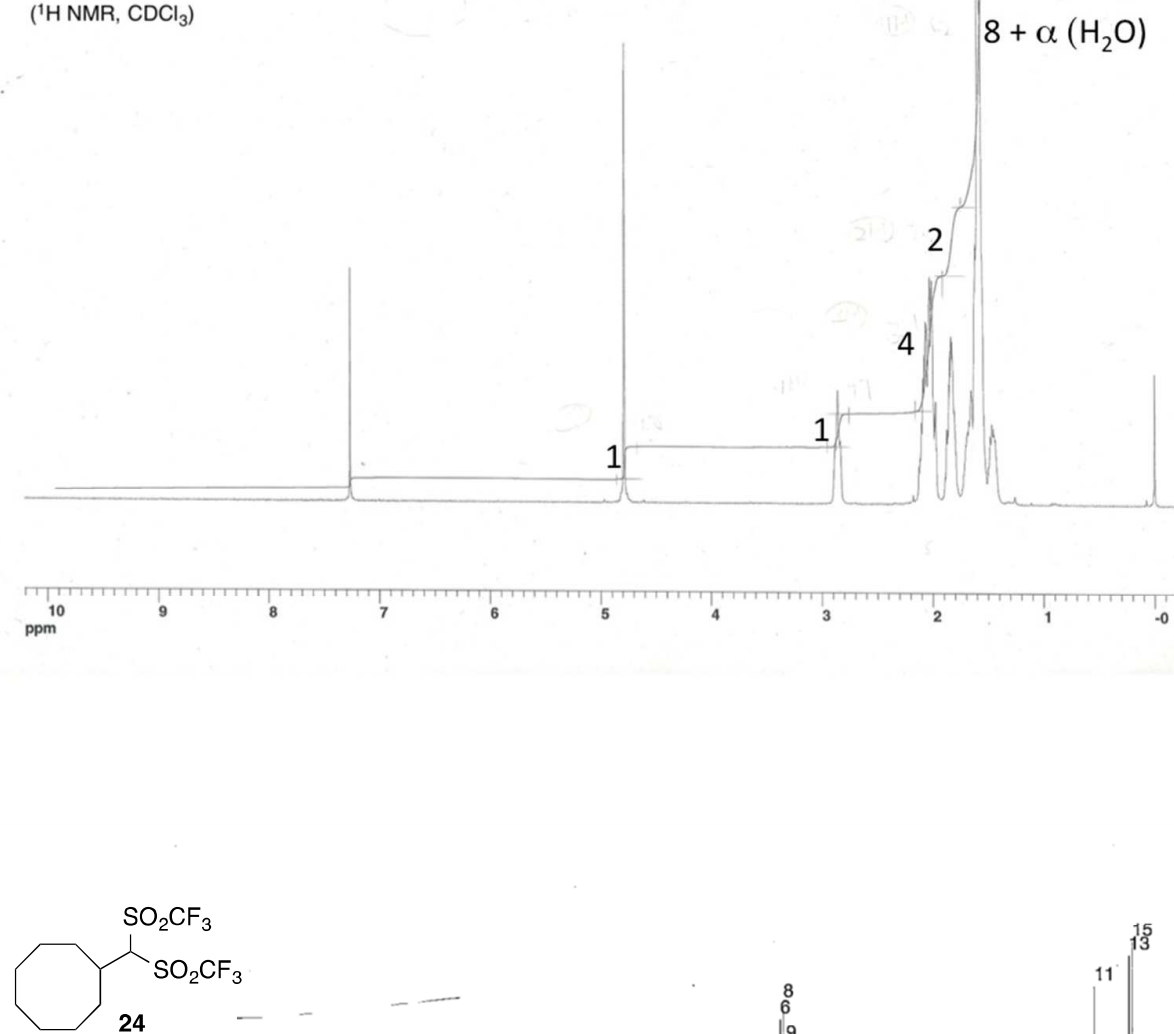

$\left({ }^{1} \mathrm{H} \mathrm{NMR}, \mathrm{CDCl}_{3}\right)$

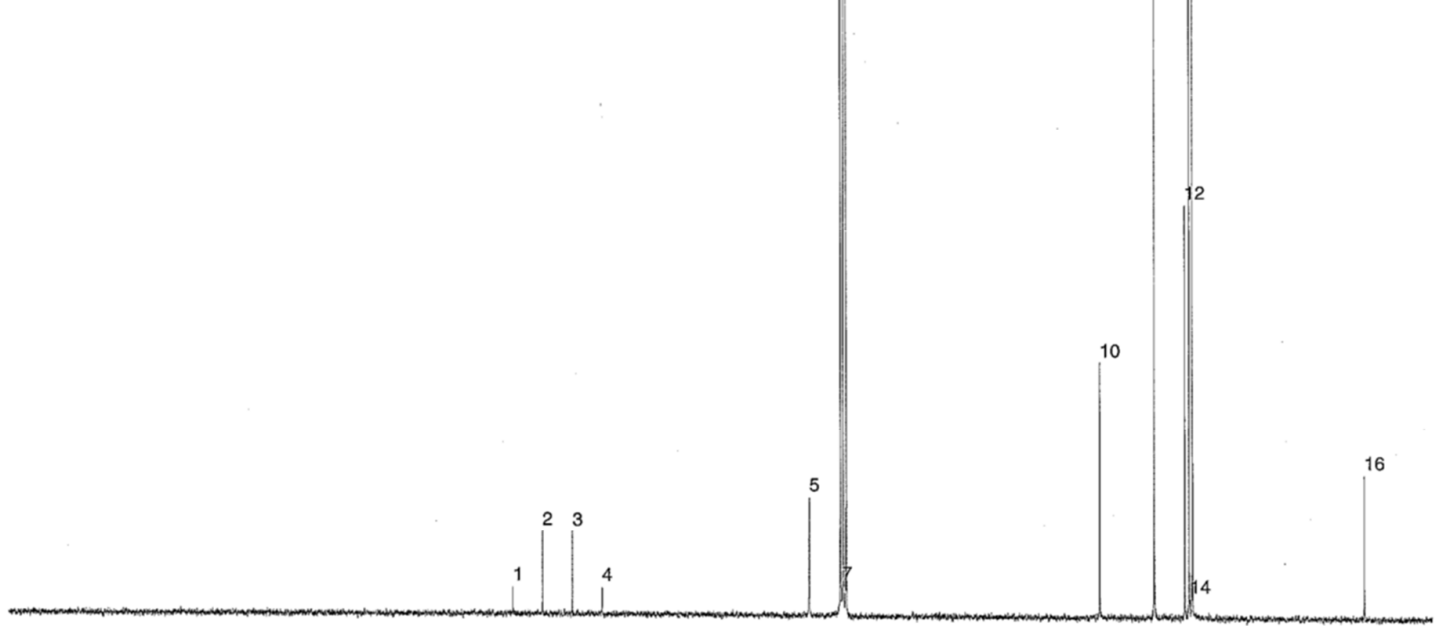

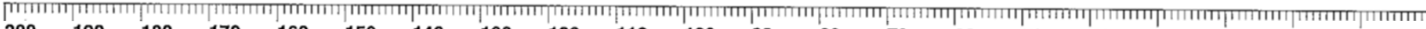
$\begin{array}{lllllllllllllllllllll}200 & 190 & 180 & 170 & 160 & 150 & 140 & 130 & 120 & 110 & 100 & 90 & 80 & 70 & 60 & 50 & 40 & 30 & 20 & 10 & -0\end{array}$ 

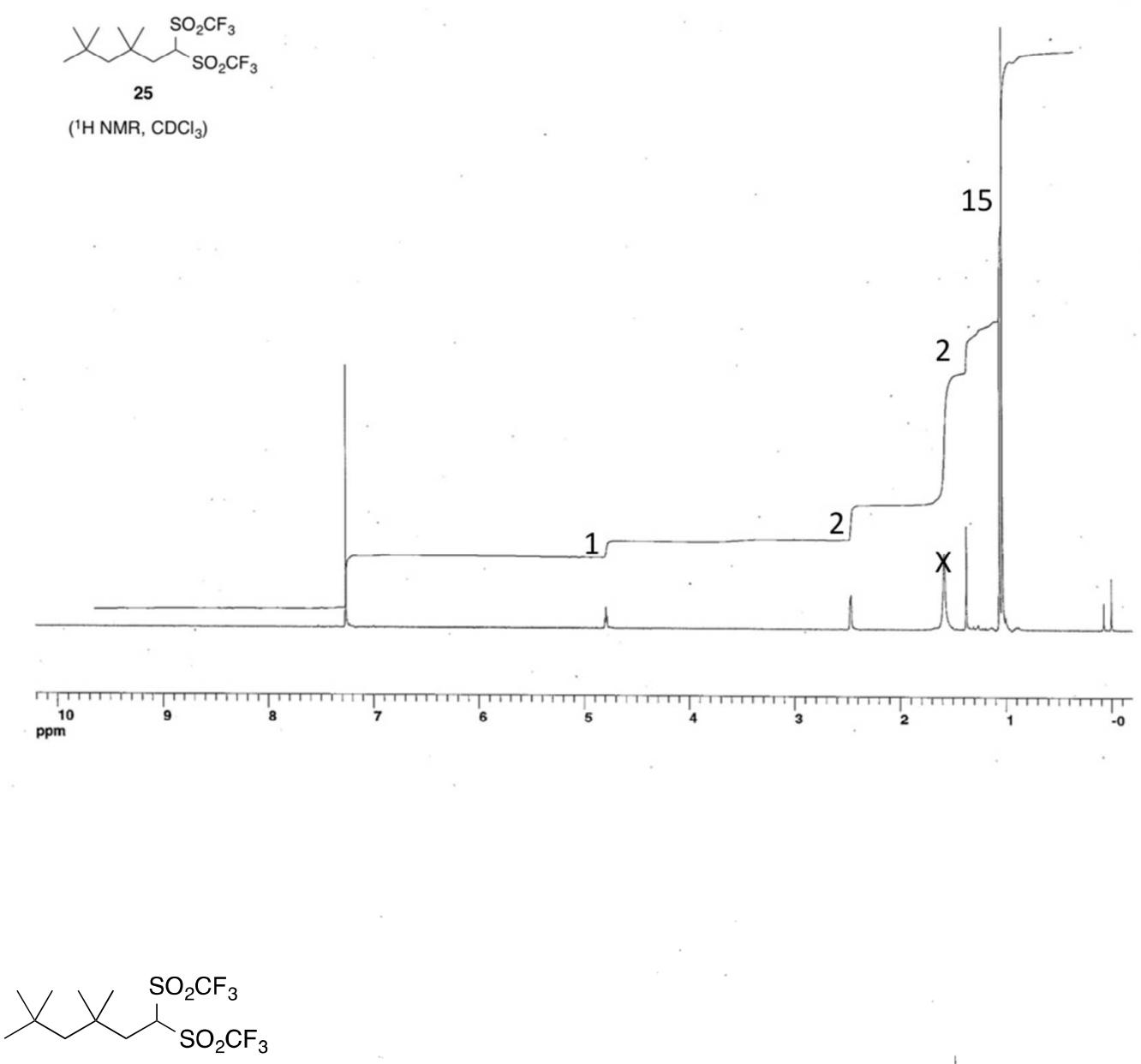

25

$\left({ }^{13} \mathrm{C} \mathrm{NMR}, \mathrm{CDCl}_{3}\right)$

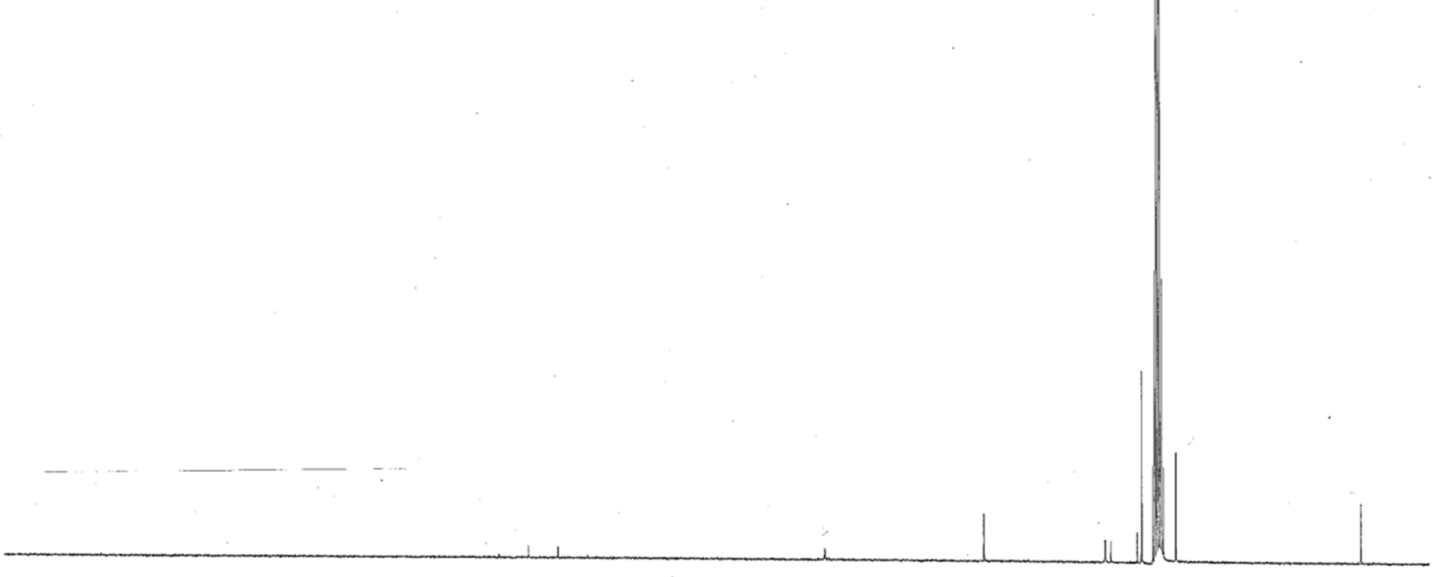

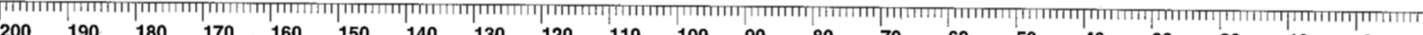

$\begin{array}{lllllllllllllllllllllll}200 & 190 & 180 & 170 & 160 & 150 & 140 & 130 & 120 & 110 & 100 & 90 & 80 & 70 & 60 & 50 & 40 & 30 & 20 & 10 & -0\end{array}$ 


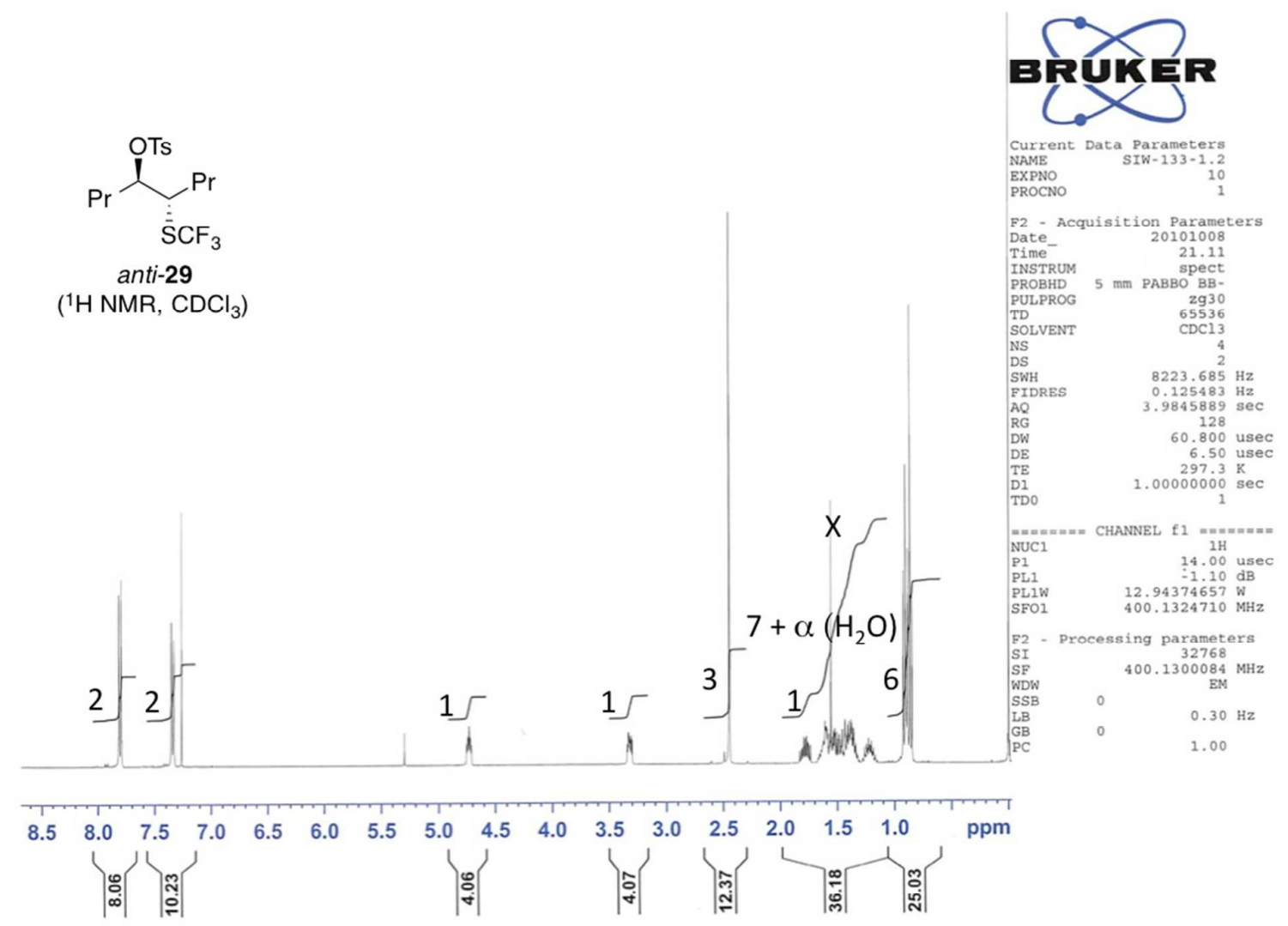

